\title{
Production and Characterization of a Detector Package for the Xenon Electroluminescent Prototype
}

\author{
by \\ Matthew G. Dunford \\ A Thesis submitted to \\ the Faculty of Graduate and Postdoctoral Affairs \\ in partial fulfilment of \\ the requirements for the degree of \\ Master of Science \\ Ottawa-Carleton Institute for \\ Physics \\ Department of Physics \\ Carleton University \\ Ottawa, Ontario, Canada
}

July 23,2012

Copyright (C)

2012 - Matthew G. Dunford 
Library and Archives

Canada

Published Heritage

Branch

395 Wellington Street

Ottawa ON K1A ON4

Canada
Bibliothèque et

Archives Canada

Direction du

Patrimoine de l'édition

395 , rue Wellington

Ottawa ON K1A ON4

Canada
Your file Votre référence

ISBN: 978-0-494-93633-7

Our file Notre référence

ISBN: $978-0-494-93633-7$
NOTICE:

The author has granted a nonexclusive license allowing Library and Archives Canada to reproduce, publish, archive, preserve, conserve, communicate to the public by telecommunication or on the Internet, loan, distrbute and sell theses worldwide, for commercial or noncommercial purposes, in microform, paper, electronic and/or any other formats.

The author retains copyright ownership and moral rights in this thesis. Neither the thesis nor substantial extracts from it may be printed or otherwise reproduced without the author's permission.
AVIS:

L'auteur a accordé une licence non exclusive permettant à la Bibliothèque et Archives Canada de reproduire, publier, archiver, sauvegarder, conserver, transmettre au public par télécommunication ou par l'Internet, prêter, distribuer et vendre des thèses partout dans le monde, à des fins commerciales ou autres, sur support microforme, papier, électronique et/ou autres formats.

L'auteur conserve la propriété du droit d'auteur et des droits moraux qui protege cette thèse. $\mathrm{Ni}$ la thèse ni des extraits substantiels de celle-ci ne doivent être imprimés ou autrement reproduits sans son autorisation.
In compliance with the Canadian Privacy Act some supporting forms may have been removed from this thesis.

While these forms may be included in the document page count, their removal does not represent any loss of content from the thesis.
Conformément à la loi canadienne sur la protection de la vie privée, quelques formulaires secondaires ont été enlevés de cette thèse.

Bien que ces formulaires aient inclus dans la pagination, il n'y aura aucun contenu manquant. 


\section{Abstract}

In order to probe the properties of neutrinos, a prototype time projection chamber is under construction at Carleton. This prototype is known as the Xenon Electroluminescent Prototype (XEP) and will endeavour to demonstrate $<1 \%$ energy resolution and good background rejection through precise spatial resolution.

An energy resolution of $1.75 \%$ at a maximum gain of 20,000 is shown for XEP's first detector package. The production of a CsI photocathode by vacuum evaporation shows thickness variations of $\sim 10 \mathrm{~nm}$, corresponding to a $<1 \%$ variation in quantum efficiency. The data acquisition electronics to be used with XEP are shown to have roughly $2 \mathrm{mV}$ of noise per channel, which will not deteriorate XEP's energy resolution for sufficiently large pulses. 
I dedicate this thesis to itself. 


\section{Acknowledgments}

First and foremost I would like to thank (Mr.) Chris Warren. It took me a few years, but I finally realized that physics is what I should be doing.

Next I must thank Dr. Kevin Graham. Your guidance over the last few years of my academic career has been invaluable. You have shown me that experimental physics can often be challenging, but is also rewarding. Thank you.

I must also thank the many people whom I have worked with on XEP: Dr. Chris Ouellet, Dr. Etienne Rollin, Dr. Cliff Hargrove, Dr. David Sinclair, Philippe Gravelle, Yves Baribeau, Matt Bowcock, Francois Leonard, Jillian Henderson, Maria Rozo, Ryan Killick, and Roozbeh Mody; you have all made my time working on XEP an enjoyable one. 


\section{Table of Contents}

Abstract $\quad$ ii

Acknowledgments iv

Table of Contents v v

List of Tables viii

List of Figures $\quad$ ix

1 Introduction 1

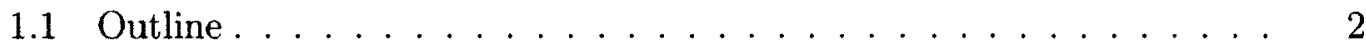

1.2 Author's Contribution .................. 3

2 Theory 4

2.1 Neutrino physics . . . . . . . . . . . . . . . . 4

2.1 .1 The neutrino ..................... 4

2.1 .2 Neutrino oscillations . . . . . . . . . . . 7

2.1 .3 Neutrino mass . . . . . . . . . . . . . . . 10

2.2 Double beta decay . . . . . . . . . . . . . . . . . . 13

2.2 .1 Electron energy spectrum . . . . . . . . . . . 16

2.2 .2 Double beta decay of ${ }^{136} \mathrm{Xe} \ldots \ldots \ldots 17$ 
3 Signal Generation and Detection in XEP 20

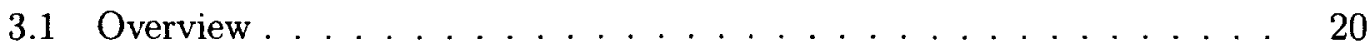

3.2 Ionization and scintillation . . . . . . . . . . . . . . 21

3.3 Cesium iodide photocathodes . . . . . . . . . . . . 23

3.3.1 Maximizing quantum efficiency ............ 24

3.3.2 CsI photocathode response in methane . . . . . . . . . 24

4 The XEP Time Projection Chamber and Detector Packages 26

4.1 Introduction . . . . . . . . . . . . . . 26

4.2 The drift region . . . . . . . . . . . . . . . . . . . 28

4.2 .1 Electroluminescence $(\mathrm{EL})$ region . . . . . . . . . . . . . 29

4.3 The detector packages . . . . . . . . . . . . . . . . . . 29

4.3 .1 Fused silica window . . . . . . . . . . . . 31

$4.3 .2 \mathrm{CH}_{4}$ region and $\mathrm{CsI}$ readout pads . . . . . . . . . 32

4.4 Initial detector package $\ldots \ldots \ldots . \ldots \ldots$

5 Secondary XEP Equipment $\quad 36$

5.1 Data acquisition electronics . . . . . . . . . . . . 36

5.1.1 Characterization of noise in the CAEN data acquisition system 37

5.2 Vacuum evaporation chamber . . . . . . . . . . . . . 47

5.2 .1 Uniformity of CsI evaporations f . . . . . . . . . . 48

5.3 Creating a conductive grid on fused silica using photolithography . . 53

6 Signals From the First XEP Detector Package - Results and Anal$\begin{array}{ll}\text { ysis } & 56\end{array}$

6.1 CsI response in methane . . . . . . . . . . . . . . . . . 59

6.1 .1100 Torr gain curves . . . . . . . . . . . . . 62

6.1 .220 Torr gain curves . . . . . . . . . . . . . 69 
6.2 Pad degradation . . . . . . . . . . . . . . 74

6.3 Response across a pad . . . . . . . . . . . . . . 78

6.4 Maximum gain measurement . . . . . . . . . . . . 85

$\begin{array}{llr}7 & \text { Summary and Conclusion } & \mathbf{8 9}\end{array}$

7.1 Next steps . . . . . . . . . . . . . . . . . . . . . 91

$\begin{array}{ll}\text { References } & 93\end{array}$

$\begin{array}{ll}\text { Appendix A Data Tables } & 97\end{array}$ 


\section{List of Tables}

2.1 Fundamental particles of the Standard Model and their properties . . 6

2.2 Results of several double beta decay experiments . . . . . . . . . . 14

5.1 CAEN preamplifier specifications ............... 37

5.2 CsI dispersion pattern using the 5 boat configuration $\ldots \ldots . . .50$

5.3 CsI dispersion pattern using only the central boat . . . . . . . . 51

A.1 Pulse heights for varying E/p values for pad B15 at 100 Torr . . . . 97

A.2 Pulse heights for varying E/p values for pad B22 at 100 Torr . . . . 98

A.3 Pulse heights for varying E/p values for pad B31 at 100 Torr . . . . 99

A.4 Pulse heights for varying E/p values for pad B15 at 20 Torr . . . . 100

A.5 Pulse heights for varying E/p values for pad B31 at 20 Torr . . . . 101

A.6 Pulse heights at different locations on pad C9 . . . . . . . . . . . 101

A.7 Pulse heights from the maximum gain test . . . . . . . . . . 102 


\section{List of Figures}

2.1 Normal and inverted neutrino mass hierarchies . . . . . . . . . 12

2.2 Constraints on the normal and inverted hierarchies . . . . . . . . . 12

2.3 Energy level diagrams for $(\mathrm{Z}, \mathrm{A}),(\mathrm{Z}+1, \mathrm{~A})$, and $(\mathrm{Z}+2, \mathrm{~A})$ atoms. . . 13

2.4 Generic Feynmann diagrams of $2 \nu \beta \beta$ and $0 \nu \beta \beta \ldots \ldots 15$

$2.52 \nu \beta \beta$ and $0 \nu \beta \beta$ 2-electron energy spectrum models . . . . . . . 16

2.6 Reconstructed energies for single site events from EXO-200 . . . . 18

2.7 Comparison of the $0 \nu \beta \beta$ half-life limits for xenon and germanium . . 19

3.1 Schematic of an electron avalanche . . . . . . . . . . 25

4.1 The XEP cleanroom and TPC . . . . . . . . . . . 27

4.2 A cross section of the XEP TPC . . . . . . . . . . . . 28

4.3 A section of the EL grid . . . . . . . . . . . . . . . . . 29

4.4 A cross section of the XEP detector package . . . . . . . . . . 30

4.5 Schematic of the detector package showing signal generation . . . . . 31

4.6 CsI photocathode board layout . . . . . . . . . . . . 33

4.7 Initial detector package $-\mathrm{CH}_{4}$ side Teflon spacer $\ldots \ldots . \ldots 34$

4.8 Detector package ring with window, mesh, and Teflon in place . . . 34

4.9 Photo of the actual CsI deposition for the first photocathode . . . . . 35

5.1 A sample waveform measure by the CAEN data acquisition electronics 39

5.2 A sample distribution of measured baseline means in CAEN module B0 39

5.3 A sample distribution of measured pulse heights in CAEN module B0 40 
5.4 Pulse height means and standard deviations for CAEN A2792 module

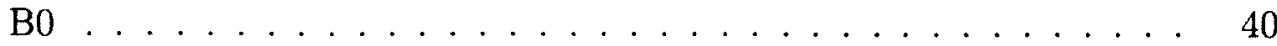

5.5 Mean and standard deviation of baseline for CAEN A2792 module B0

5.6 Pulse height means and standard deviations for CAEN A2792 module

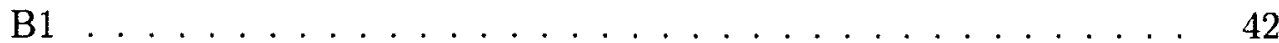

5.7 Mean and standard deviation of baseline for CAEN A2792 module B1 42

5.8 Pulse height means and standard deviations for CAEN A2792 module

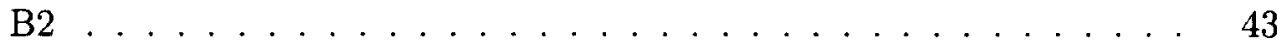

5.9 Mean and standard deviation of baseline for CAEN A2792 module B2

5.10 Pulse height means and standard deviations for CAEN A2792 module

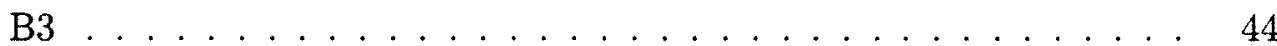

5.11 Mean and standard deviation of baseline for CAEN A2792 module B3 44

5.12 Distribution of the measured baselines for all 128 channels . . . . . . 46

5.13 Distribution of the pulse heights for all 128 channels . . . . . . . . . . 46

5.14 Cut-away view of the vacuum evaporator . . . . . . . . . . . . . . 47

5.15 Model of the 5-boat CsI dispersion pattern. . . . . . . . . . 49

5.16 Measured 5-boat CsI dispersion pattern . . . . . . . . . . . . 50

5.17 Measured 1-boat CsI dispersion pattern . . . . . . . . . . . 51

5.18 Model of the 1-boat CsI dispersion pattern . . . . . . . . . 52

5.19 Photographs of 3 successful photolithography tests . . . . . . . . 55

6.1 The set-up to hold the fibre optic cable to the detector package . . 57

6.2 The fibre optic cable placed under the Teflon openings . . . . . . 58

6.3 Schematic of the set-up used to characterize the first detector package 58

6.4 Sample pulse recorded by the Textronix oscilloscope . . . . . . . . . . 59

6.5 Successive pulse height corrections at $1111 \mathrm{~V}$ in $100 \mathrm{Torr} . . . . .661$

6.6 Pulse heights from the 100 Torr pre-run data sets . . . . . . . . . . 63

6.7 Correction factors from the 100 Torr pre-run data sets . . . . . . . 64 
6.8 Pulse heights for 3 CsI pads in 100 Torr as a function of E/p . . . . . 64

6.9 Gain curve for 3 CsI pads in 100 Torr as a function of $\mathrm{E} / \mathrm{p} \ldots$. . . . 66

6.10100 Torr gain curve focused to the no-gain region . . . . . . . . 67

6.11100 Torr gain curve with a reduced electric field gap for pad B31 . . $\quad 68$

6.12 Pad resolutions for $3 \mathrm{CsI}$ pads in 100 Torr as a function of $\mathrm{E} / \mathrm{p}$. . . . 69

6.13 Pulse heights from the 20 Torr pre-run data sets . . . . . . . . . . . 70

6.14 Corrections factors from the 20 Torr pre-run data sets . . . . . . . 71

6.15 Pulse heights for 2 CsI pads in 20 Torr as a function of $\mathrm{E} / \mathrm{p} \ldots 71$

6.16 Gain curve for 2 CsI pads in 20 Torr as a function of E/p . . . . . 72

6.1720 Torr gain curve focused to the no-gain region . . . . . . . . . 73

$6.18 \mathrm{Pad}$ resolutions for $2 \mathrm{CsI}$ pads in 20 Torr as a function of $\mathrm{E} / \mathrm{p} \ldots 74$

6.19 Drift of pulse heights over time at low light intensity . . . . . . . 75

6.20 Drift of pusle heights over time at high light intensity . . . . . . . 77

6.21 Approximate locations of measurements to examine response across a

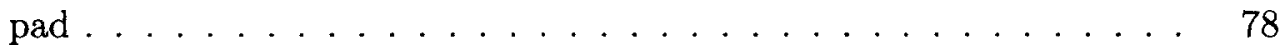

6.22 Pad-scan pulse heights at position $1 \ldots \ldots \ldots$. . . . . . . 79

6.23 Pad-scan pulse heights at position $2 \ldots \ldots \ldots$

6.24 Pad scan pulse heights at position $3 \ldots \ldots \ldots 1$

6.25 Pad-scan pulse heights at position $4 \ldots \ldots \ldots 2$

6.26 Pad-scan pulse heights at position $5 \ldots \ldots . \ldots . \ldots 8$

6.27 Pulse heights at 5 locations on a CsI pad . . . . . . . . . . 84

6.28 Pulse heights from the 20 Torr maximum gain measurement . . . . 86

6.29 Gain curve in 20 Torr up to a maximum value . . . . . . . . . . 87

$6.30 \mathrm{CsI}$ pad resolution over the course of the maximum gain test . . . . 88

6.31 CsI pad resolution focused to show the best resolution measured . . . 88 


\section{Chapter 1}

\section{Introduction}

Significant progress has been made in the past 80 years toward developing a complete understanding of neutrinos. In 1956 Clyde Cowan and Frederick Reines published their work proving the existence of the electron neutrino [1]. In 1962, with the discovery of the muon neutrino, it was shown that more than one flavour existed [2]. More recently, the phenomenon of neutrino mixing has been demonstrated $[3,4]$; this effect proves that neutrinos have non-zero mass. Despite these advancements there remain unanswered questions regarding the fundamental nature of neutrinos. For example, the neutrino oscillation experiments are able to measure differences in the squares of the masses, but provide no information about the overall mass scale. These experiments are also unable to determine which neutrino is the heaviest and which is the lightest. Another missing piece of information is whether or not neutrinos and anti-neutrinos are distinct particles or the same particle.

The Enriched Xenon Observatory, EXO for short, is an experiment endeavouring to observe the neutrinoless double beta decay of ${ }^{136} \mathrm{Xe}$. An observation of this decay will tell definitively whether or not the neutrino and anti-neutrino are distinct particles. This observation will also provide the information necessary to determine the mass scale of the neutrino. The EXO collaboration consists of more than 70 
people from 16 institutions worldwide. Effort is currently focused on the development and operation of two prototype detectors. The first, called EXO-200, is a time projection chamber which uses liquid xenon (LXe) as both its signal source and detection medium [5]. EXO-200 is currently operating at the Waste Isolation Pilot Plant (WIPP) near Carlsbad, New Mexico, and has already produced several interesting physics results. This detector is a small-scale working version of a proposed multi-tonne scale detector.

The second prototype, called the Xenon Electroluminescent Prototype (XEP), will use gaseous xenon (GXe) as its detection medium and is currently under construction at Carleton University. The purpose of this prototype is to demonstrate a potentially improved energy resolution over the LXe detector and better background event rejection through improved spatial resolution. There is also potential to extract and tag the barium daughter produced in the decay; this would provide exceptional background rejection. The development and characterization of the XEP detector will be the main focus of the work presented in this thesis.

\subsection{Outline}

In this thesis I will describe the XEP time projection chamber (TPC) and detector package as well as the physics involved in its operation. This chapter includes a description of my contributions to both EXO-200 and XEP. To understand the motivation for a project such as EXO, Chapter 2 will include an introduction to the neutrino. This involves discussions about neutrino oscillations, neutrino mass, double beta decay, neutrinoless double beta decay, and how an observation and measurement of neutrinoless double beta decay can provide information regarding the unknown neutrino properties. Chapter 3 covers the specific physics involved in the the signal generation and collection within XEP. In Chapter 4 the XEP TPC and 
its detector packages will be described in detail. Some of the XEP systems which I was particularly involved with are described in Chapter 5. Chapter 6 will describe in detail the work I have done to characterize the first XEP detector package and will include my analyses and results. All of this work will be summarized in Chapter 7 .

\subsection{Author's Contribution}

I worked to produce and characterize the first XEP detector package as the first step in the commissioning of XEP. To produce the detector package I contributed to the commissioning of a large vacuum evaporation system, developed procedures for its operation, and measured the deposition pattern produced in the chamber. In addition, during the construction and commissioning of XEP I worked to set up, characterize, and implement a subset of the data acquisition electronics to be used with the initial system configuration. I also spent time investigating the potential of using photolithography techniques to apply a series of parallel, electrically conductive aluminum lines to the fused silica window of the detector package.

In addition to my work on XEP, I have also been involved with the day-to-day operation of the EXO-200 experiment. This work has involved travelling to the WIPP site for daily maintenance and data collection shifts as well as monitoring shifts done both in Carlsbad and Ottawa. Daily shifts at WIPP involve performing calibration data collection, physics data collection, and a host of other regular preventative and emergency maintenance tasks on the detector and its systems. Monitoring shifts involve a careful watch of all of the detector systems for problems, as well as watching for any issues which may affect data quality. Originally these monitoring shifts were conducted either at the WIPP site or off-site at one of the EXO boarding houses in Carlsbad. More recently these shifts have been conducted from Carleton with a remote control centre which I set up, tested, and for which I am in charge. 


\section{Chapter 2}

\section{Theory}

\subsection{Neutrino physics}

The Standard Model (of Particle Physics) is a theory describing the electromagnetic, weak nuclear, and strong nuclear interactions of the known sub-atomic particles $[6,7]$. This theory has enjoyed many successes since its development through the 1960s and '70s, including the subsequent discovery of several previously unknown particles which it predicted to exist [8-10]. Despite these successes, neutrino mass and neutrino oscillations are something the theory did not predict. This was an interesting discovery, providing evidence that the Standard Model, the most complete, accurate, and well tested physics theory to date, is not complete.

\subsubsection{The neutrino}

The particle we now call the neutrino was first proposed in 1930 by Wolfgang Pauli. Pauli's proposal was based solely on empirical grounds as a solution to the apparent violation of conservation of energy, momentum, and angular momentum that was observed in beta decay. It had been observed that the energy spectrum of the electron emitted in beta decay was continuous. This could not be possible if the products of beta decay were just the proton and electron. So, Pauli theorized that in addition 
to the observed proton and electron there must be a third, undetected particle that carried away some energy and momentum from the decay. To remain undetected, this particle must have no electric charge. Initially, Pauli called his mysterious particle the neutron, though this name was later appropriated by Chadwick to name the neutral particle that exists in atomic nuclei. Enrico Fermi was the one to christen Pauli's particle the neutrino, a name which means 'little neutral one'. The neutrino(s) and the other known fundamental particles are listed in Table 2.1 along with some of their properties.

Neutrinos are now known to be elementary subatomic particles which belong to the lepton family and are commonly denoted by the Greek letter $\nu$. Three flavours of neutrino are known to exist. They are neutral, spin-1/2 particles and are generated in weak decays of $\mathrm{W}$ and $\mathrm{Z}$ bosons. In the case of interactions with $\mathrm{W}$ bosons, the flavour of neutrino produced corresponds to the flavour of the lepton. Their lack of charge, in addition to the fact that they only interact via the weak and gravitational forces, makes them extremely difficult to detect. For years the neutrino was assumed to be massless, but recently it has been shown that it does in fact have some small mass. This realization came from observations of oscillations from atmospheric neutrinos and in solar neutrinos travelling to Earth.

By the 1960s physicists thought they understood how stars generated energy; they fused light elements into heavier ones. An important fusion process thought to occur in the Sun was the p-p chain in which several $\nu_{e}$ are generated. Light generated at the Sun's core is subjected to absorption and re-emission many, many times during its flight to the surface. Because of this prolonged transit, the light we see can provide no information about what occurs in the core. The only way to test the fusion theory - to get a picture of the interior of the Sun - was to look for the neutrinos. It wasn't until 1968 that the Homestake experiment reported the first observation of solar neutrinos [11]. There was a problem, though, because the 
experiment measured roughly a third of the expected neutrino flux. This discrepancy was not initially taken seriously; Davis was extracting just a few atoms from his large volume of interaction medium and maybe he simply missed some. In time, however, the discrepancy gathered attention and led to what became known as the 'solar neutrino problem'.

\begin{tabular}{ccccc}
\hline Particle & Symbol & Mass $\left[\mathrm{MeV} / \mathrm{c}^{2}\right]$ & Electric Charge $[\mathrm{e}]$ & Spin \\
\hline Up & \multicolumn{4}{c}{ Quarks } \\
Down & $\mathrm{u}$ & $1.7-3.3$ & $2 / 3$ & $1 / 2$ \\
Strange & $\mathrm{d}$ & $4.1-5.8$ & $-1 / 3$ & $1 / 2$ \\
Charm & $\mathrm{s}$ & 101 & $-1 / 3$ & $1 / 2$ \\
Bottom & $\mathrm{c}$ & 1270 & $2 / 3$ & $1 / 2$ \\
Top & $\mathrm{b}$ & 4670 & $-1 / 3$ & $1 / 2$ \\
& $\mathrm{t}$ & 172000 & $2 / 3$ & $1 / 2$ \\
\hline Electron & $\mathrm{e}$ & Leptons & & \\
\hline Electron Neutrino & $\nu_{e}$ & $<2 \times 10^{-6}$ & -1 & $1 / 2$ \\
Muon & $\mu$ & 105.66 & 0 & $1 / 2$ \\
Muon Neutrino & $\nu_{\mu}$ & $<0.19$ & -1 & $1 / 2$ \\
Tau & $\tau$ & 1776.82 & 0 & $1 / 2$ \\
Tau Neutrino & $\nu_{\tau}$ & $<18.2$ & -1 & $1 / 2$ \\
& & Force Carriers & 0 & $1 / 2$ \\
\hline & & (Bosons) & & \\
\hline Z Boson (Weak Force) & $\mathrm{Z}$ & 91187.6 & 0 & 1 \\
\hline Photon (EM Force) & $\gamma$ & 0 & 0 & 1 \\
G Buon (Strong Force) & $\mathrm{g}$ & 0 & 0 & 1 \\
\hline
\end{tabular}

Table 2.1: The fundamental particles of the Standard Model along with some of their properties [12]. 


\subsubsection{Neutrino oscillations}

At the turn of the century the Super-Kamiokande project proved that neutrinos have mass by measuring neutrino oscillations with atmospheric neutrinos [3]. This phenomenon was confirmed by the SNO experiment with solar neutrinos [4]. The SNO experiment differed from Homestake in that SNO was sensitive to all 3 neutrino flavours, where Homestake was sensitive to just $\nu_{e}$. Since SNO were able to observe the three flavours, they were able to account for the deficit measured by Davis at Homestake. The total measured solar neutrino flux matched predictions. It was then clear that neutrinos can change flavour as they propagate and the mechanism for this change was named neutrino oscillation.

But how are these flavour changes possible, why are they called 'oscillations', and how might we visualize the process? Adopting an approach such as the one used by Griffiths [13], for example, we imagine a simplified case where there are only 2 neutrino flavours. Since we know that the transition $\nu_{e} \leftrightarrow \nu_{\mu}$ is possible, neither flavour state is a state of definite mass; one would say that these weak flavour eigenstates are different than the mass eigenstates. We can therefore define linear orthogonal combinations of the mass eigenstates to describe the flavour eigenstates. We can write these states as follows:

$$
\nu_{e}=\cos (\theta) \nu_{1}+\sin (\theta) \nu_{2}: \nu_{\mu}=-\sin (\theta) \nu_{1}+\cos (\theta) \nu_{2}
$$

Now if we take a particle that starts out as a $\nu_{e}$, it can be shown [14] that the probability that a $\nu_{e}$ may be measured to be a $\nu_{\mu}$ is:

$$
P_{\nu_{e} \rightarrow \nu_{\mu}}=\sin ^{2}(2 \theta) \sin ^{2}\left(1.27 \Delta m_{21}^{2} \frac{L}{E}\right)
$$

where $\theta$ is the mixing angle, $\Delta \mathrm{m}_{21}^{2}=\mathrm{m}_{2}^{2}-\mathrm{m}_{1}^{2}, \mathrm{E}[\mathrm{MeV}]$ is the energy of the neutrino, and $\mathrm{L}[\mathrm{m}]$ is the distance travelled by the neutrino. Because this function can be 
nonzero if the angle $\theta$ is larger than zero, one can see that the $\nu_{e}$ has some probability of being measured as a $\nu_{\mu}$, and that this probability varies sinusoidally. This is the origin of the term 'neutrino oscillation'. One can also see that the probability is proportional to the difference in the squares of the masses of the mass eigenstates. Alternatively, the mass eigenstates can be defined as linear orthogonal combinations of the weak flavour eigenstates.

In the three neutrino picture, the weak flavour states are connected to the mass eigenstates by the Pontecorvo-Maki-Nakagawa-Sakata (PMNS) matrix, often also known as the neutrino mixing matrix or the MNS matrix. The PMNS matrix is denoted by $\mathrm{U}$ and is given as follows [12]:

$$
U=\left[\begin{array}{ccc}
U_{e 1} & U_{e 2} & U_{e 3} \\
U_{\mu 1} & U_{\mu 2} & U_{\mu 3} \\
U_{\tau 1} & U_{\tau 2} & U_{\tau 3}
\end{array}\right]
$$

with a row for each flavour of lepton. The flavour eigenstates can be written in terms of the mass eigenstates as follows:

$$
\left[\begin{array}{c}
\nu_{e} \\
\nu_{\mu} \\
\nu_{\tau}
\end{array}\right]=\left[\begin{array}{ccc}
U_{e 1} & U_{e 2} & U_{e 3} \\
U_{\mu 1} & U_{\mu 2} & U_{\mu 3} \\
U_{\tau 1} & U_{\tau 2} & U_{\tau 3}
\end{array}\right]\left[\begin{array}{c}
\nu_{1} \\
\nu_{2} \\
\nu_{3}
\end{array}\right]
$$

where $\nu_{n}(\mathrm{n}=1,2$, or 3$)$ are the mass eigenstates. The flavour eigenstates are then given as linear combinations of the mass eigenstates as follows:

$$
\nu_{e}=U_{e 1} \nu_{1}+U_{e 2} \nu_{2}+U_{e 3} \nu_{3}
$$




$$
\begin{aligned}
& \nu_{\mu}=U_{\mu 1} \nu_{1}+U_{\mu 2} \nu_{2}+U_{\mu 3} \nu_{3} \\
& \nu_{\tau}=U_{\tau 1} \nu_{1}+U_{\tau 2} \nu_{2}+U_{\tau 3} \nu_{3} .
\end{aligned}
$$

The common parametrization of this unitary matrix is the following:

$$
U=\left[\begin{array}{ccc}
c_{12} c_{13} & s_{12} c_{13} & s_{13} e^{-\imath \delta} \\
-s_{12} c_{23}-c_{12} s_{23} s_{13} e^{\imath \delta} & c_{12} c_{23}-s_{12} s_{23} s_{13} e^{\imath \delta} & s_{23} c_{13} \\
s_{12} s_{23}-c_{12} c_{23} s_{13} e^{\imath \delta} & -c_{12} s_{23}-s_{12} c_{23} s_{13} e^{\imath \delta} & c_{23} c_{13}
\end{array}\right] \times \operatorname{diag}\left(1, e^{2 \frac{\alpha_{21}}{2}}, e^{\imath \frac{\alpha_{31}}{2}}\right)
$$

The $s_{i j}\left(c_{i j}\right)$ here are used to represent the sine (cosine) of angle $\theta_{i j}$, which is bound between 0 and $\frac{\pi}{2}$. In addition, $\delta$ is the Dirac charge-parity (CP) violation phase, while $\alpha_{21}$ and $\alpha_{31}$ are Majorana CP violation phases. Current data constrains $\mathrm{U}$ fairly well, suggesting the values $\theta_{12} \cong \frac{\pi}{5.4}, \theta_{13}<\frac{\pi}{13}$, and $\theta_{23} \cong \frac{\pi}{4}$ [12]. A recent result from the Daya Bay experiment gives an improved measurement of $\theta_{13}$, with the value $\theta_{13} \cong \frac{\pi}{40.8}[15]$. The angles $\theta_{12}$ and $\theta_{23}$ are often also known as $\theta_{s o l}$ and $\theta_{a t m}$ respectively, where 'sol' means solar and 'atm' means atmospheric.

As stated above, the oscillation probability is related to the difference of the squares of the masses, and neutrino oscillation experiments have provided measurements of these values for the three mass eigenstates. These differences are $\Delta m_{21}^{2} \equiv m_{2}^{2}-m_{1}^{2}=\Delta m_{\text {sol }}^{2}$ and $\Delta m_{32}^{2} \equiv m_{3}^{2}-m_{2}^{2}=\Delta m_{\text {atm }}^{2} . \quad \Delta m_{21}^{2}$ has been measured by the SNO experiment, for example, with the result $\Delta m_{21}^{2}=7.41_{-0.21}^{+0.19}$ $\mathrm{x} 10^{-5} \mathrm{eV}^{2}$ [16]. $\Delta m_{32}^{2}$ has been measured by the MINOS experiment, for example, with the result $\Delta m_{32}^{2}=2.62_{-0.28}^{+0.31}$ (stat.) \pm 0.09 (sys.) $\times 10^{-3} \mathrm{eV}^{2}$ [17]. The mass squared differences, however, can provide no information about the absolute mass scale. 


\subsubsection{Neutrino mass}

Currently, as seen in Table 2.1, there are only upper limits on the neutrino masses. Each of these limits is set a different way. The tau neutrino mass limit is set by measuring the shape of the energy spectrum of decay products of tau leptons and limiting the phase space for the neutrino mass, as described in [18]. There are two decay channels which are measured:

$$
\tau^{-} \rightarrow 3 \pi^{-}+2 \pi^{+}+\nu_{\tau}
$$

and

$$
\tau^{-} \rightarrow 2 \pi^{-}+\pi^{+}+\nu_{\tau}
$$

The muon neutrino mass limit (Table 2.1) is set by measuring the momentum of muons produced in pion decays, such as by [19]. This decay proceeds as follows:

$$
\pi^{+} \rightarrow \mu^{+}+\nu_{\mu}
$$

The electron neutrino mass limit (Table 2.1) is set by measuring the electron energy spectrum in the beta decay of tritium. Tritium is a radioactive isotope of hydrogen, denoted ${ }^{3} \mathrm{H}$, whose nucleus contains one proton and two neutrons. The decay proceeds as follows:

$$
{ }_{1}^{3} H_{2} \rightarrow{ }_{2}^{3} H e_{1}^{+}+e+\bar{\nu}_{e}
$$

Tritium decay provides one of the most sensitive probes of the neutrino mass. A new experiment called KATRIN [20] is currently under construction and will attempt to improve the electron neutrino mass limit set by experiments such as Mainz [21]. The goal of KATRIN is an improvement of 1 order of magnitude over the current 
limit, down to $0.2 \mathrm{eV}$.

Another way to measure the neutrino mass scale is through the observation of a theoretical process called neutrinoless double beta decay. A measurement of the half-life of neutrinoless double beta decay would provide the final piece of information needed to calculate the effective electron neutrino mass. The rate of $0 \nu \beta \beta$ is given by $[22]$ :

$$
\left(T_{1 / 2}^{0 \nu}\right)^{-1}=G_{0 \nu}\left(Q_{\beta \beta}, Z\right)\left|M_{0 \nu}\right|^{2}\left\langle m_{\beta \beta}\right\rangle^{2}
$$

Here, $G_{0 \nu}\left(Q_{\beta \beta}, Z\right)$ is a phase space factor which is exactly calculable, $\left|M_{0 \nu}\right|^{2}$ is a matrix element which is model dependent, and $\left\langle m_{\beta \beta}\right\rangle$ is the effective Majorana mass of $\nu_{e}$. The quantity $\left\langle m_{\beta \beta}\right\rangle$ is defined as follows [22]:

$$
\left\langle m_{\beta \beta}\right\rangle \equiv\left|\sum_{k} m_{k} U_{e k}^{2}\right|
$$

Hence the effective electron neutrino mass is a linear combination of the mass eigenstates $\mathrm{m}_{k}$ with the $\mathrm{U}_{e k}^{2}$ coefficients defined by the PMNS matrix. A lower limit for $\mathrm{T}_{1 / 2}^{0 \nu}$ in ${ }^{136} \mathrm{Xe}$ recently set by EXO-200 gives an upper limit on the effective $\nu_{e}$ mass of $140-380 \mathrm{meV}$ [23] depending on the matrix element used (see Figure 2.7).

The neutrino mass hierarchy, shown schematically in Figure 2.1, describes the mass spectrum of $m_{1}, m_{2}$, and $m_{3}$. The actual ordering of the masses is unknown and there are two schemes describing it. The first, normal hierarchy, has $m_{1}<m_{2}<m_{3}$. The second, inverted hierarchy, has $m_{3}<m_{1}<m_{2}$. For an effective electron neutrino mass above about $100 \mathrm{meV}$ the two hierarchies are indistinguishable and the mass spectrum is said to be degenerate. Both hierarchies are constrained, as shown in Figure 2.2, by measurements of neutrino oscillations. 


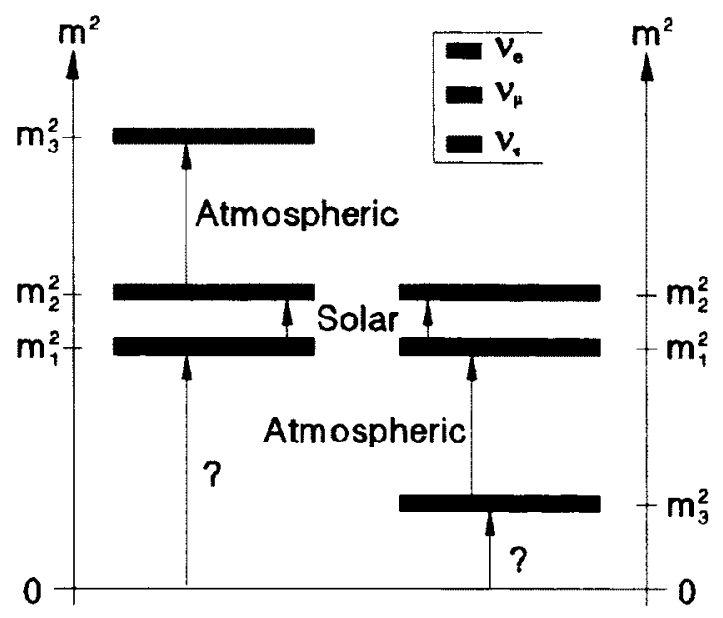

Figure 2.1: The two mass hierarchies are shown, with the normal hierarchy on the left and the inverted hierarchy on the right. The coloured regions indicate the flavour content of each of the mass states.

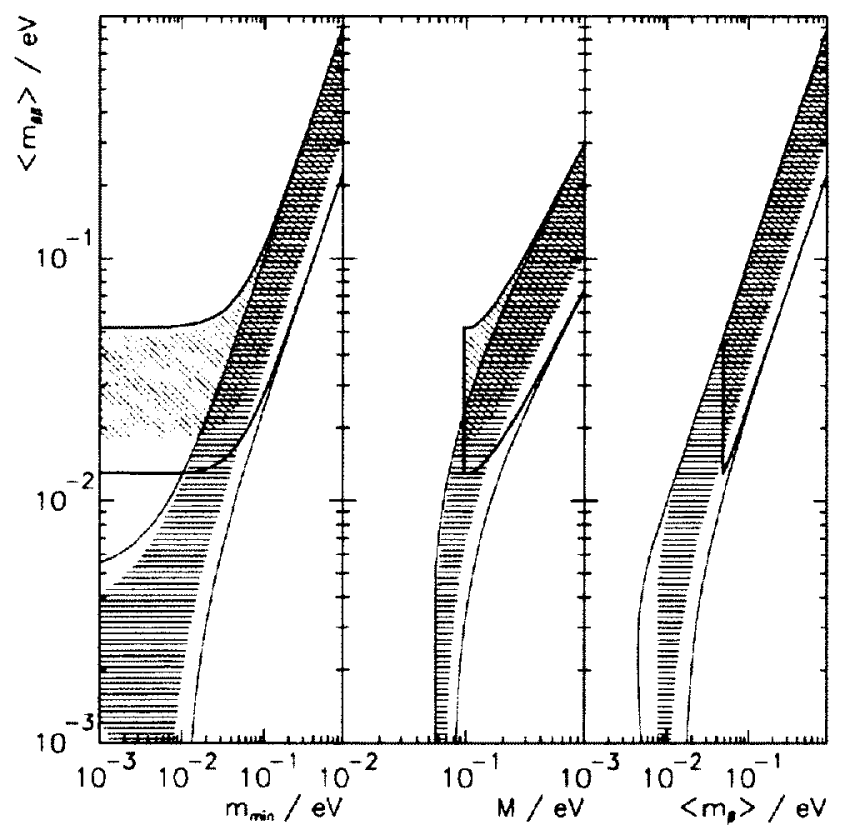

Figure 2.2: The solid lines show the ranges of the two hierarchies, which merge for $\left\langle m_{\beta \beta}\right\rangle>100 \mathrm{meV}$ where they are indistinguishable. Left panel: the relation between $\left\langle m_{\beta \beta}\right\rangle$ and the mass of the lightest neutrino. Middle panel: $\left\langle m_{\beta \beta}\right\rangle$ as a function of the sum of neutrino masses M. Right panel: $\left\langle m_{\beta \beta}\right\rangle$ as a function of the average neutrino mass, $\left\langle m_{\beta}\right\rangle$, where $\left\langle m_{\beta}\right\rangle=\left[\Sigma\left|U_{e k}\right|^{2} m_{k}^{2}\right]^{1 / 2}[24]$. 


\subsection{Double beta decay}

Double beta decay $(2 \nu \beta \beta)$ is a process whereby two neutrons are simultaneously converted to two protons, two electrons, and two electron anti-neutrinos. Generally, this process looks like:

$$
{ }_{Z}^{A} X_{N} \rightarrow{ }_{Z+2}^{A} Y_{N-2}+2 e+2 \bar{\nu}_{e}
$$

This process can only occur in nuclei with an even number of protons $(Z)$ and neutrons $(N)$ which are either energetically forbidden from undergoing (single) beta decay, or where beta decay is highly suppressed. From an energy level perspective, the $(\mathrm{Z}, \mathrm{A})$ level is lower than the $(\mathrm{Z}+1, \mathrm{~A})$ atom's, but higher than the $(\mathrm{Z}+2, \mathrm{~A})$ atom's. This is shown schematically in Figure 2.3.

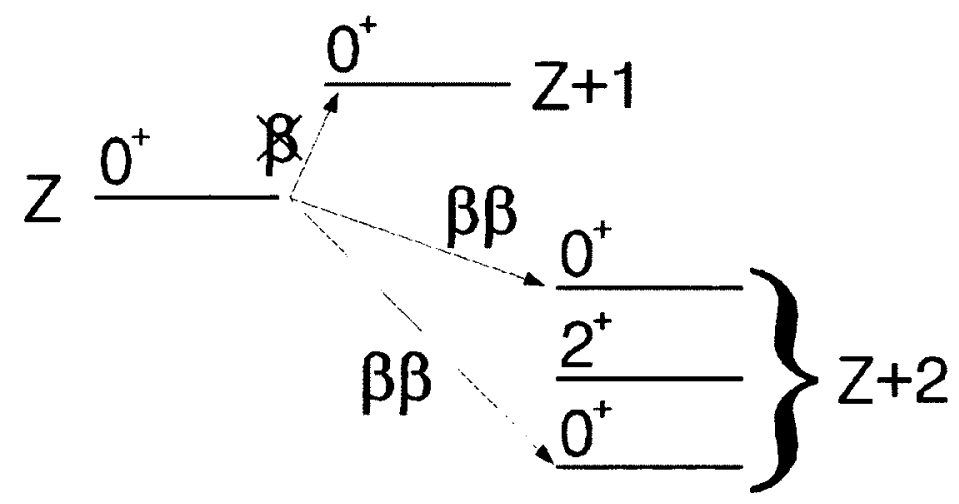

Figure 2.3: Energy level diagrams for $(Z, A),(Z+1, A)$, and $(Z+2, A)$ atoms. The beta decay transition from $(\mathrm{Z}, \mathrm{A})$ to $(\mathrm{Z}+1, \mathrm{~A})$ is forbidden as the $\mathrm{Z}+1$ nucleus is less tightly bound. The same is not true for the $Z+2$ nucleus and double beta decay may occur. The normal transition is from the $0+$ nuclear angular momentum state of the parent to the $0+$ ground state (lowest energy) of the daughter. The transition to an excited $0+$ state of the daughter is possible, but is highly suppressed.

Only a small number of isotopes have the potential to undergo this decay. An even smaller number of those isotopes have had their $2 \nu \beta \beta$ half-lives measured. These 
measurements are summarized in Table 2.2.

\begin{tabular}{cc}
\hline Isotope & $T_{1 / 2}^{2 \nu}[$ years $]$ \\
\hline${ }^{48} \mathrm{Ca}$ & $\left(4.2_{-1.0}^{+2.1}\right) \times 10^{19}$ \\
${ }^{76} \mathrm{Ge}$ & $(1.5 \pm 0.1) \times 10^{21}$ \\
${ }^{82} \mathrm{Se}$ & $(0.92 \pm 0.07) \times 10^{20}$ \\
${ }^{96} \mathrm{Zr}$ & $(2.0 \pm 0.3) \times 10^{19}$ \\
${ }^{100} \mathrm{Mo}$ & $(7.1 \pm 0.4) \times 10^{18}$ \\
${ }^{116} \mathrm{Cd}$ & $(3.0 \pm 0.2) \times 10^{19}$ \\
${ }^{128} \mathrm{Te}$ & $(2.5 \pm 0.3) \times 10^{24}$ \\
${ }^{130} \mathrm{Ba}$ & $(2.2 \pm 0.5) \times 10^{21}$ \\
${ }^{130} \mathrm{Te}$ & $(0.9 \pm 0.1) \times 10^{21}$ \\
${ }^{150} \mathrm{Nd}$ & $(7.8 \pm 0.7) \times 10^{18}$ \\
${ }^{238} \mathrm{U}$ & $(2.0 \pm 0.6) \times 10^{21}$ \\
\hline${ }^{136} \mathrm{Xe}(\mathrm{EXO}, 2011)$ & $2.11 \pm 0.04($ stat $) \pm 0.21($ sys. $) \times 10^{21}$ \\
${ }^{136} \mathrm{Xe}(\mathrm{KamLAND}-\mathrm{Zen}, 2011)$ & $2.38 \pm 0.02($ stat $) \pm 0.14($ syst $) \times 10^{21}$ \\
${ }^{136} \mathrm{Xe}(\mathrm{EXO}, 2012)$ & $2.23 \pm 0.017$ (stat) $\pm 0.22($ sys. $) \times 10^{21}$ \\
\hline
\end{tabular}

Table 2.2: Measured $2 \nu \beta \beta$ half-lives for several isotopes. All values, apart from ${ }^{136} \mathrm{Xe}$, are averages calculated by Barabash [25]. The ${ }^{136} \mathrm{Xe}$ half-life was measured by EXO (2011) [26], EXO (2012) [23], and KamLAND-Zen [27]. All three measurements are consistent.

In contrast, as its name states, neutrinoless double beta decay $(0 \nu \beta \beta)$ does not involve the emission of two neutrinos. In $0 \nu \beta \beta$ the neutrinos are purely virtual particles which annihilate each other during the process. Generic Feynmann diagrams for both processes are shown in Figure 2.4. The $0 \nu \beta \beta$ process is an exotic form of double beta decay, and to date its existence has not been independently confirmed. A sub-group of the Heidelberg-Moscow experiment, led by H.V. Klapdor-Kleingrothaus, claims an observation of this process in ${ }^{76} \mathrm{Ge}$ with $\mathrm{T}_{1 / 2}^{0 \nu}=2.23_{-0.31}^{+0.44} \times 10^{25}$ years to a significance of $6.4 \sigma[28]$. The Klapdor measurement has never been reproduced.

$0 \nu \beta \beta$ can only occur if two conditions are met. The first condition is that the 
neutrino must have mass; we know this to be true. The second condition is that the neutrino must be its own anti-particle. Most particles have a distinct corresponding anti-particle; these types of particles are known as Dirac particles. Some particles, however, do not have a distinct anti-particle and are considered to be their own antiparticle; these types of particles are known as Majorana particles. Since the electric charge of an anti-particle is opposite its corresponding particle, all Majorana particles are neutral. There are currently no known Majorana fermions. The nature (Majorana or Dirac) of neutrinos is unknown, though an observation of $0 \nu \beta \beta$ would establish a Majorana component to the neutrino.

All leptons are assigned a quantity known as 'lepton number'. Leptons are assigned a value of +1 , and anti-leptons are assigned a value of -1 . Specifically, these numbers are restricted to the generation of lepton, meaning the +1 of an electron is not the same as $a+1$ of a muon, for example. Since the anti-neutrinos are not present to balance the electrons in $0 \nu \beta \beta$, lepton number conservation is violated by 2 units. This violation is not allowed in the Standard Model, and an observation of this process would be an indication that the model requires some modifications.
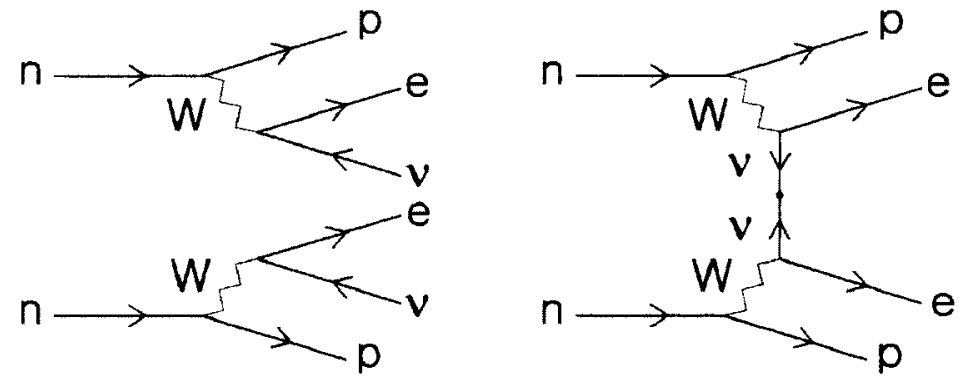

Figure 2.4: Generic Feynmann diagrams showing double beta decay (left) and neutrinoless double beta decay (right). 


\subsubsection{Electron energy spectrum}

The energy released in a nuclear decay, known as the $Q$ value, is the difference in binding energies between the parent and daughter nuclei. In the case of $2 \nu \beta \beta$ this energy is shared between the 5 products. For xenon, the recoil energy of the barium daughter is negligible because it is roughly 125,000 times heavier than the electron and neutrino pairs. The decay energy is therefore shared between the electrons and the neutrinos. Since the neutrinos can carry off some energy, the combined kinetic energy of the 2 electrons - something which can be easily measured - has a continuous spectrum of values beginning at zero and terminating at the $\mathrm{Q}$ value. In the $0 \nu \beta \beta$ case, the electrons take all of the decay energy, producing a spike at the $Q$ value. Models of these two spectra are shown together in Figure 2.5.

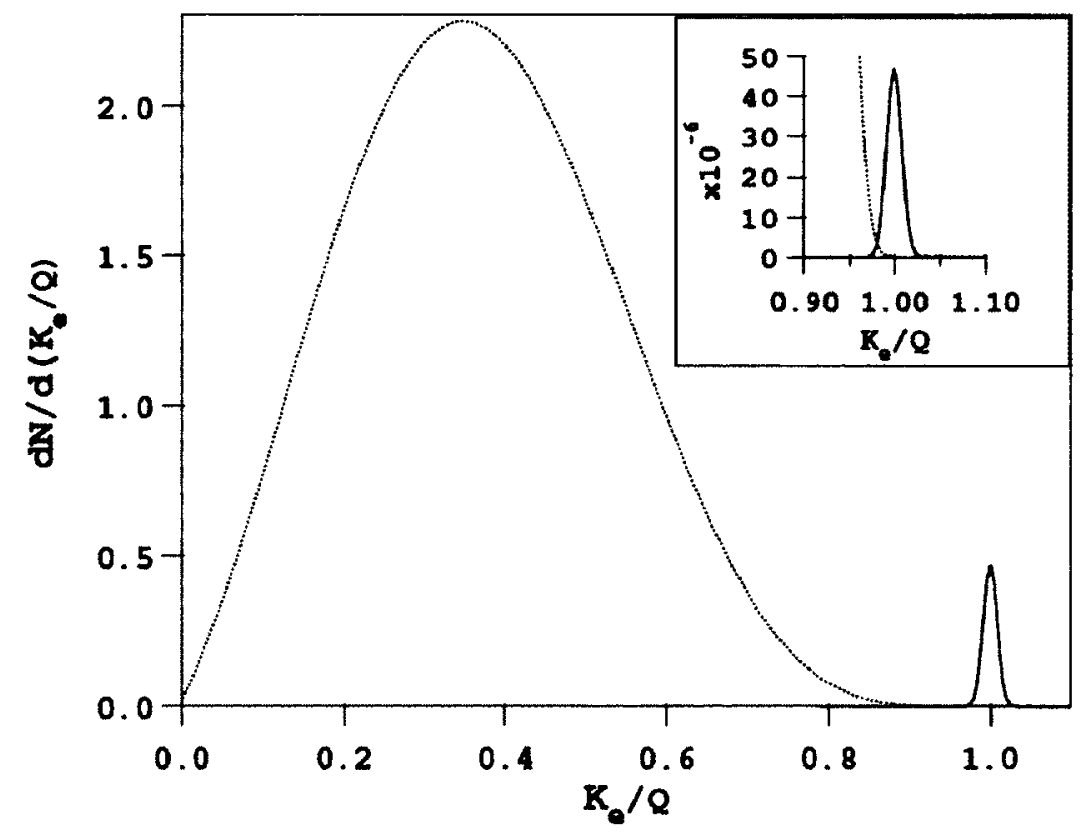

Figure 2.5: A model of a normalized 2-electron kinetic energy $\left(\mathrm{K}_{e}\right)$ spectrum for $2 \nu \beta \beta$ and $0 \nu \beta \beta$ drawn with a $2 \%$ energy resolution. From [29] "The assumed $2 \nu / 0 \nu$ rate ratio is $10^{2}$, and $10^{6}$ in the insert". 


\subsubsection{Double beta decay of ${ }^{136} \mathrm{Xe}$}

While there are a handful of known double beta decay emitters, there are a number of reasons which make ${ }^{136} \mathrm{Xe}$ an attractive candidate to search for $0 \nu \beta \beta$. Firstly, the double beta decay $Q$ value is quite high at $2457.8 \mathrm{keV}$. This energy region is relatively free from naturally occurring $\gamma$ radiation. In addition, ${ }^{136} \mathrm{Xe}$ has a natural abundance of about $8.9 \%$ and it is relatively easy to isotropically enrich in a centrifuge. It can also be purified as needed during an experiment by flowing it out of the detector, through a purifier, and back into the detector. This stands in contrast with other isotopes such as ${ }^{76} \mathrm{Ge}$ or ${ }^{128} \mathrm{Te}$ which must be grown in crystals and so cannot undergo further purification.

Furthermore, a detector running with GXe is simpler in concept than one running with LXe, and there are a number of reasons for this. To begin with, the GXe system does not require the cryogenic systems that the LXe system does. This includes fridges to maintain liquid temperature, vacuum plumbing, and monitoring systems. A gas system may also add xenon over time as its relative rarity in the atmosphere makes collection of large volumes a lengthy process. A gradual addition of xenon to a liquid system would be much more difficult to achieve. In principle, a GXe can achieve better energy resolution than a LXe detector, and would also have little or no anti-correlation between ionization and scintillation signals. Furthermore, an electron with an energy of, say, $1 \mathrm{MeV}$ travels much further through gas than through liquid. This would lead to better track reconstruction, and hence better background rejection through improved position resolution.

The $2 \nu \beta \beta$ process in ${ }^{136} \mathrm{Xe}$ proceeds as follows:

$$
{ }_{54}^{136} X e_{82} \rightarrow{ }_{56}^{136} B a_{80}^{++}+2 e+2 \bar{\nu}_{e}
$$

This decay was first observed using the EXO-200 detector in the fall of 2011. The 
measured half-life, $\mathrm{T}_{1 / 2}^{2 \nu}=2.11 \times 10^{21}$ years (Table 2.2 ), was lower than the previously published lower limit of $1.0 \times 10^{22}$ years [30]. While this was somewhat troubling, the EXO measurement was soon confirmed by the KamLAND-Zen group. More recently, a refined measurement of the $2 \nu \beta \beta$ half-life was performed by the EXO group with a resulting $\mathrm{T}_{1 / 2}^{2 \nu}$ of $2.23 \times 10^{21}$ years, in agreement with the other two measurements. The reconstructed energies of single-site events are shown in Figure 2.6. The $Q$ value for this decay has been measured to be $2457.8 \mathrm{keV}$ [31].

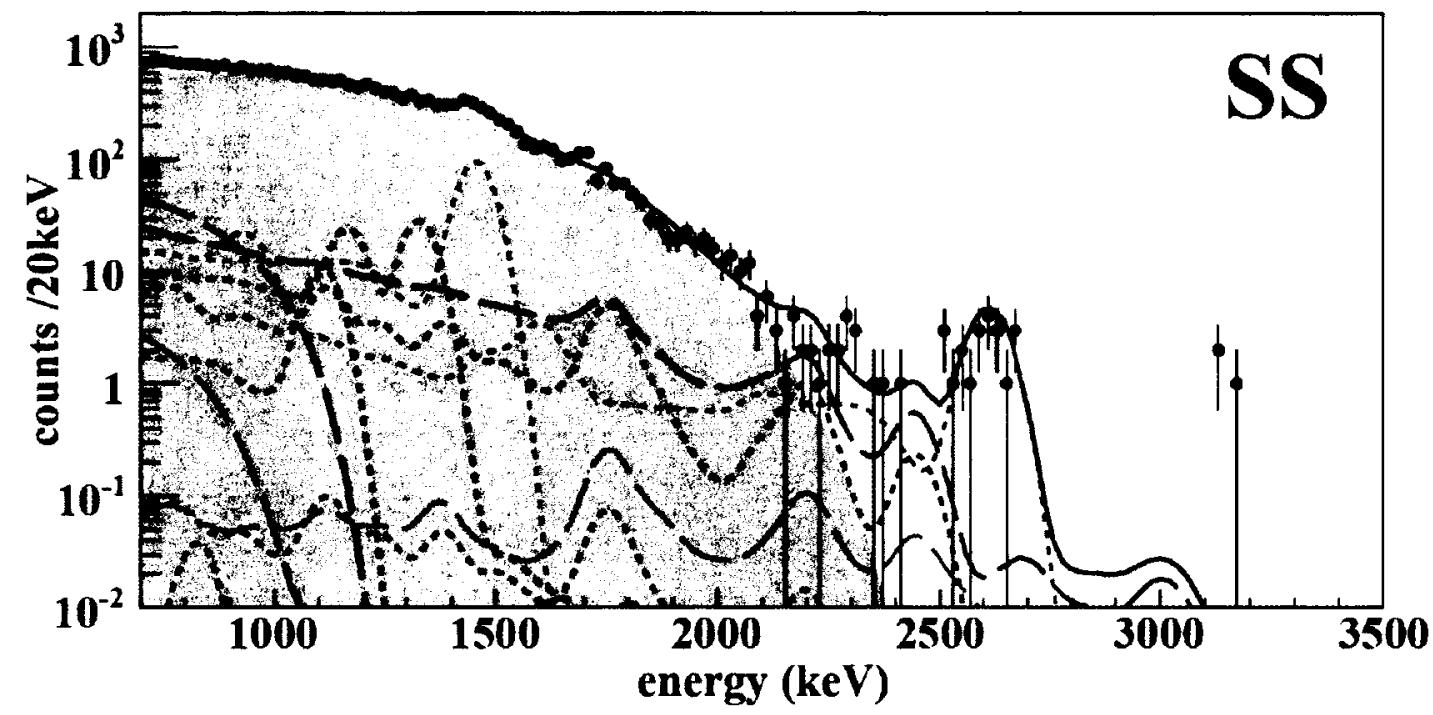

Figure 2.6: Reconstructed energies from events determined to be from a single site at EXO-200. The solid blue line is the best fit to the data, and the shaded grey area is the $2 \nu \beta \beta$ signal. The backgrounds are as follows: ${ }^{40} \mathrm{~K}$ (dotted orange), ${ }^{60} \mathrm{Co}$ (dotted dark blue), ${ }^{222} \mathrm{Rn}$ in air between the lead wall and the cryostat (long-dashed green), ${ }^{222} \mathrm{Rn}$ in the active xenon (long-dashed brown), ${ }^{222} \mathrm{Rn}$ outside of the field cage (dotted dark cyan), ${ }^{238} \mathrm{U}$ (dotted black), ${ }^{232} \mathrm{Th}$ (dotted magenta), ${ }^{214} \mathrm{Bi}$ (long-dashed cyan), ${ }^{135} \mathrm{Xe}$ (long-dashed blue), and ${ }^{54} \mathrm{Mn}$ (dotted brown) [23].

In addition to measurements of the $2 \nu \beta \beta$ half-life, both the KamLAND-Zen and EXO groups have been able to set lower limits on the $0 \nu \beta \beta$ half-life. The first limit was set by KamLAND-Zen, with $\mathrm{T}_{1 / 2}^{0 \nu}>5.7 \times 10^{24}$ years at $90 \% \mathrm{CL}$ [27]. The next limit was set by EXO-200 in conjunction with the recent $2 \nu \beta \beta$ half-life measurement. 
EXO-200 found $\mathrm{T}_{1 / 2}^{0 \nu}>1.6 \times 10^{25}$ years at $90 \% \mathrm{CL}$ [23], a factor of $\sim 3$ improvement over the KamLAND-Zen measurement. These two results are shown together with the Klapdor claim for ${ }^{76} \mathrm{Ge}$ in Figure 2.7, along with possible $\nu_{e}$ masses for different matrix elements.

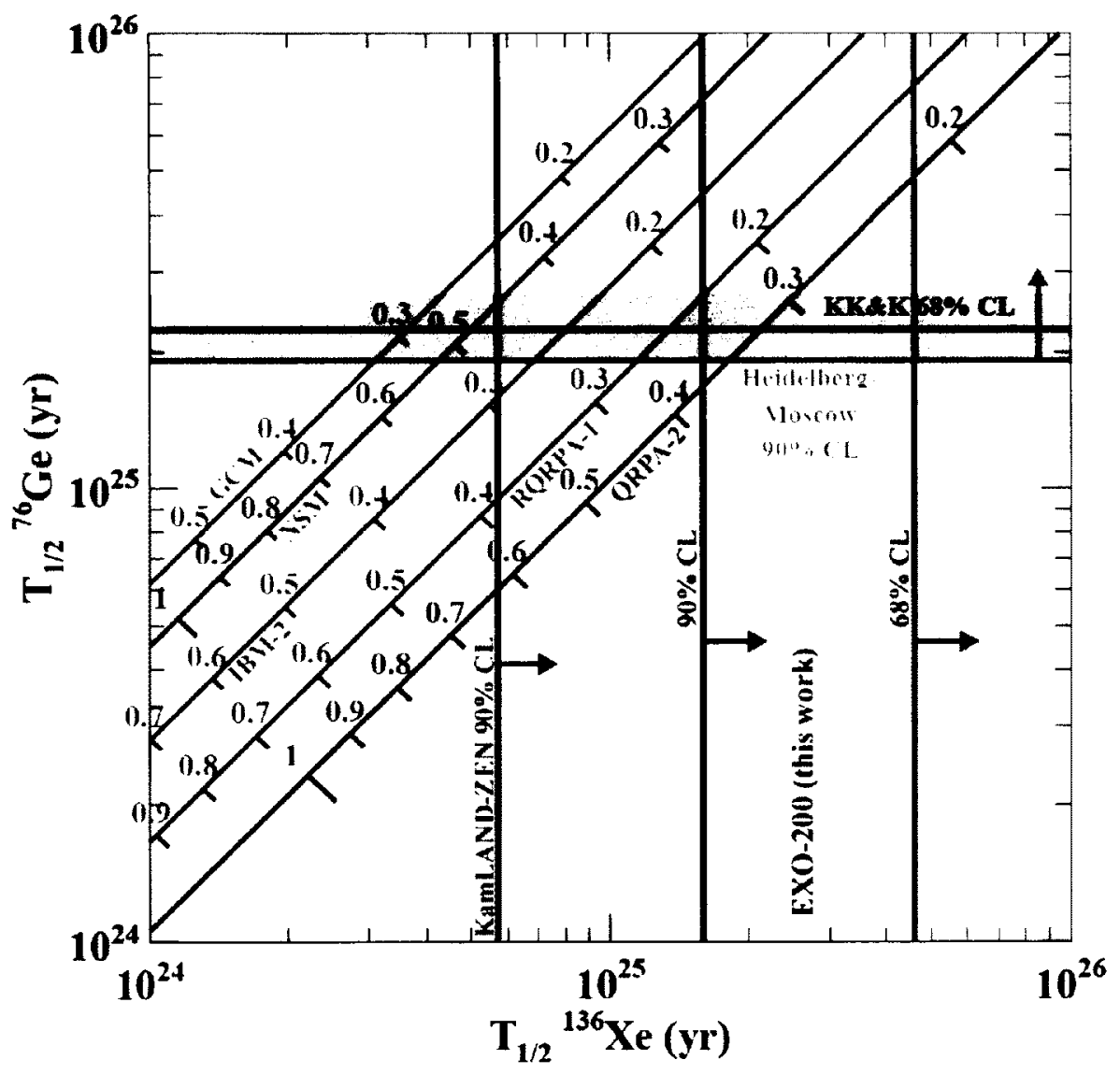

Figure 2.7: The limits set by KamLAND-Zen and EXO-200 in xenon (vertical) are shown together with the Heidelberg-Moscow limit and the Klapdor claim in germanium (horizontal). The diagonal lines show various matrix element calculations (GCM [32], NSM [33], IBM-2 [34], RQRPA-1 [35], and QRPA-2 [36]) with the ticks and numbers showing effective $\nu_{e}$ masses (in $\mathrm{eV}$ ) corresponding to the half-lives [23]. 


\section{Chapter 3}

\section{Signal Generation and Detection in XEP}

\subsection{Overview}

This chapter will provide an overview of how signals are generated and measured in XEP. A double beta decay of ${ }^{136} \mathrm{Xe}$ will produce 2 free electrons, the primary generators of the signal we will measure. Once free, these primary electrons will travel through an electric field region where they will lose energy by ionizing the gas and stimulating the production of scintillation photons. Collection of these scintillation photons will provide the initial time of the event. The ionization electrons will drift to a high electric field region where they will gain enough energy to generate secondary scintillation photons through a process called electroluminescence. The time of the collection of these electroluminescence photons compared to the arrival time of the first scintillation photons will provide the drift time of the electrons. If the drift velocity of the electrons is known, the drift time can be used to calculate the length of the drift path of the electrons through the TPC. We can label the drift direction of the electrons as the Z-axis.

When light strikes the photon detector, electrons will be liberated from the surface through the photoelectric effect; these electrons are known as photoelectrons. The photoelectrons will drift in an electric field (separate from the field drifting the 
ionization electrons) and will induce a positive signal on the photon detector. This signal will be what is measured, and will be proportional to the energy of primary electrons. In this way the energy of the primary electrons produced in a double beta decay will be measured.

The drift length, along with the $\mathrm{X}-\mathrm{Y}$ position of the electrons, will allow for the determination of the spatial location of the decay within the detector. The X$\mathrm{Y}$ position can be determined if the photon detector used to collect primary and secondary scintillation light is divided into a grid in $\mathrm{X}$ and $\mathrm{Y}$. It will be very important in XEP that the xenon is of high purity. Gas purity is crucial to XEP's energy resolution as impurities can reduce the scintillation and ionization yields.

\subsection{Ionization and scintillation}

In xenon, ionizing radiation must deposit about $22 \mathrm{eV}$ of energy to remove an electron from an atom. An electron with $1 \mathrm{MeV}$ of kinetic energy, for example, will produce $1 \times 10^{6} \mathrm{eV} / 22 \mathrm{eV} \approx 45,000$ ionization electrons.

Another way that an electron can deposit energy in a gas is through the process of scintillation. To begin, a free electron collides with and excites an atom (Eq. 3.1). Alternatively, an electron can recombine with an ion to form an excited atom (Eq. 3.2). This excited atom can then triple collide with two other atoms to form an excited dimer (Eq. 3.3), also known as an excimer. An excimer is a weakly bound state of two atoms with a decay time on the order of nanoseconds (in Xe, for example) to microseconds (in $\mathrm{Kr}$ and $\mathrm{Ar}$, for example). Finally, the excimer de-excites though the release of a characteristic photon (Eq. 3.4)

$$
\begin{gathered}
e+A \rightarrow e+A^{*} \\
e+A^{+} \rightarrow A^{*}
\end{gathered}
$$




$$
\begin{gathered}
A^{*}+2 A \rightarrow A_{2}^{*}+A \\
A_{2}^{*} \rightarrow 2 A+\gamma_{S C} .
\end{gathered}
$$

This process only occurs for gas densities in excess of $10^{10}$ atoms $\cdot \mathrm{cm}^{-3}$ [37], and photons are generated isotropically. A similar process called electroluminescence (EL), also known as secondary scintillation, is one of proportional scintillation and also produces photons with an isotropic distribution. The mechanism for the production of EL photons is the same as scintillation. For electrons drifting through a gas in an electric field the EL process is proportional to both the drift field strength and the pressure of the gas. The electroluminescence yield, $Y$, in photons per $1 \mathrm{~cm}$ of drift has been found to be [38]:

$$
Y=(140 E / p-116) p
$$

where $\mathrm{E}$ is the field strength in $\mathrm{kV} \cdot \mathrm{cm}^{-1}$ and $\mathrm{p}$ is the pressure in bar. More precisely, the EL process is proportional to the reduced electric field, E/p $\left[\mathrm{kV} \cdot \mathrm{cm}^{-1} \cdot \mathrm{bar}^{-1}\right]$. The process of electroluminescence begins then at a threshold of roughly $0.83 \mathrm{kV} \cdot \mathrm{cm}^{-1} \cdot$ bar $^{-1}$, thus a minimum field of $8.3 \mathrm{kV} \cdot \mathrm{cm}^{-1}$ is required at 10 bar. Below this threshold, electrons cannot gain enough energy to excite the atoms.

The scintillation process in xenon generates VUV photons at wavelengths centered around $147 \mathrm{~nm}$ [38] and $173 \mathrm{~nm}$ [39], known as the first and second continua, respectively. The first continuum is generated by the radiative decay of vibrationally excited excimers, and the second by vibrationally relaxed excimers. The first continuum is highly suppressed, however, if the pressure is greater than 400 mbar; above this pressure the second continuum dominates, producing a peak centered around 173 $\mathrm{nm}$ and with a full width at half maximum of about $14 \mathrm{~nm}$ [39]. 
A medium is essentially transparent to any EL photons it produces, so these photons can be detected some distance away from the site of their generation. This process contributes negligible statistical fluctuations in the photon yield when compared to fluctuations in the number of initial electrons [40]. Because of this fact, it is possible to build an EL detector to measure electron energies which has very good intrinsic energy resolution.

The Fano factor, F, describes statistical variations in the ionization process within a substance. In the case of xenon this value is $0.15 \pm 0.02[41]$. The ultimate resolution of a detector using this process to measure energy depositions can be calculated by the following equation [41]:

$$
\sigma=2.35 \sqrt{\frac{F w}{E}}
$$

where $w$ is the energy required to ionize the gas and $\mathrm{E}$ is the total energy deposited. Thus, the ultimate energy resolution such a detector could achieve for $1 \mathrm{MeV}$ of energy deposited is $2.35 \sqrt{(0.15)(22 \mathrm{eV}) /(1 \mathrm{MeV})}=0.4 \%$.

\subsection{Cesium iodide photocathodes}

CsI can be used to create a photocathode which is sensitive in the ultra-violet range, with the highest quantum efficiencies being demonstrated for VUV photons at $\sim 170$

nm. A CsI photocathode operates under the principle of the photoelectric effect, where incoming photons can eject electrons from the bulk of the CsI. One technique for producing these photocathodes is to deposit CsI powder onto a metal substrate using vacuum evaporation. It has been shown that CsI photocathodes can have a quantum efficiency (QE, the average number of electrons ejected by an incident photon) for $170 \mathrm{~nm}$ photons of around $20 \%$, for photocathodes produced in this manner [42]. The quantum efficiency rapidly drops with increasing wavelength though, and is essentially 
zero above $\sim 220 \mathrm{~nm}[43]$.

\subsubsection{Maximizing quantum efficiency}

There are several factors which affect the QE of these photocathodes. Anderson, Kwan, and Peskov state that these factors include the purity of CsI used in the production of the photocathode, the application method of the CsI, and the postproduction treatment of the photocathode [44]. They also state that the environment the photocathode is run in is important, stating that the QE in methane can be as much as $80 \%$ higher than that measured in vacuum. CsI powder is hygroscopic and also absorbs hydrocarbons from air, so it is important that new photocathodes have minimal air exposure. Anderson et. al. have measured a range of QEs that varies by a factor of 2 [42] for photocathodes produced using the same procedure. Heating the photocathode in vacuum both during and after CsI deposition has the effect of improving the QE. It has been observed that the QE of a CsI photocathode operated in methane with gas gain can increase by as much as $20 \%$ as it is illuminated with VUV photons. Cusanno et al. show that the thickness of the CsI deposition also has an effect on the quantum efficiency which becomes maximal when the thickness is $\sim 100-250 \mathrm{~nm}[45]$. It is therefore important that the CsI coating on a photocathode have a uniform thickness as variations in the $\mathrm{QE}$ will adversely affect the energy resolution of the photocathode.

\subsubsection{CsI photocathode response in methane}

Not only does a gas like methane help improve the quantum efficiency of a CsI photocathode, but it also allows one to achieve substantial electron multiplication. This can be of benefit when attempting to measure small signals (i.e. when dealing with low initial numbers of photoelectrons), or when large electronic noise is present. With 
a strong enough electric field, a drifting electron can ionize the $\mathrm{CH}_{4}$, and any freed electron can do the same. The effect is such that more and more free electrons are produced over the drift distance; this is known as an electron 'avalanche', and is shown schematically in Figure 3.1.

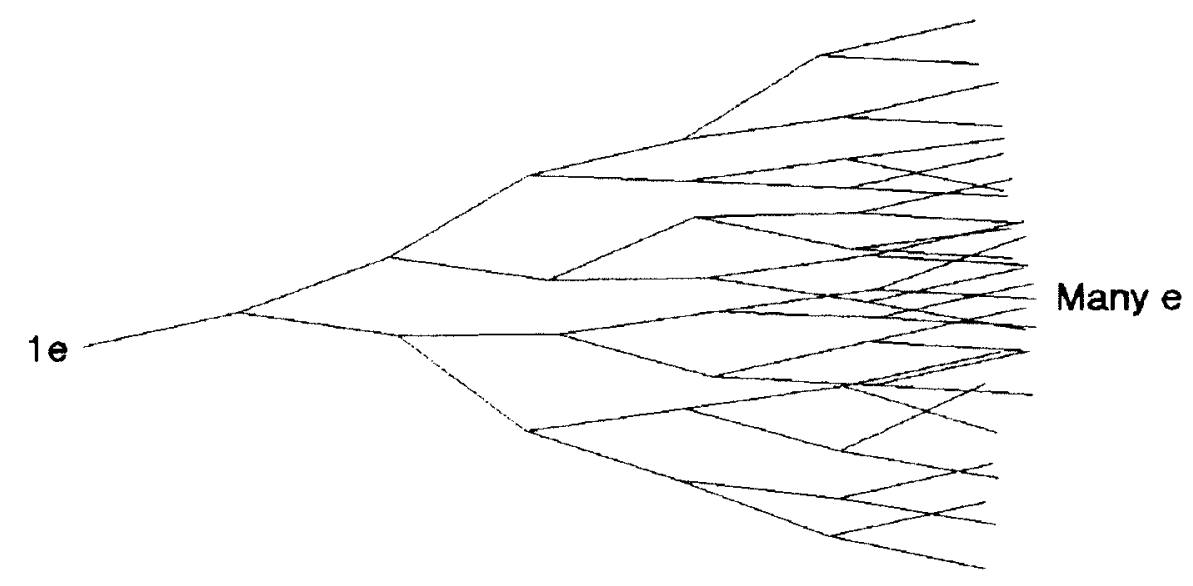

Figure 3.1: A schematic of an electron avalanche, with the lines representing the trajectories of electrons moving to the right. A single electron moving left to right can free a second electron which can go on to free another, which in turn can free yet another, and so on. The effect is an 'avalanche' of free electrons and corresponding production of positive ions.

The signals that are read out during an avalanche are from both the ionization electrons drifting to the mesh and the positive ions drifting back to the photocathode. This is in contrast to the situation where no gain is applied (no avalanche occurs) and the signal is produced as the electron drifts away from the photocathode. 


\section{Chapter 4}

\section{The XEP Time Projection Chamber and Detector Packages}

\subsection{Introduction}

The Carleton University contingent of the EXO collaboration is building a prototype detector to demonstrate the feasibility of using gaseous xenon to search for the neutrinoless double beta decay of ${ }^{136} \mathrm{Xe}$. The goals of XEP are to show that an energy resolution of less than $1 \%$ is possible, as well as to show improved track reconstruction and position resolution over a LXe detector. An improvement in position resolution corresponds to improved background rejection. The XEP time projection chamber is constructed in a clean room and is surrounded by either steel or water shielding; a drawing of this is shown in Figure 4.1. XEP can be divided into three main components which will be described in greater detail: the drift region, the two detector packages, and the two endcap regions. The TPC is housed in a stainless steel pres-

sure vessel. This vessel is necessary to withstand the force exerted by the Xe at the designed operating pressure of 10 bar. This 10 bar of xenon will be present in the drift region and the end cap regions. 


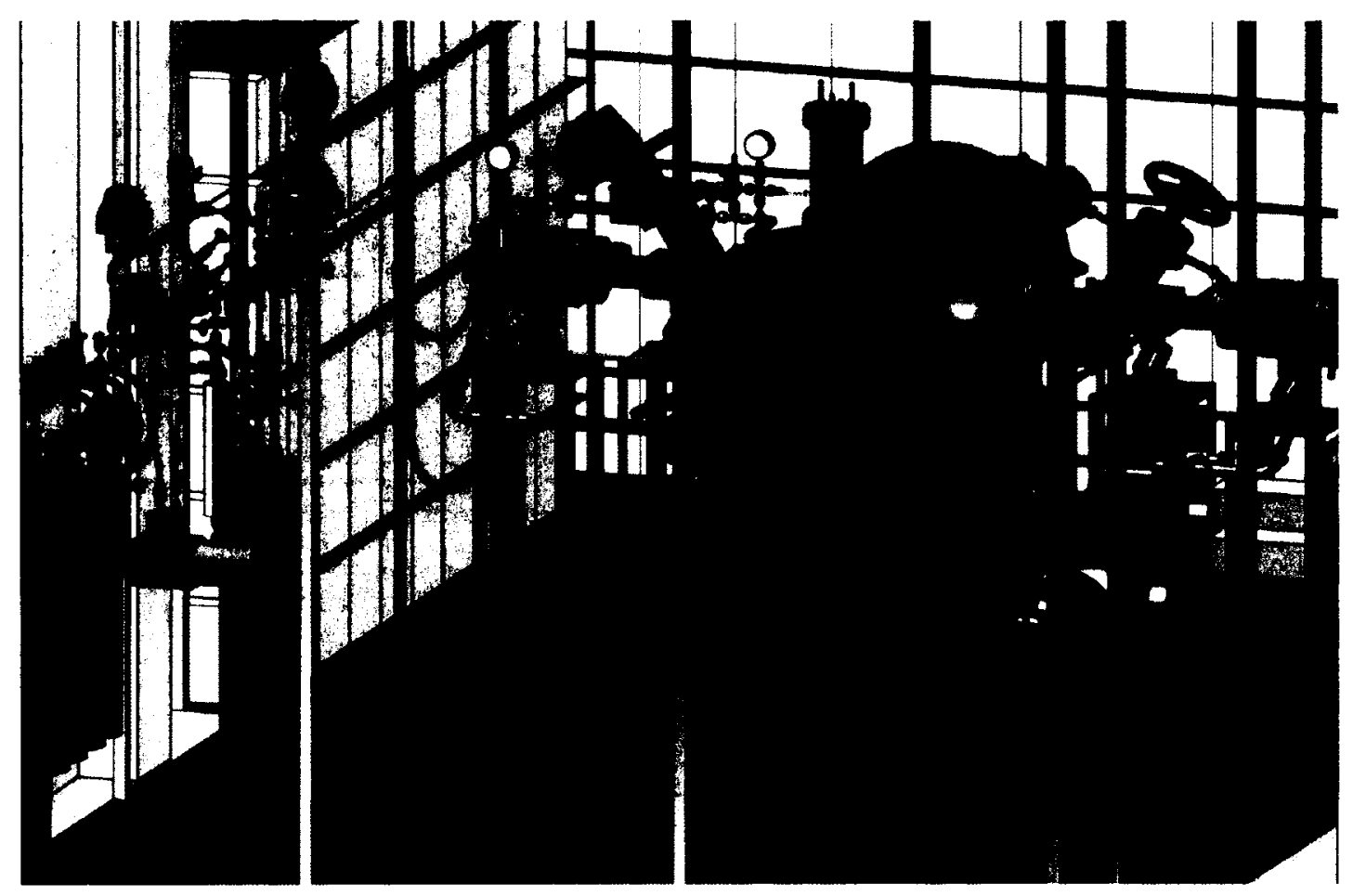

Figure 4.1: A drawing of the XEP cleanroom and TPC at Carleton University. To the left are the gas process systems. The TPC sits over three layers of 1 inch thick steel plates. The steel plates and water-filled boxes around the outside provide shielding from gammas emitted from the concrete walls.

The end cap regions are the bell-shaped regions shown in Figure 4.2 at the ends of the TPC. They are connected to each other by external gas plumbing. The purpose of having gas in this region is to minimize any differential pressure across the fragile detector package. The pressure between the end caps and the drift region will be monitored by a differential pressure gauge. If the differential pressure rises above 70 mbar there is danger of breaking the window and printed circuit board (PCB) of the detector package (see Section 4.3 for a description of these components). In the event that a 70 mbar or higher pressure differential is present, a valve connecting the two regions will open equalizing the pressure. 


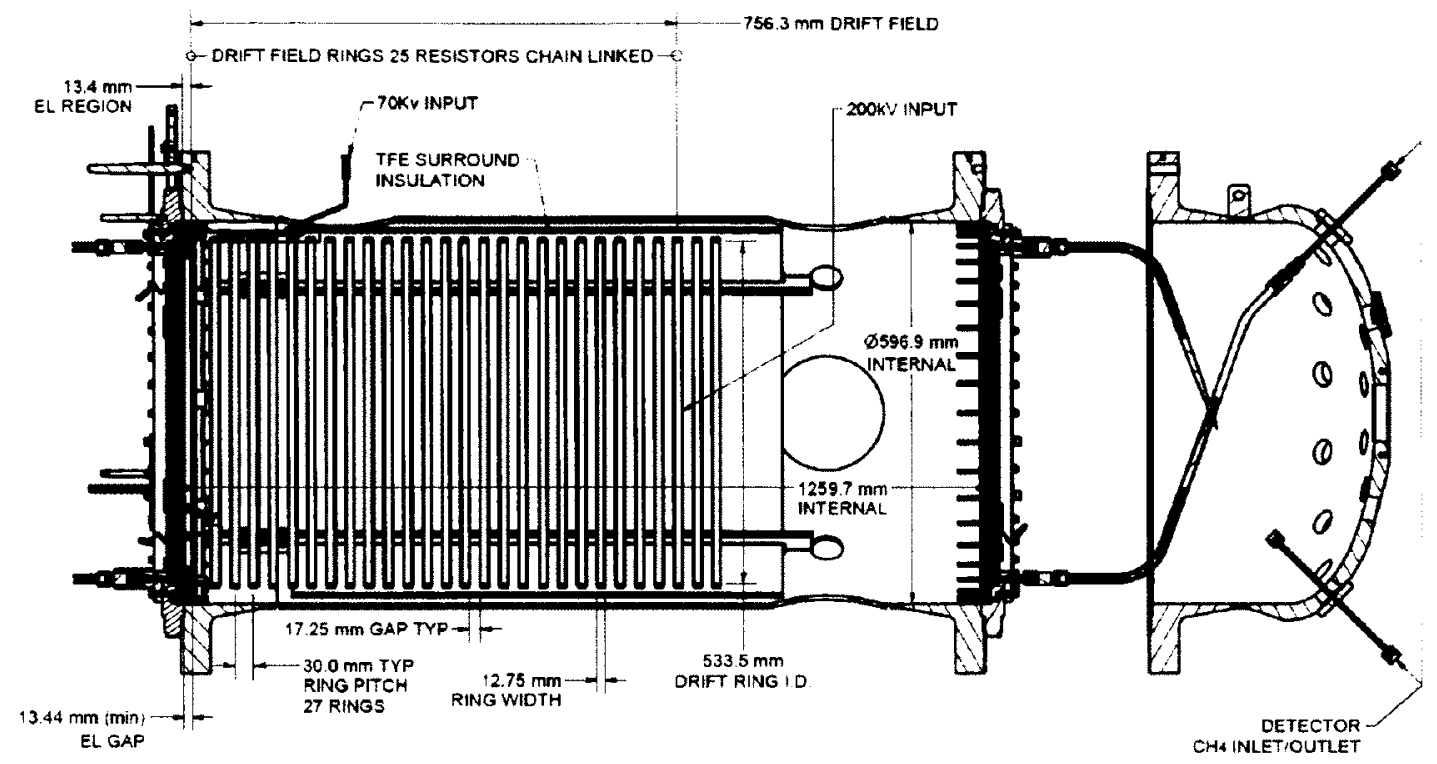

Figure 4.2: Cross section of the XEP time projection chamber. The end cap for the left side is identical to that on the right, but is not shown.

\subsection{The drift region}

The drift region is a cylindrical region $76 \mathrm{~cm}$ long and $53 \mathrm{~cm}$ in diameter. This region is contained in the larger central volume of the cylindrical pressure vessel which will have a detector package at either end. The window-to-window separation is $1.26 \mathrm{~m}$, and the inner diameter is $60 \mathrm{~cm}$. The layout of the drift region within the TPC is show in Figure 4.2. The outer wall of the drift region is lined with $\sim 4 \mathrm{~mm}$ Teflon which will electrically insulate the field rings from the wall of the pressure vessel and will also reflect VUV light. Inside this Teflon lining is a series of copper field shaping rings generating an electric field of up to $0.8 \mathrm{kV} \cdot \mathrm{cm}^{-1} \cdot \mathrm{bar}^{-1}$. Since the EL threshold is $0.83 \mathrm{kV} \cdot \mathrm{cm}^{-1} \cdot \mathrm{bar}^{-1}$, this region will not produce any EL light. It will contain roughly $170 \mathrm{~L}$ of $\mathrm{Xe}, \sim 10 \mathrm{~kg}$, at a pressure of $10 \mathrm{bar}$. The full volume window-to-window is roughly $350 \mathrm{~L}$, containing $\sim 20 \mathrm{~kg}$ of Xe at 10 bar. 


\subsubsection{Electroluminescence (EL) region}

The EL region, shown in Figure 4.3, is a $13.44 \mathrm{~mm}$ gap at the lower potential end of the field shaping rings. This region is defined by two wire planes with one plane grounded and the other at a maximum potential of $70 \mathrm{kV}$. The planes are isolated spatially and electrically by a Teflon spacer. The wires of these planes are anchored in a circular stainless steel frame with an inner diameter of about $500 \mathrm{~mm}$. The 189 $0.254 \mathrm{~mm}$ thick stainless steel wires are strung parallel to each other with a pitch of $2.5 \mathrm{~mm}$. The EL region is designed to operate at a maximum electric field of 7 $\mathrm{kV} \cdot \mathrm{cm}^{-1} \cdot \mathrm{bar}^{-1}$, though typical operation will be at lower fields. From Eq. 3.5 the EL region will generate roughly 240 photons from an ionization electron drifting in $10 \mathrm{bar} \mathrm{Xe}$ in an electric field of $1 \mathrm{kV} \cdot \mathrm{cm}^{-1} \cdot \mathrm{bar}^{-1}$.

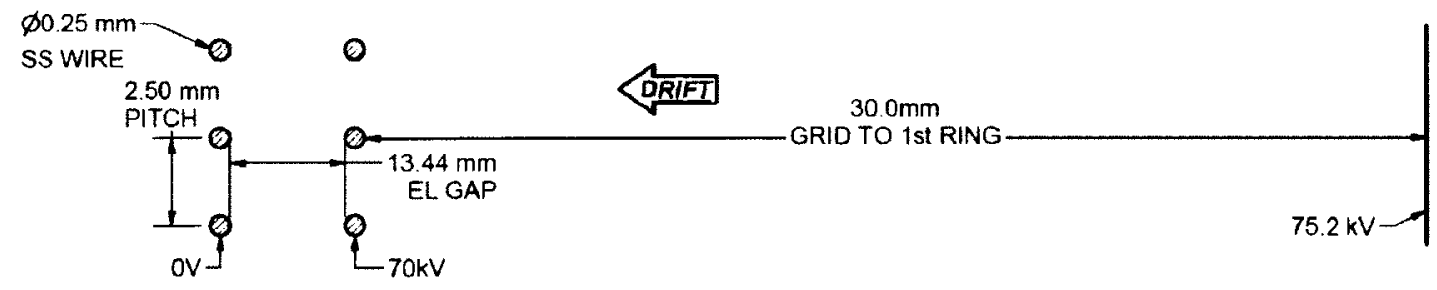

Figure 4.3: A section of the EL grid including the last field shaping ring in the chain.

\subsection{The detector packages}

XEP's detector packages will be used to measure the scintillation and EL light and produce a measurable electrical signal. Two detector packages will be mounted on the TPC, with either package between the drift region and an end cap region. Each detector package is a sandwich of a fused silica window, nickel mesh, Teflon spacer, and CsI photocathode. Two cross-sections of the detector package are shown: Figure 4.4 shows a technical drawing of all the components, and Figure 4.5 shows a simplified version including a description of signal generation from the EL region. 


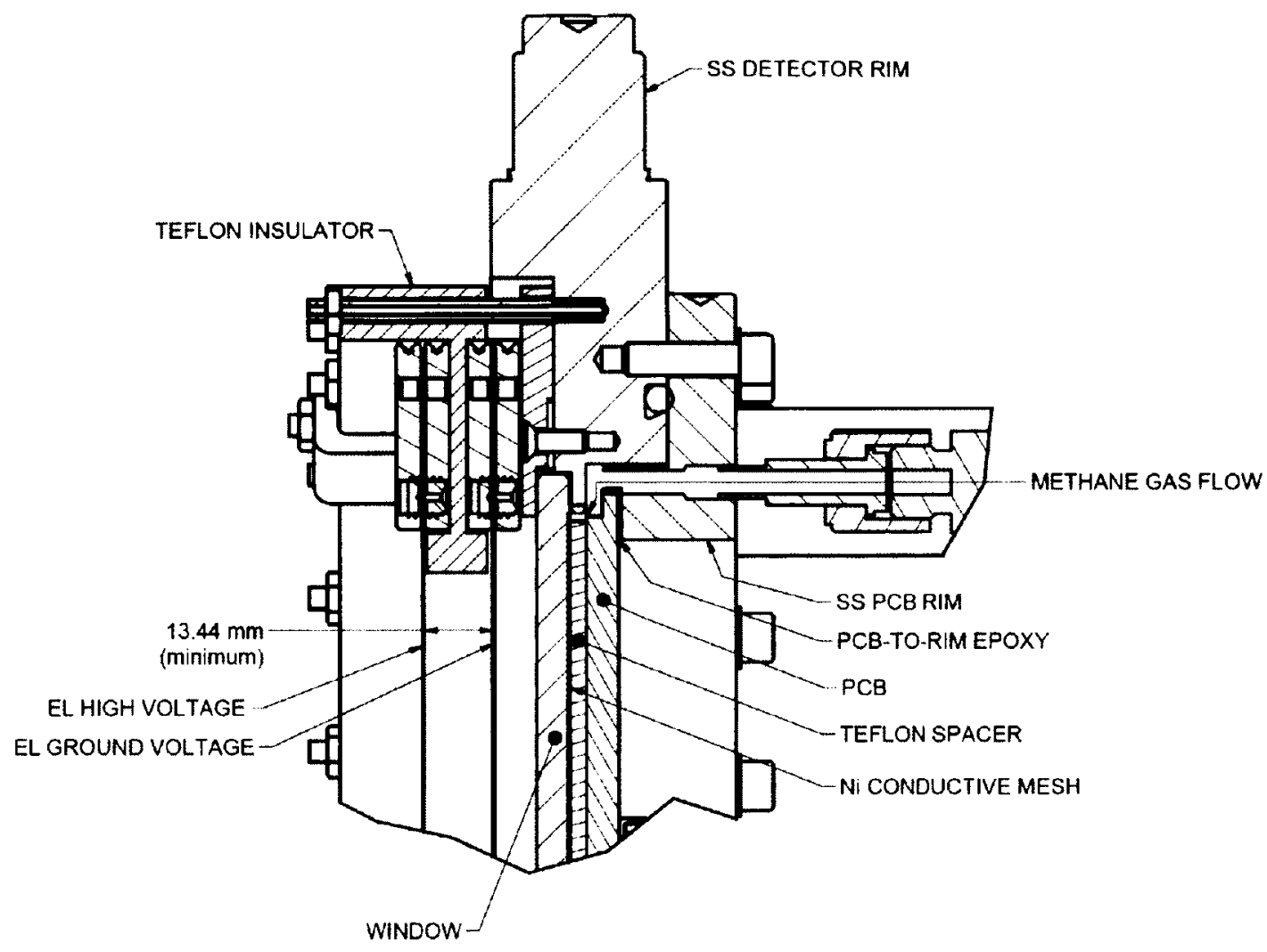

Figure 4.4: A cross section of the XEP detector package. 


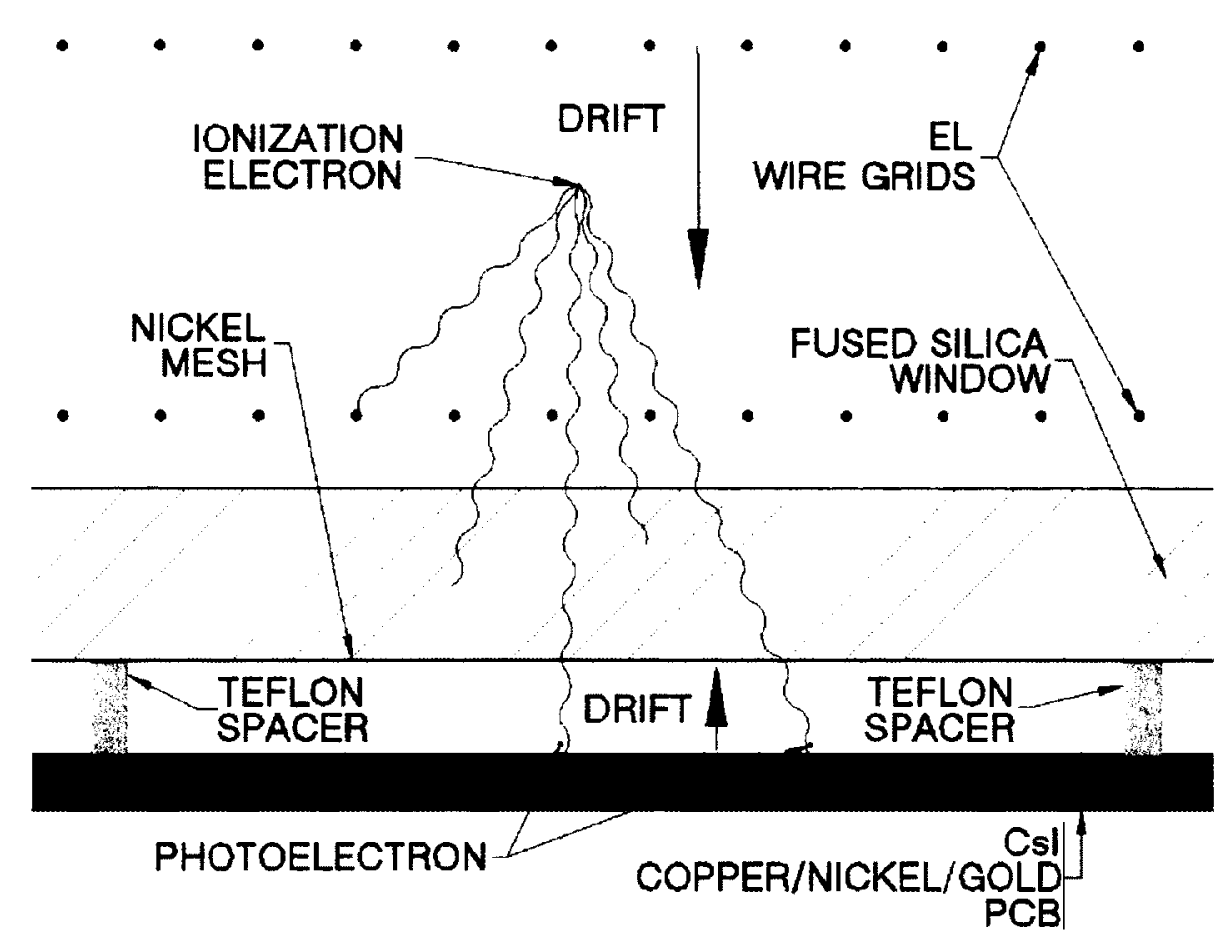

Figure 4.5: Schematic of the detector package showing how signals are generated. An ionization electrons will drift to the region defined by the EL wire grids where it will gain enough energy to generate secondary scintillation photons. This schematic only shows photons travelling toward the photocathode, though the true distribution of photon generation is isotropic. Photons which pass through the wire grid and fused silica window and strike the CsI surface of the photocathode can produce photoelectrons. These photoelectrons will drift upwards to the nickel mesh. If methane is present between the nickel mesh and the PCB, and a large enough electric field is applied, the photoelectrons will ionize the methane, producing clouds of electrons in an electron avalanche.

\subsubsection{Fused silica window}

The window component of the detector package separates the high pressure Xe of the drift and EL regions from the low pressure $\mathrm{CH}_{4}$ region. Fused silica was chosen because of its relatively good transmission of $172 \mathrm{~nm} \mathrm{UV}$, though this is dependent on the grade of material. The exact material chosen is an unpolished piece of Corning HPFS 7980-OA. This material has a transmission coefficient for $172 \mathrm{~nm}$ photons of 
$\sim 50 \%$. The window, with a diameter of $520.7 \mathrm{~mm}$ and a thickness of $6.35 \mathrm{~mm}$, is epoxied to the steel frame of the detector package. If one considers a window with a $172 \mathrm{~nm}$ transmission coefficient of $50 \%$, a photocathode with a quantum efficiency of $20 \%$, and a solid angle correction factor of 0.2 for the centre of the EL region, then the 240 photons produced from a single ionization electron will liberate roughly $240 \times 0.5$ x $0.2 \times 0.15=4$ electrons from the photocathode. A $1 \mathrm{MeV}$ primary electron which would produce 45,000 ionization electrons will then correspond to 180,000 electrons produced at the photocathode.

\subsection{2 $\mathrm{CH}_{4}$ region and $\mathrm{CsI}$ readout pads}

The VUV signal readout of the detector is achieved through an array of square photocathode pads. Each pad is $25.4 \mathrm{~mm}$ on edge and is positioned with a $1 \mathrm{~mm}$ gap from each adjacent pad. The pads are constructed on a PCB with a $35.6 \mu \mathrm{m}$ thick copper base coated in $7 \mu \mathrm{m}$ of nickel and then $0.5 \mu \mathrm{m}$ of gold. The final layer is a coating of CsI with a thickness on the order of $100 \mathrm{~nm}$. The purpose of the nickel layer is to provide a physical boundary between the copper and the CsI. This region spans $3.175 \mathrm{~mm}$ defined by a Teflon spacer between the pads and a nickel mesh at the window. The spacer is machined with square openings which expose the CsI readout pads to the EL light. A drawing of the board is shown in Figure 4.6.

The nickel mesh is made from a thin sheet of nickel and has $106 \mu \mathrm{m}$ holes at a 127 $\mu \mathrm{m}$ pitch. The mesh has a transmission coefficient of $70 \%$. The pads on the board are held at ground while a positive voltage is applied to the nickel mesh to drift and collect electrons. There are two gas plumbing connections to this part of the detector package (see Figure 4.2). With these two connections, $\mathrm{CH}_{4}$ will be flowed and purified. To generate measurable signals this region will contain low pressure (25 to $130 \mathrm{mbar}$ ) $\mathrm{CH}_{4}$. For ease of comparison to the primary literature regarding CsI photocathodes, the $\mathrm{CH}_{4}$ pressures will henceforth be stated in units of Torr, rather than mbar. The 
previously stated range is equal to roughly 20 to 100 Torr. This $\mathrm{CH}_{4}$ will allow for the multiplication of the photoelectrons ejected from the photocathode as they drift toward the nickel mesh. Considering the signal to charge a $1 \mathrm{pF}$ capacitor, the 180,000 photoelectrons produced at the photocathode for a $1 \mathrm{MeV}$ primary electron will generate a signal of $180,000 \times 1.602 \times 10^{-19} \mathrm{C} / 10^{-12} \mathrm{~F} \approx 30 \mathrm{mV}$. Thus, when gain is present, signals from XEP will be easily measurable.

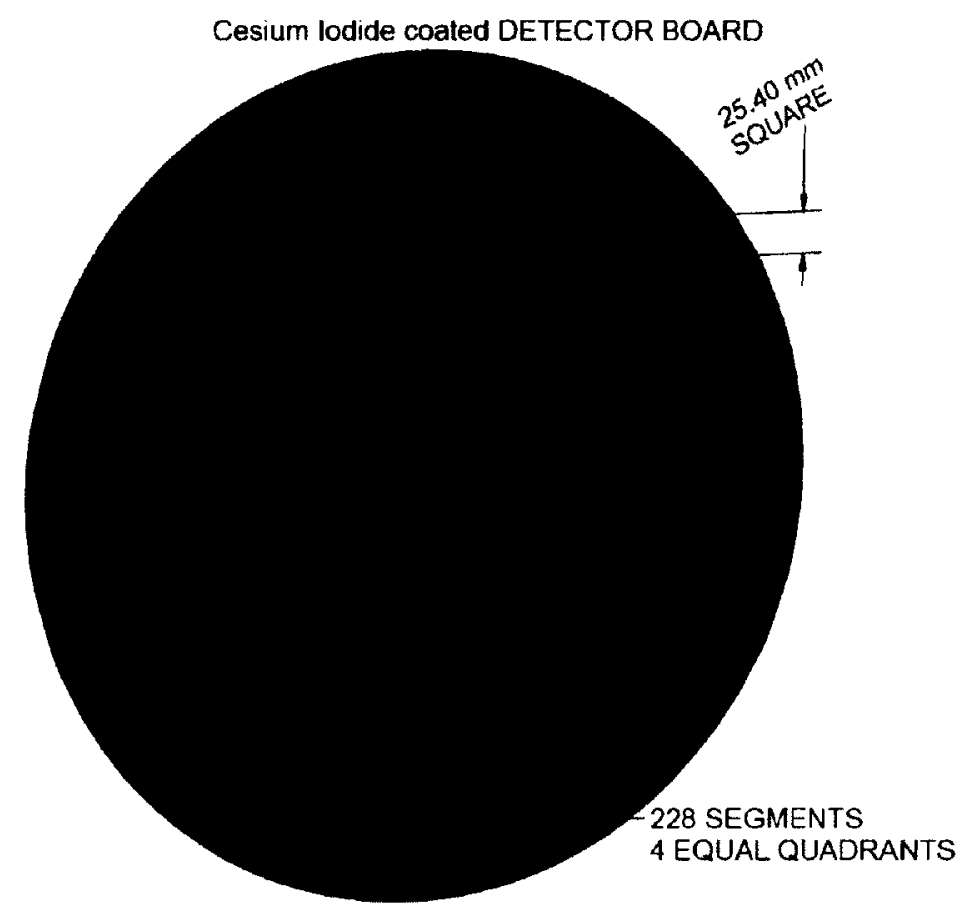

Figure 4.6: The CsI readout board showing all 228 pads.

\subsection{Initial detector package}

The detector package described previously is the proposed final state package. During the initial commissioning of XEP a simplified version was used. The Teflon spacer in this package does not contain cut outs for all of the readout pads, but rather has an open $4 \times 4$ grid centred on the photocathode, as also exposes 2 outer pads in each 
quadrant. This preliminary spacer is shown in Figure 4.7.

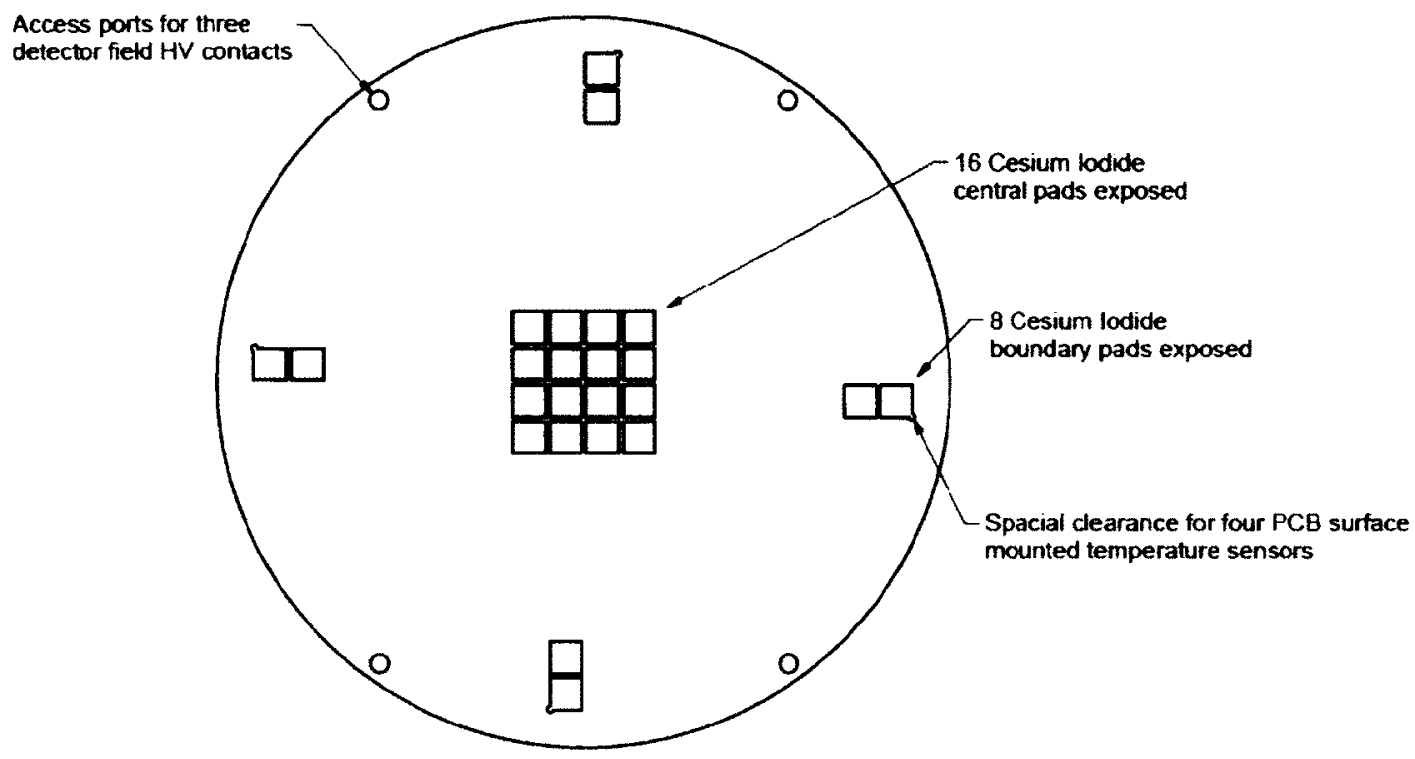

Figure 4.7: Drawing of the Teflon spacer to be used with the initial detector package.

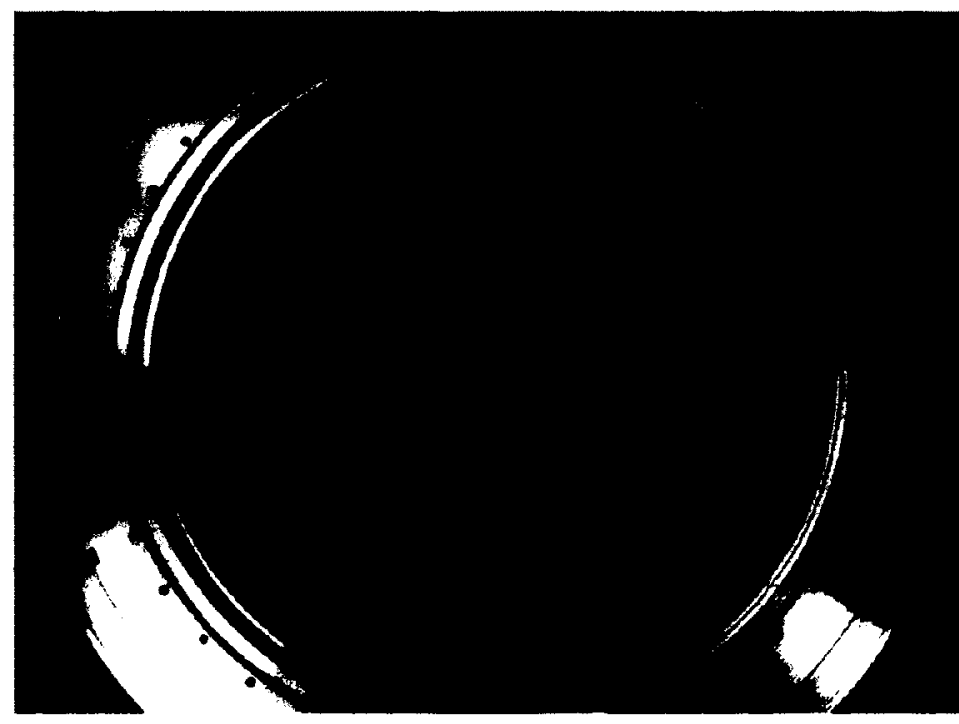

Figure 4.8: Detector package ring with window, mesh, and Teflon in place. Ripples in the nickel mesh are clearly visible; these were extremely difficult to avoid as the mesh is so fine. The locations of the pads which were examined for this thesis (Chapter 6) are labelled.

Figure 4.8 shows the steel frame of the detector package containing the fused silica 
window, the nickel mesh, and the Teflon spacer; ripples in the mesh are obvious here. The mesh is very fine and consequently is not very rigid. Because of this lack of rigidity the mesh was very hard to place in a smooth layer. The next version of the detector package will include a more rigid mesh. The CsI photocathode is shown in Figure 4.9. This image was taken after the vacuum evaporation of CsI onto the board, immediately before it was mounted to the steel frame.

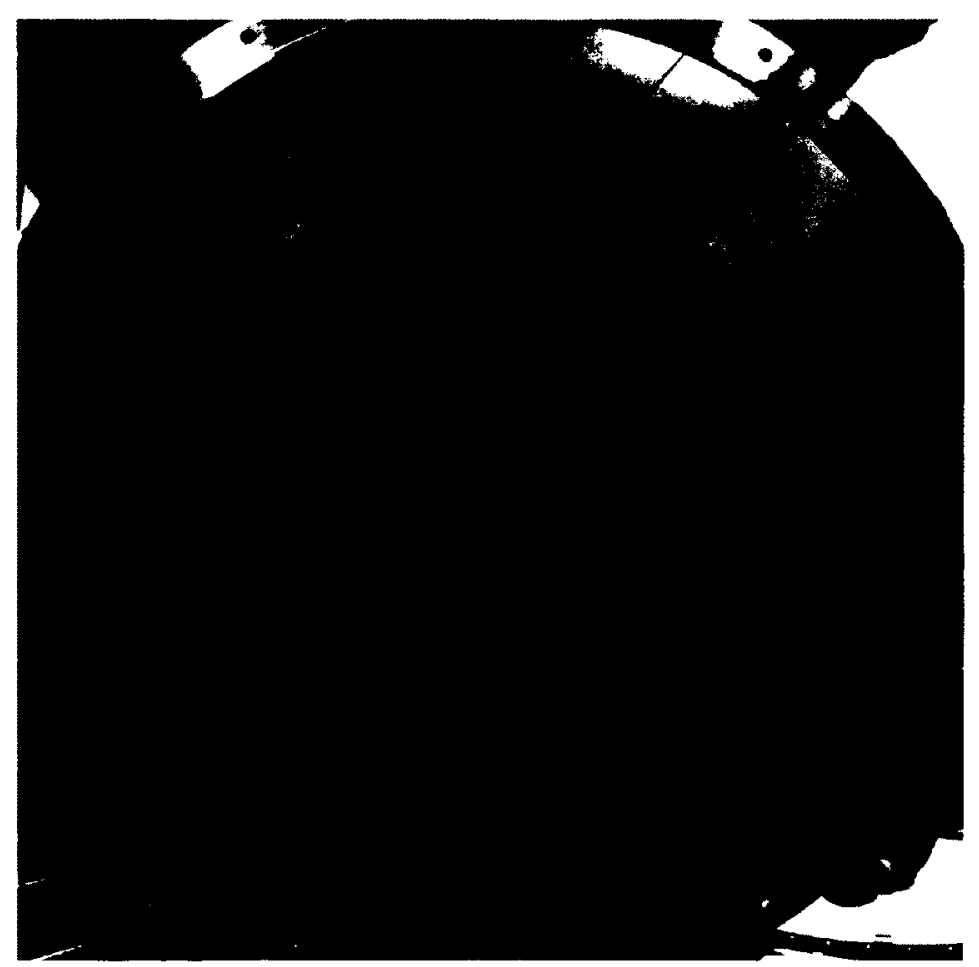

Figure 4.9: The CsI readout board is shown here with the first deposition of CsI. The main target of the deposition was the central area spanning $4 \times 4$ pads. Most of the rest of the board was covered with aluminum foil during the deposition, though the uncovered areas are obvious. 


\section{Chapter 5}

\section{Secondary XEP Equipment}

\subsection{Data acquisition electronics}

The XEP data acquisition (DAQ) system consists of 2 CAEN SY2791 TPC Readout System racks. Each $\mathbf{S Y 2 7 9 1}$ contains $1 \mathbf{A 2 7 9 3}$ power supply unit and up to 8 A2792 acquisition modules which are capable of processing up to 32 channels each. In total, the system consists of 512 individual readout channels. Each A2792 module contains 16 custom preamplifier chips which plug into the board. Each preamplifier chip handles 2 channels; the properties of the preamplifiers used are shown in Table 5.1. The preamplifier outputs are digitized by 12 bit $2.5 \mathrm{MSample} / \mathrm{s}$ flash analog-todigital converters $(A D C)$, processed by the internal acquisition logic, implemented in a field programmable gate array (FPGA), and stored in circular memory buffers before being readout via fibre optic to a $\boldsymbol{A 2 8 1 8}$ PCI card housed in a PC. Each module contains two digital-to-analog converters (DACs), one for channels 1-16 and one for channels 17-32, which can be used to add a DC offset to the signal after it has passed through the shaping filters. The output of the DACs can be set individually. Each channel has $1 \mathrm{MB}$ of memory which is evenly divided between the circular buffers. We have labelled the two A2793 power supply units 'A' and 'B', and the 8 A2792 modules for each A2793 'A0' ('B0') through 'A7' ('B7'). 


\begin{tabular}{cc}
\hline Property & Value \\
\hline \hline FET integrator time constant & $470 \mu \mathrm{s}$ \\
Sensitivity & $11.5 \mathrm{mV} / \mathrm{fC}$ \\
Shaper integration time constant & $0.6 \mu \mathrm{s}$ \\
Shaper differentiation time constant & $2 \mu \mathrm{s}$ \\
Signal to noise at $1 \mathrm{fC}(\mathrm{C}=200 \mathrm{pF})$ & 10 \\
\hline
\end{tabular}

Table 5.1: Properties of the preamplifiers used in the CAEN data acquisition system.

For the initial commissioning of XEP using the initial detector package a sub-set of the full CAEN system was used. This set consisted of the 'B' A2793 power supply, and 4 of the A2792 modules, B0, B1, B2, and B3.

\subsubsection{Characterization of noise in the CAEN data acquisition system}

The tests performed to characterize the CAEN digitizers included measurements of their baseline when triggered by a signal from a pulse generator and measurements of variations in the pulse heights. The generator used was a BNC Model PB-4 Pulse Generator with the following settings: Amplitude $(131.9 \mathrm{mV})$, Delay $(10 \mu \mathrm{s})$, Rise Time $(5 \mu \mathrm{s})$, Fall Time $(200 \mu \mathrm{s})$ for a negative pulse. The pulse generator was connected to both the CAEN A2792 module and the Textronix DPO 5054 oscilloscope. To connect to the the A2792 module's ribbon cable input, a transfer box was made. This box has a lemo input for each channel which connects to the corresponding channel of the ribbon cable through a $1 \mathrm{pF}$ capacitor, and the ribbon cable connects to the the A2792.

Each of the 4 'B' A2792 modules which are to be used with the initial detector package were measured. These were the modules labelled B0, B1, B2, and B3. Each of the 32 channels on each module were measured individually and 5000 pulses were 
recorded. Each channel was programmed with a $13.5 \mathrm{mV}$ DC offset using the internal DAC. The sampling clock was set to $2.5 \mathrm{MHz}$ and each channel was run using 4 memory buffers, with each buffer holding 4096 samples.

A sample waveform is shown in Figure 5.1. A Gaussian was fit to a distribution of the first 1900 pre-pulse samples of each of the 5000 recorded waveforms. Distributions of the mean and width from all of the fits were then each fit by a Gaussian to determine the baseline mean and standard deviation for each channel. A sample of one of these distributions is shown in Figure 5.2. These fit values are shown together in Figures 5.5, 5.7, 5.9, 5.11 for modules B0, B1, B2, and B3, respectively. Next, the baseline was subtracted from the height of the pulse for each of the 5000 pulses. A Gaussian was fit to a distribution of these pulse heights. Distributions of the mean and width from all of the fits were then also each fit by a Gaussian to determine the pulse height mean and standard deviations. A sample of one of these distributions is shown in Figure 5.3. These fit values for each channel are shown together in Figures 5.4, 5.6, $5.8,5.10$ for modules B0, B1, B2, and B3, respectively. 

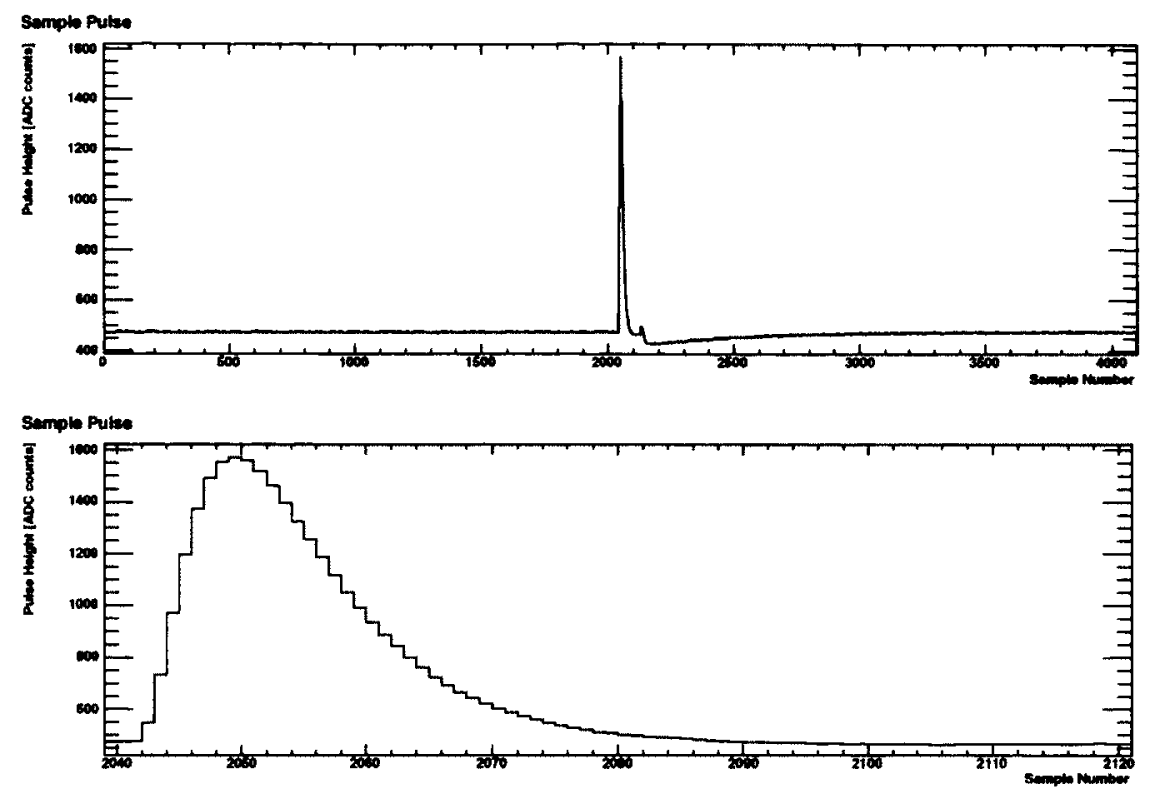

Figure 5.1: A sample waveform measure by the CAEN data acquisition electronics. The pulse height is taken as the maximum value of the waveform. The upper plot shows all 4096 samples in the trace, while the lower plot shows the same waveform focused around the actual pulse.

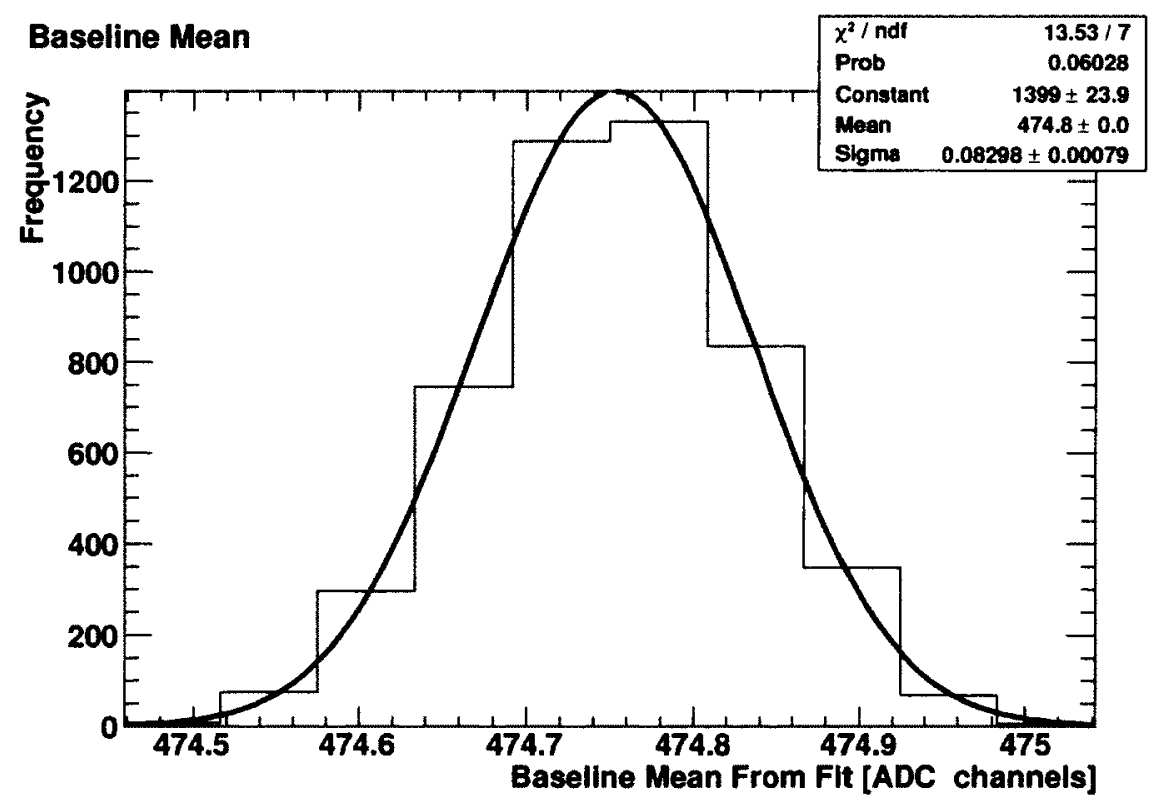

Figure 5.2: A sample distribution of measured baseline means in CAEN module B0, channel 29 . 


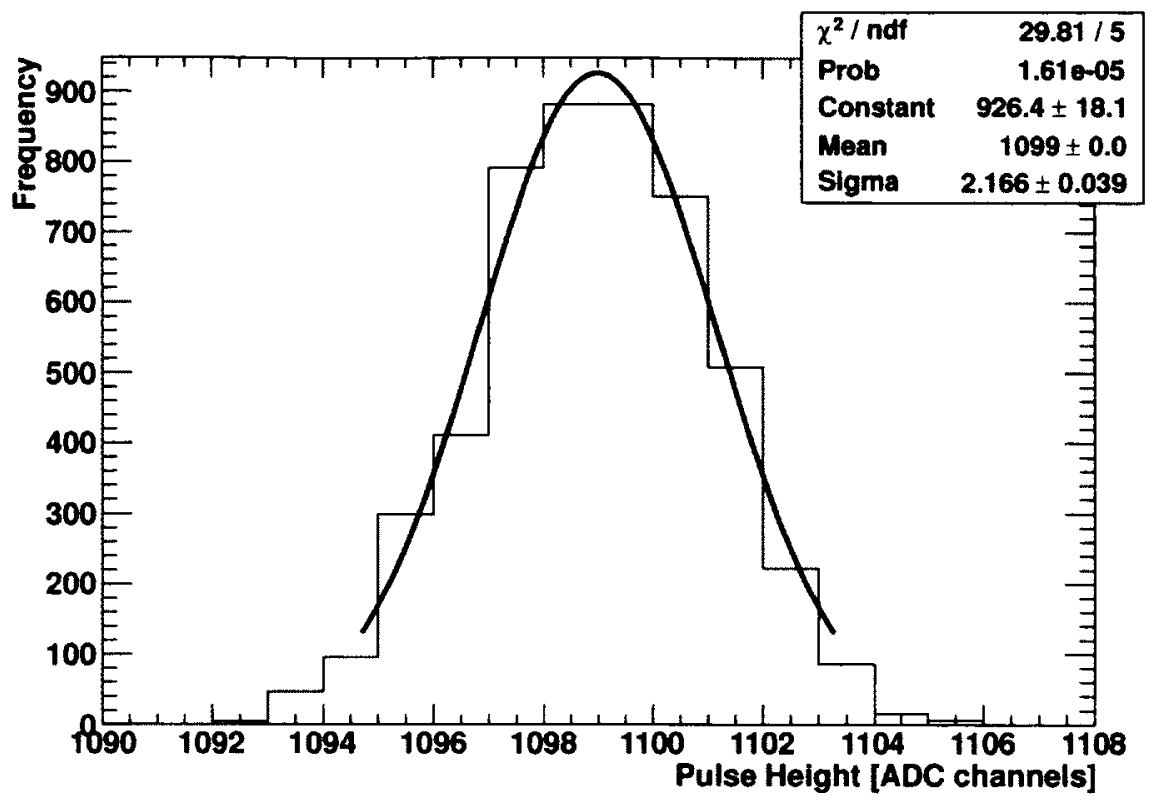

Figure 5.3: A sample distribution of measured pulse heights in CAEN module B0, channel 29.
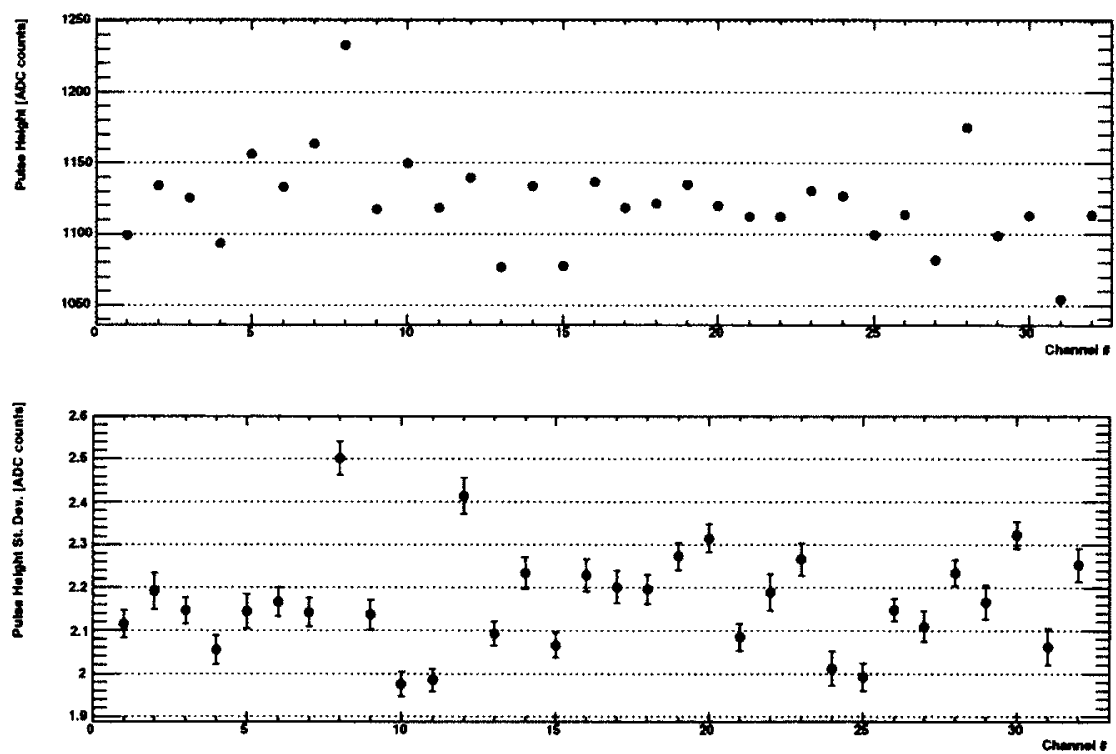

Figure 5.4: Pulse height means and standard deviations for CAEN A2792 module B0. 

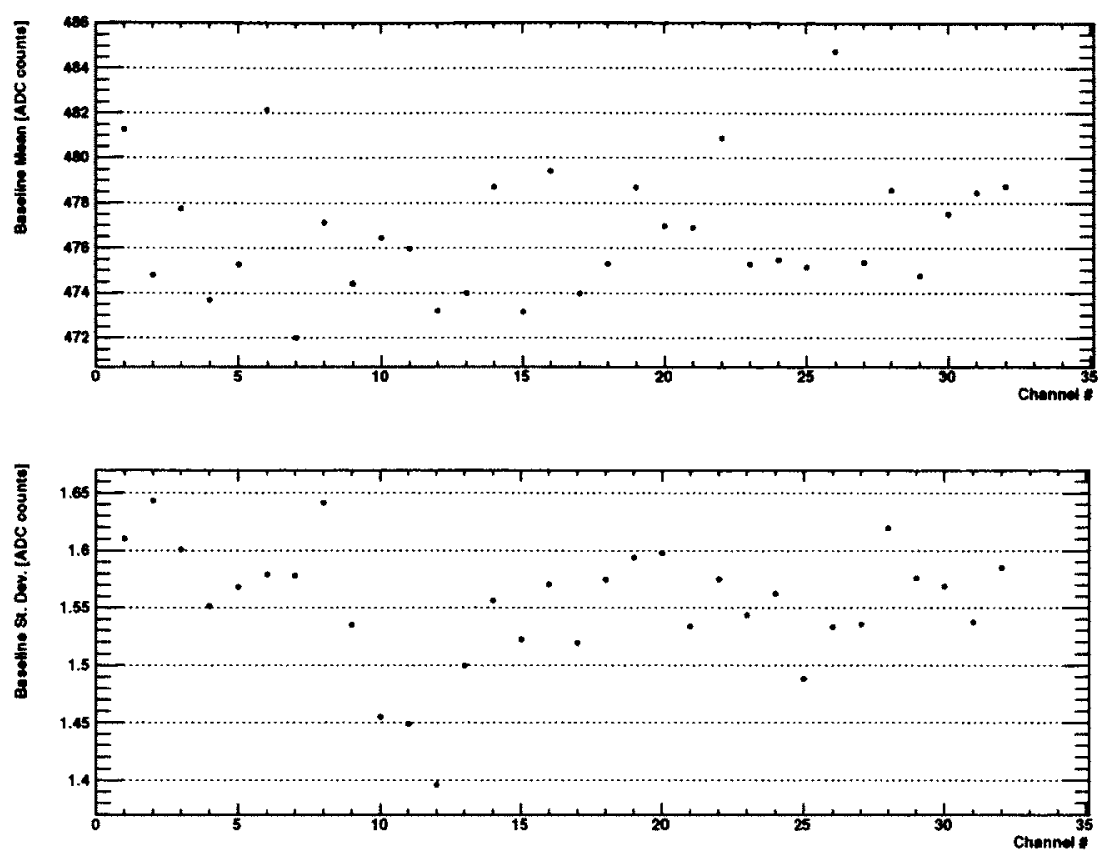

Figure 5.5: The mean and standard deviation of the baseline for each channel of the CAEN A2792 module B0. A DC offset was added using the internal DACs, each set to a value of $13.5 \mathrm{mV}$ (268 channels), before the secondary amplification. 

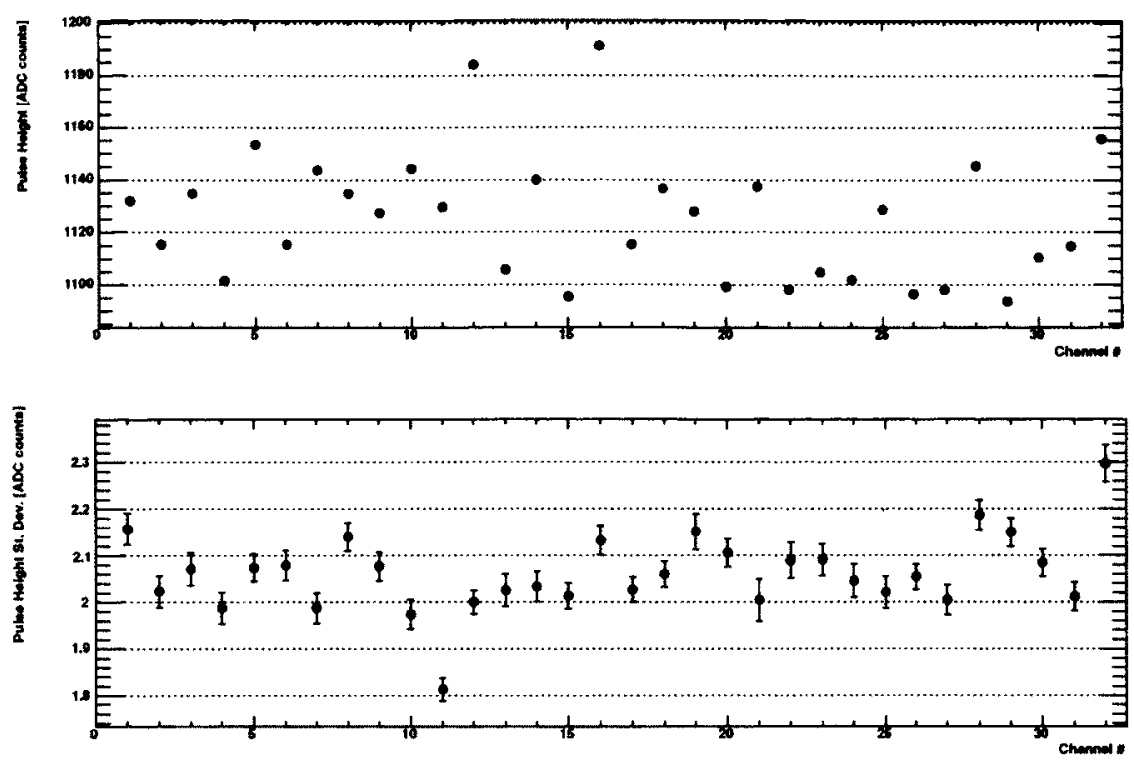

Figure 5.6: Pulse height means and standard deviations for CAEN A2792 module B1.
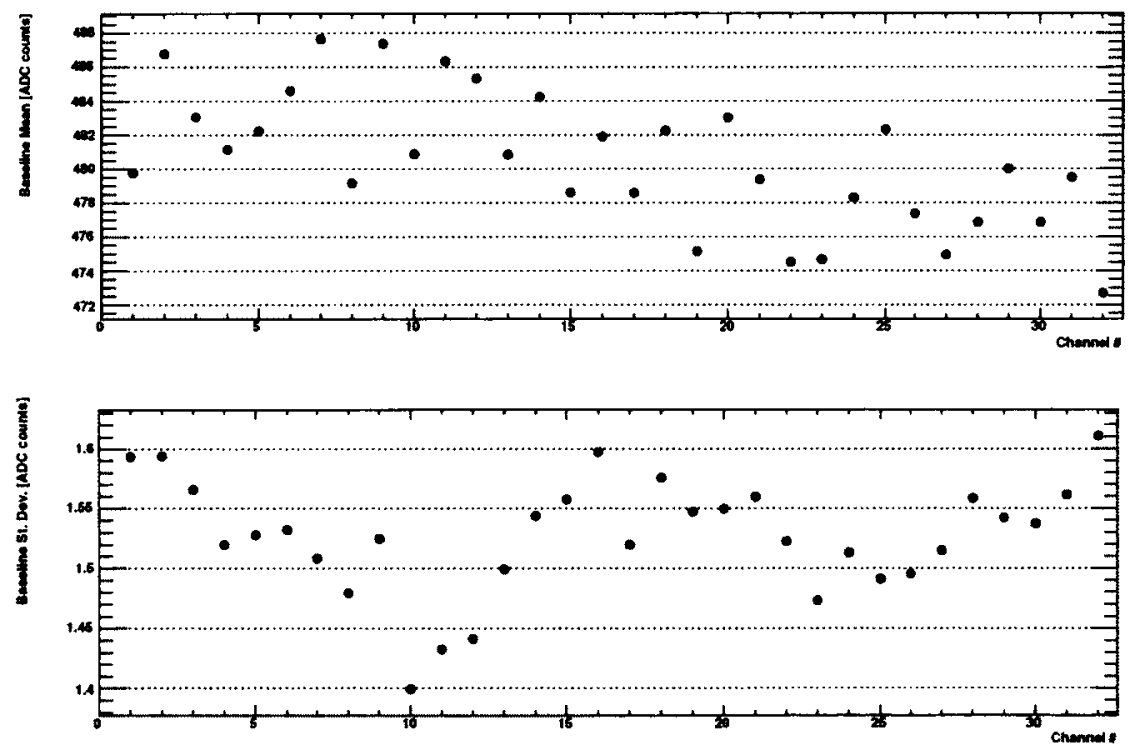

Figure 5.7: The mean and standard deviation of the baseline for each channel of the CAEN A2792 module B1. A DC offset was added using the internal DACs, each set to a value of $13.5 \mathrm{mV}$ (268 channels), before the secondary amplification. 

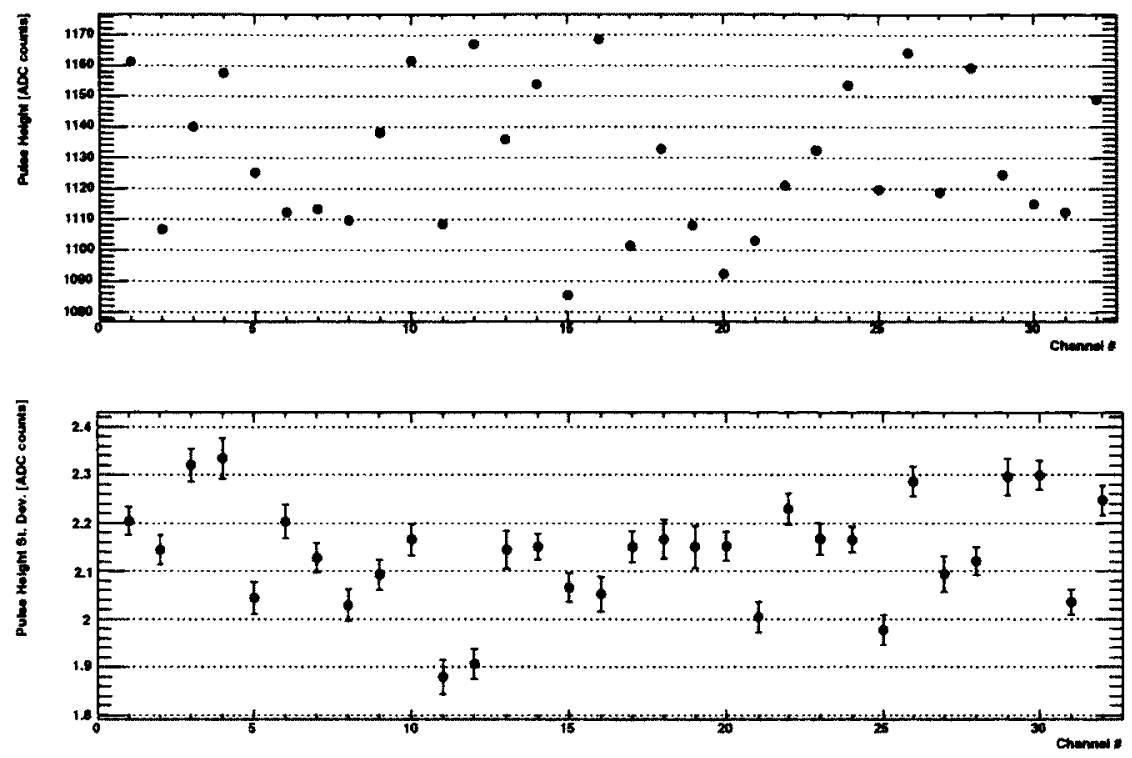

Figure 5.8: Pulse height means and standard deviations for CAEN A2792 module B2.
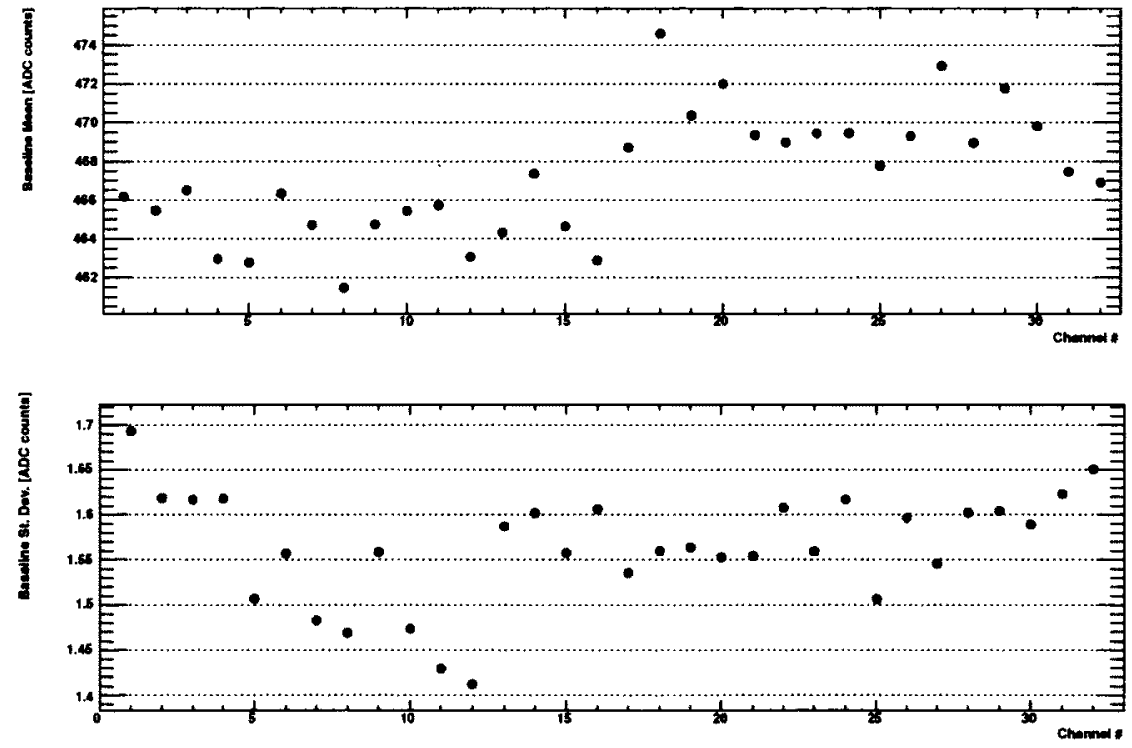

Figure 5.9: The mean and standard deviation of the baseline for each channel of the CAEN A2792 module B2. A DC offset was added using the internal DACs, each set to a value of $13.5 \mathrm{mV}$ (268 channels), before the secondary amplification. 

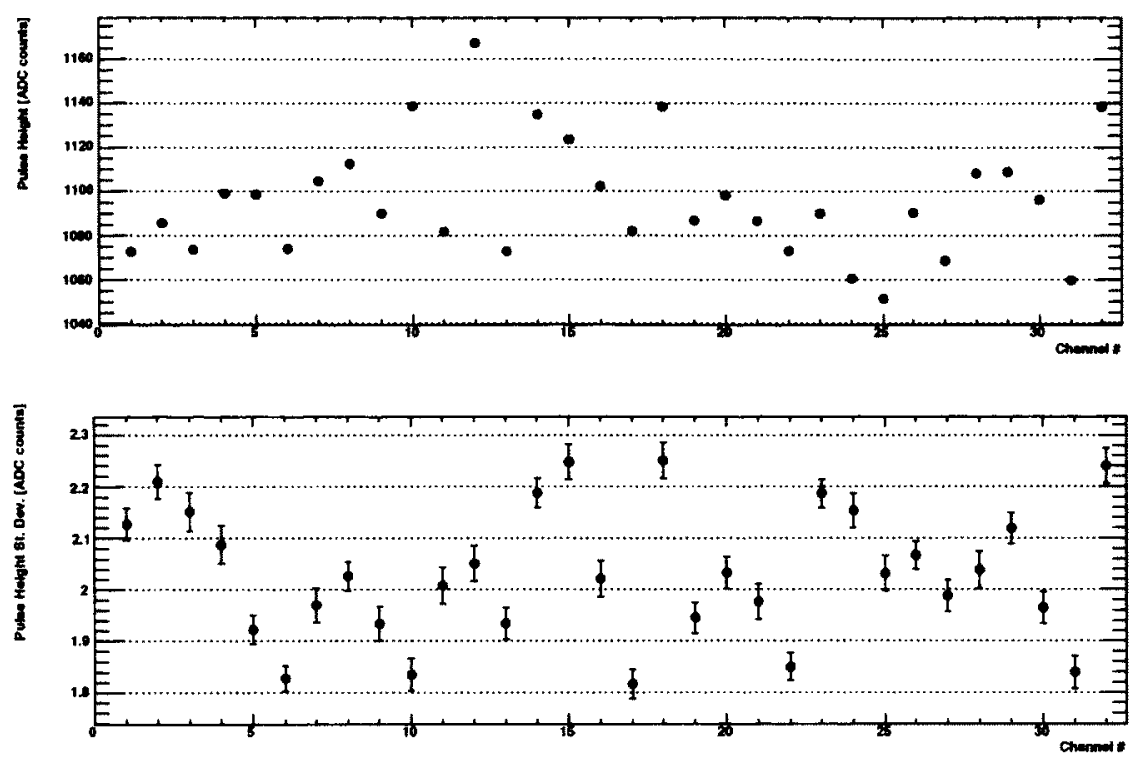

Figure 5.10: Pulse height means and standard deviations for CAEN A2792 module B3.
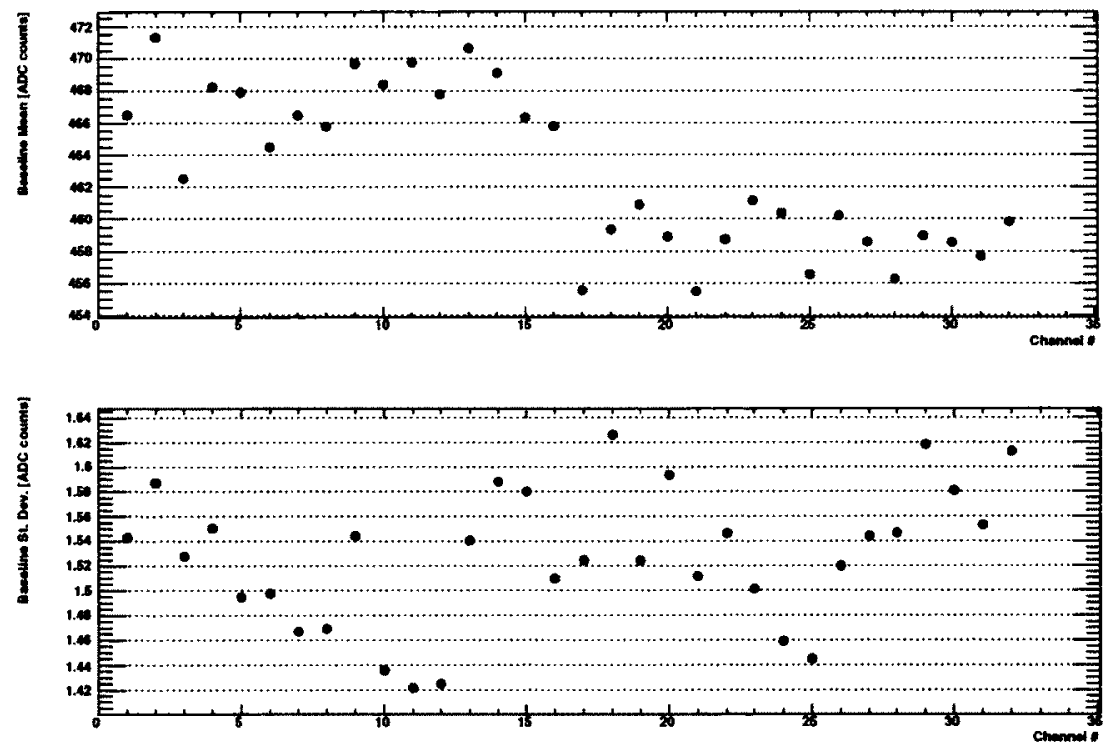

Figure 5.11: The mean and standard deviation of the baseline for each channel of the CAEN A2792 module B3. A DC offset was added using the internal DACs, each set to a value of $13.5 \mathrm{mV}$ (268 channels), before the secondary amplification. 
As seen in the averages of the baseline for each module, there is a difference between the output of the two internal DACs even though they are set to the same offset. This is most noticeable in module B3, shown in Figure 5.11. Apart from this, the baselines are fairly stable with a standard deviation of about 1.5 ADC channels corresponding to $1.2 \mathrm{mV}$ noise.

The variations seen in the pulse heights are slightly higher, around 2.2 ADC channels or $1.8 \mathrm{mV}$. For $1 \mathrm{MeV}$ electron, the electroluminescent yield is producing a signal of roughly $30 \mathrm{mV}$ uniformly distributed over all 228 pads; a single pad may then see a signal of $0.13 \mathrm{mV}$. This is a conservative estimate based on a minimum EL yield. A signal this size cannot be distinguished from the $1.8 \mathrm{mV}$ noise. However, if gain is applied in the methane gap of the detector package, we can reduce the effect of the noise to a negligible level. At a gain of 200 , the total signal summed over all pads becomes $6000 \mathrm{mV}$; at this gain, and assuming uncorrelated noise between channels, the noise is $\sqrt{228}(1.8 \mathrm{mV})=27.2 \mathrm{mV}$, less than $0.5 \%$ of the signal.

Figures 5.12 and 5.13 show the baseline values and pulse heights, respectively, for all 128 channels which were measured. The distribution of pulse heights shows a width of about $2.5 \%$. We will, as expected, have to correct for these differences if we want to achieve XEP's energy resolution goal. Similarly, the baselines show a width of about $1.8 \%$ 


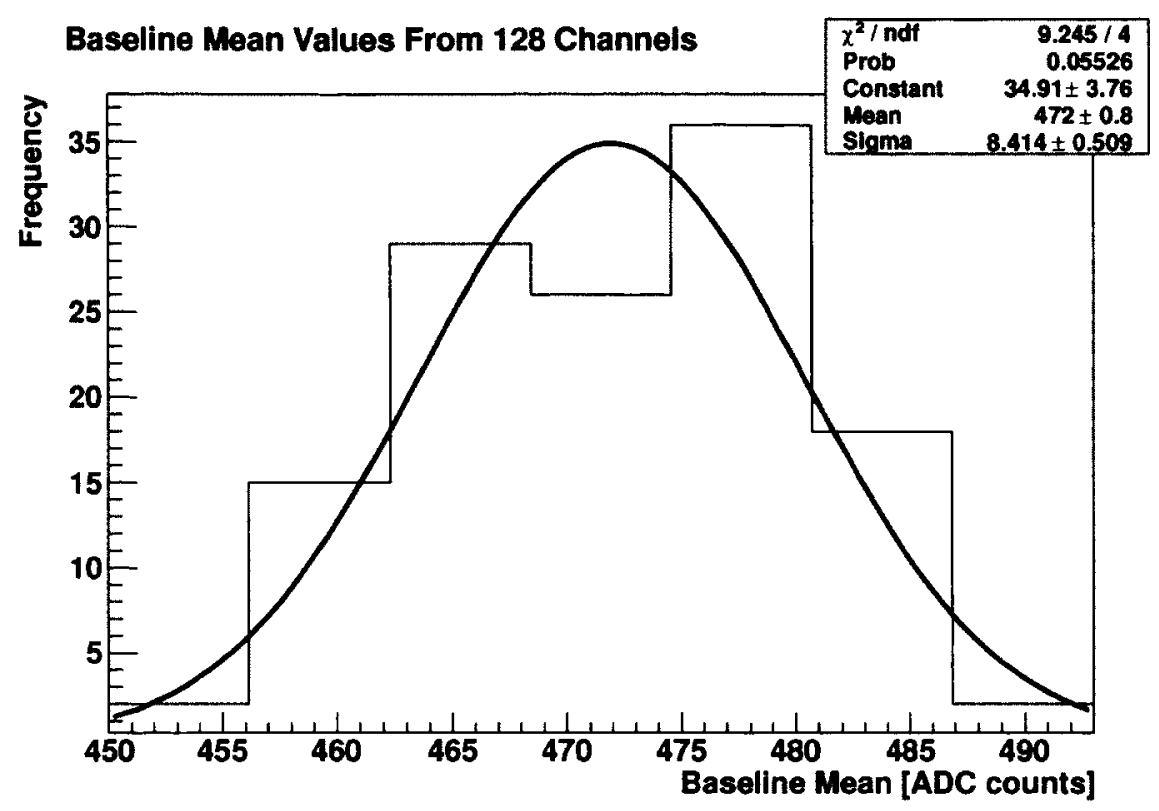

Figure 5.12: Distribution of the measured baselines for all 128 channels.

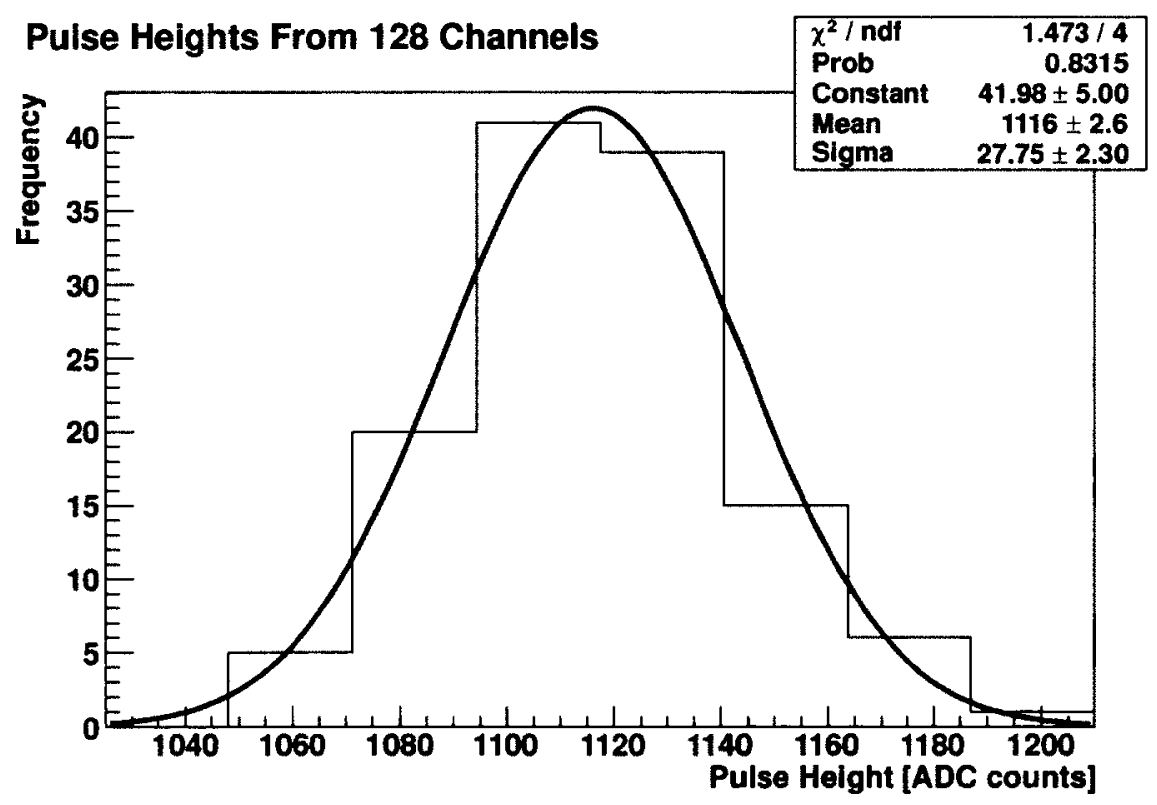

Figure 5.13: Distribution of the pulse heights for all 128 channels. 


\subsection{Vacuum evaporation chamber}

A critical piece of equipment involved in the construction of the detector package is our vacuum evaporation chamber. The chamber, shown in Figure 5.14 was constructed to perform evaporations of CsI powder onto the photocathode. It is a cylindrical stainless steel chamber with a $73.7 \mathrm{~cm}$ inside diameter and is open at the top. The chamber is $35 \mathrm{~cm}$ tall with three posts to hold the PCB and frame $30 \mathrm{~cm}$ above the base.

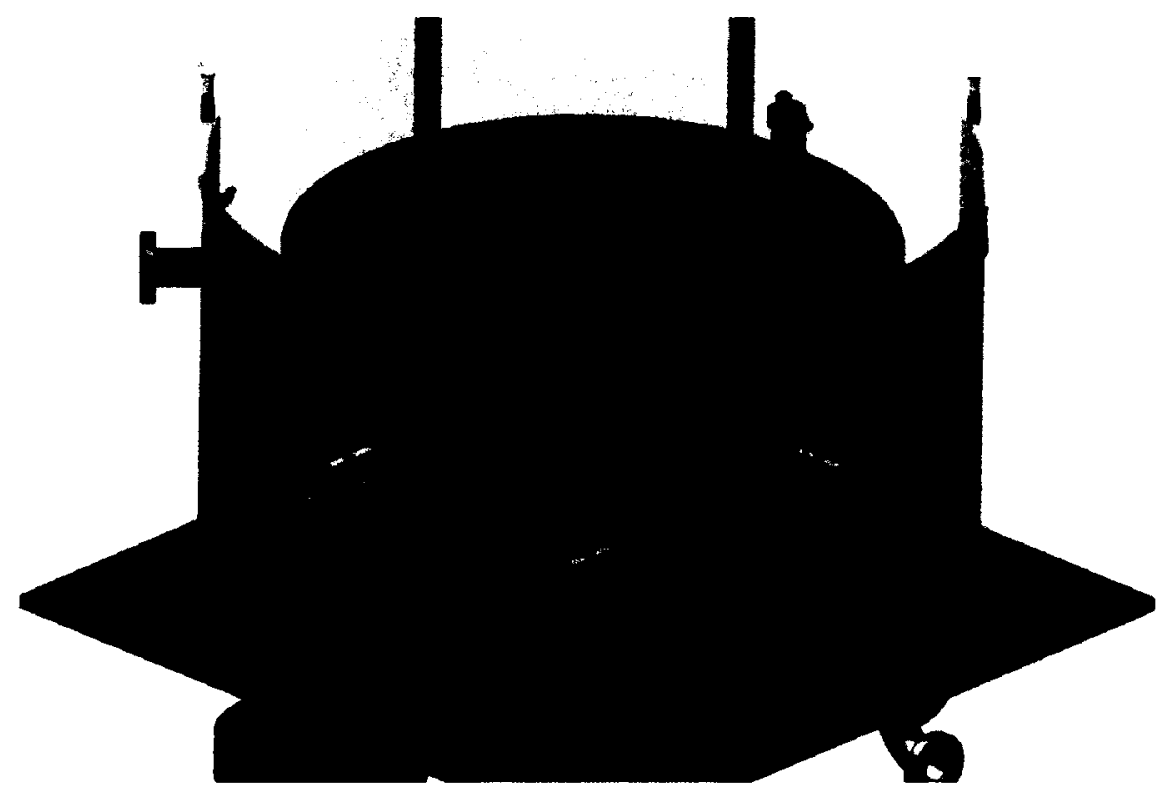

Figure 5.14: Cut-away of the vacuum evaporation chamber. Three of the molybdenum boats are visible; the other two are located in front of the two viewing ports, which can be seen in the lower portion of the chamber. The shutter system is also visible, shown here in the open position. The CsI readout board and frame are shown (brown) sitting near the top of the chamber.

The chamber can contain up to 5 molybdenum boats, electrically connected in series. Each boat is a thin rectangular piece of molybdenum which has a dimple punched in the centre; this dimple holds the CsI as it is heated. The four outer boats are evenly spaced around the inside wall of the chamber, and the fifth boat sits in the centre of the chamber. Each boat is covered by a shutter, and all of the shutters 
are connected and can be opened or closed as one. The boats are heated using a Magna-Power Electric power supply which can supply a $120 \mathrm{~A}$ output at $20 \mathrm{~V}$.

\subsubsection{Uniformity of CsI evaporations}

A series of measurements were made with the vacuum evaporation chamber to determine the dispersion pattern of CsI. All 5 boats in the chamber were used with the 4 outer boats containing roughly $150 \mathrm{mg}$ of CsI powder and the central boat containing roughly $30 \mathrm{mg}$. A model of the evaporation pattern was calculated using these experimental parameters (this model was developed by XEP member Francois Leonard, and modified by Dr. Chris Ouellet). This model was made by distributing the CsI mass isotropically over a solid angle of $2 \pi$, and then calculating the thickness on a $28 \mathrm{~cm}$ diameter horizontal surface sitting $30 \mathrm{~cm}$ above the CsI source. This model is shown in Figure 5.15. To test the model, the thickness was measured at several positions within the chamber. The measurements were made using an Inficon Q-pod Quartz Crystal Monitor. The results are summarized in Table 5.2. These data are plotted along side a scaled version of the model data Figure 5.16. The model data is scaled here so that the thickness is similar to the measurements; the important features to compare are the uniformities.

The same procedure was followed for each measurement with respect to the rate at which the boats were heated. During the measurements an erroneous reading of the deposition rate from the thickness monitor was noticed; the monitor often read between $-0.002 \mathrm{~nm} / \mathrm{s}$ and $0.002 \mathrm{~nm} / \mathrm{s}$, even when no material was being deposited. The error on the thickness reading is then estimated as the length of the run times $0.002 \mathrm{~nm} / \mathrm{s}$. Each run took roughly 45 minutes, so $(45 \mathrm{~min})(60 \mathrm{~s} / \mathrm{min})(0.002 \mathrm{~nm} / \mathrm{s})$ $=5.4 \mathrm{~nm}$ is the estimated error on the thickness. This rate was not constant, though, so this is likely an overestimate of the error on the readings. The position of the sensor within the chamber was measured to a precision of approximately $3 \mathrm{~mm}$. 


\section{Csil layer made by evaporation of 5 boats, $28 \mathrm{~cm}$ radius, $30 \mathrm{~cm}$ height}

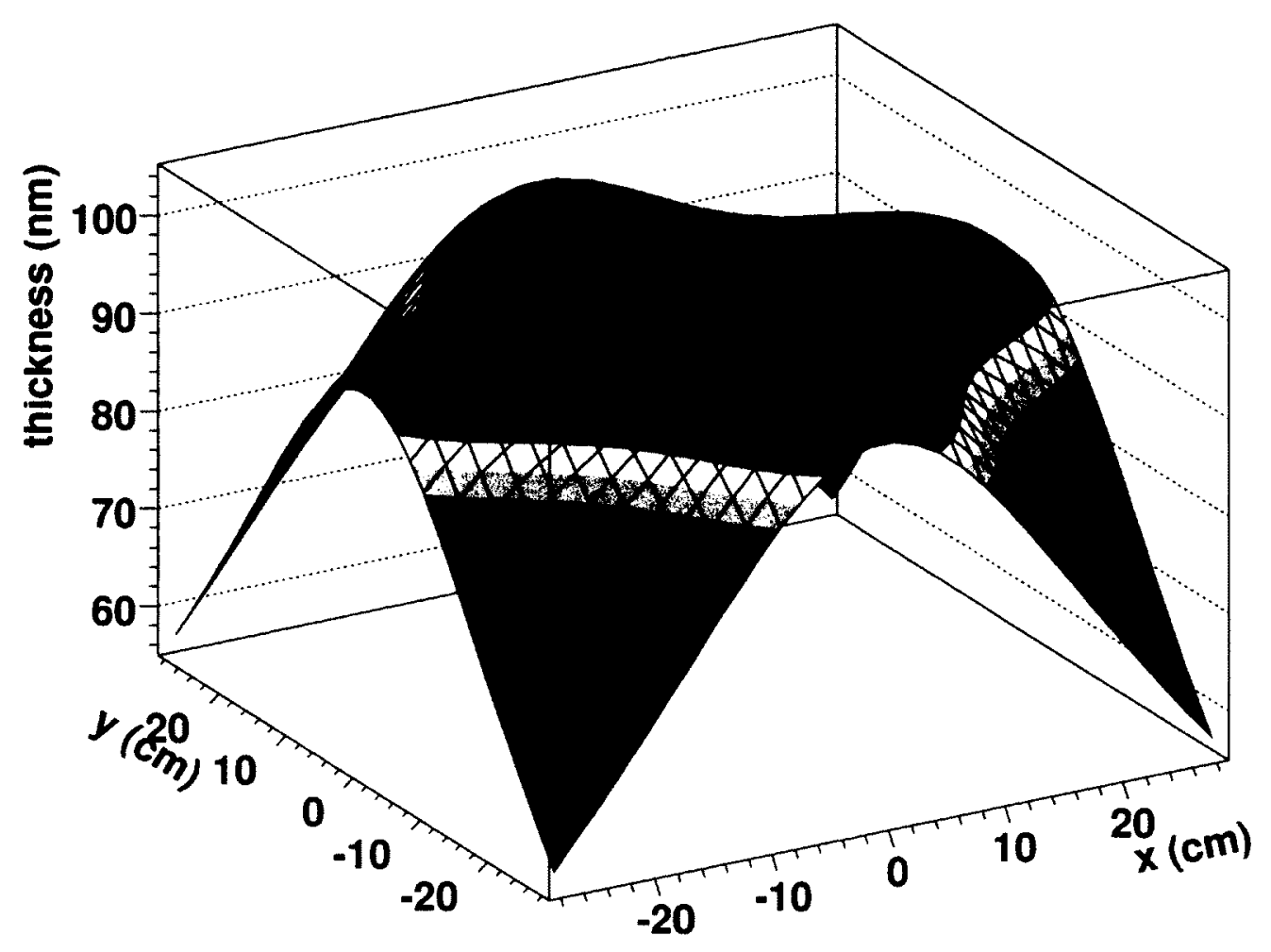

Figure 5.15: CsI dispersion pattern calculated using the 5-boat configuration of the vacuum evaporation chamber. The outer boats contain $150 \mathrm{mg}$ of CsI and the central boat contains $30 \mathrm{mg}$. The point $(0,0)$ represents the location of the central boat and the points $(0, \pm 28)$ and $( \pm 28,0)$ represent the locations of the four outer boats. The photocathode has a radius of $24.4 \mathrm{~cm}$; this is the portion of the deposition pattern we are interested in.

Another set of measurements was performed using just the central boat containing $150 \mathrm{mg}$ of CsI. A model of the pattern is shown in Figure 5.18. The results of these measurements can be found in Table 5.3, and these data are plotted in Figure 5.17. As with the 5-boat test, the model data is scaled to compare the uniformities more closely. These runs took much less time to complete than with the 5-boat configuration, taking about 15 minutes. The thickness error is estimated the same as before, $(15 \mathrm{~min})(60$ 


\begin{tabular}{cc}
\hline Position $[\mathrm{cm}]$ & Thickness $[\mathrm{nm}]$ \\
\hline \hline $29.0 \pm 0.3$ & $120.5 \pm 5.4$ \\
$27.0 \pm 0.3$ & $120.9 \pm 5.4$ \\
$22.5 \pm 0.3$ & $130.9 \pm 5.4$ \\
$18.0 \pm 0.3$ & $128.1 \pm 5.4$ \\
$13.5 \pm 0.3$ & $126.6 \pm 5.4$ \\
$9.0 \pm 0.3$ & $127.4 \pm 5.4$ \\
$4.5 \pm 0.3$ & $125.0 \pm 5.4$ \\
$0.0 \pm 0.3$ & $120.2 \pm 5.4$ \\
\hline
\end{tabular}

Table 5.2: Measured thickness of CsI deposition using the 5-boat configuration of the evaporation chamber.

$\mathrm{s} / \min )(0.002 \mathrm{~nm} / \mathrm{s})=1.8 \mathrm{~nm}$

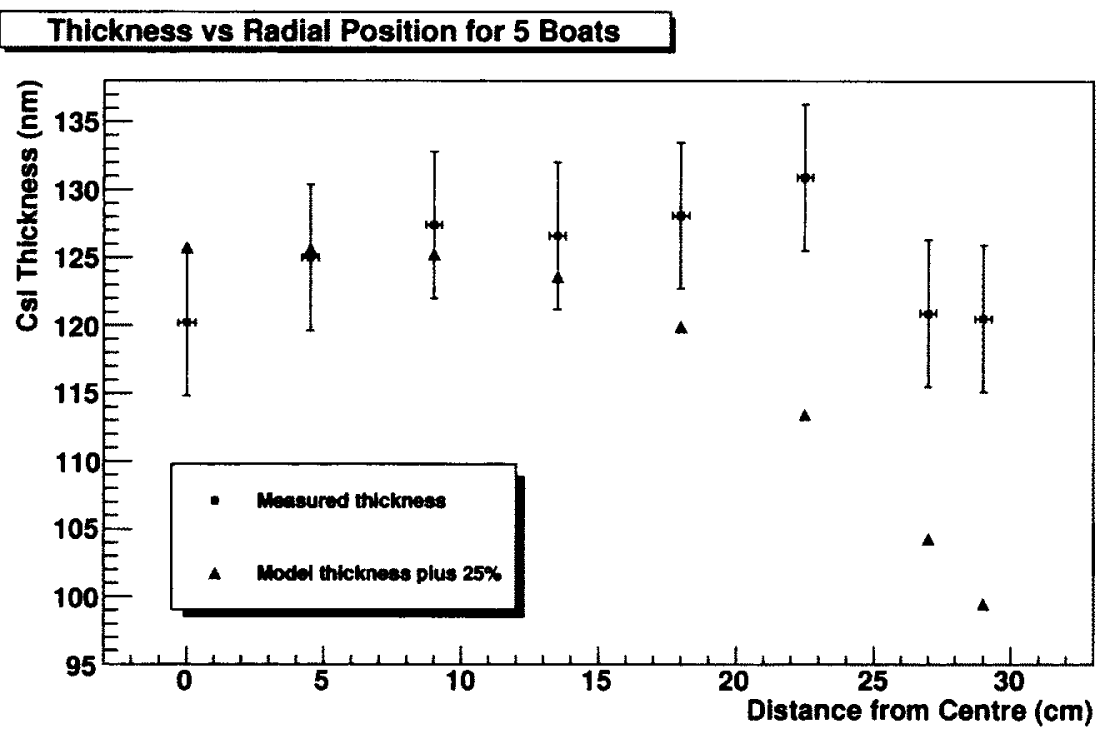

Figure 5.16: Measured 5-boat CsI dispersion pattern corresponding to Table 5.2. The model shown in Figure 5.15 is shown as well, though the values have been shifted by $25 \%$ to compare with the measured data. For reference, XEP's photocathode has a radius of $24.4 \mathrm{~cm}$. 


\section{Thickness vs Radial Position for 1 Boat}

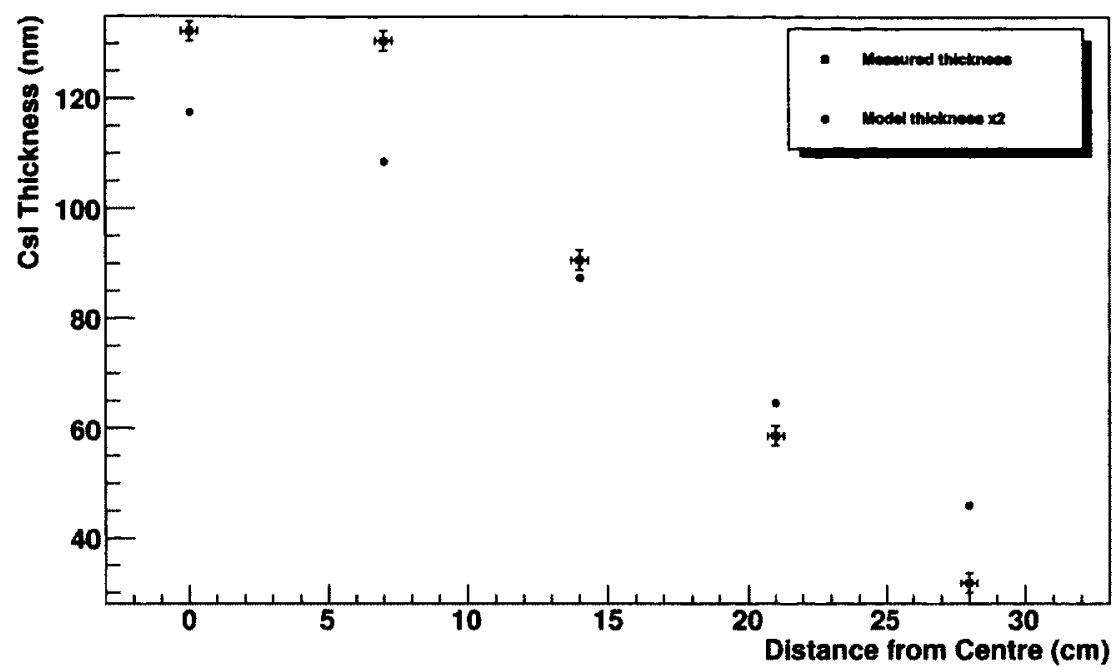

Figure 5.17: Measured 1-boat CsI dispersion pattern corresponding to Table 5.3. The model shown in Figure 5.18 is shown as well, though the values have been shifted by a factor of 2 to compare with the measured data.

\begin{tabular}{cc}
\hline Position [cm] & Thickness [nm] \\
\hline \hline $28.0 \pm 0.3$ & $31.8 \pm 1.8$ \\
$21.0 \pm 0.3$ & $58.6 \pm 1.8$ \\
$14.0 \pm 0.3$ & $90.6 \pm 1.8$ \\
$7.0 \pm 0.3$ & $130.6 \pm 1.8$ \\
$0.0 \pm 0.3$ & $132.3 \pm 1.8$ \\
\hline
\end{tabular}

Table 5.3: Measured thickness of CsI using only the central boat. 


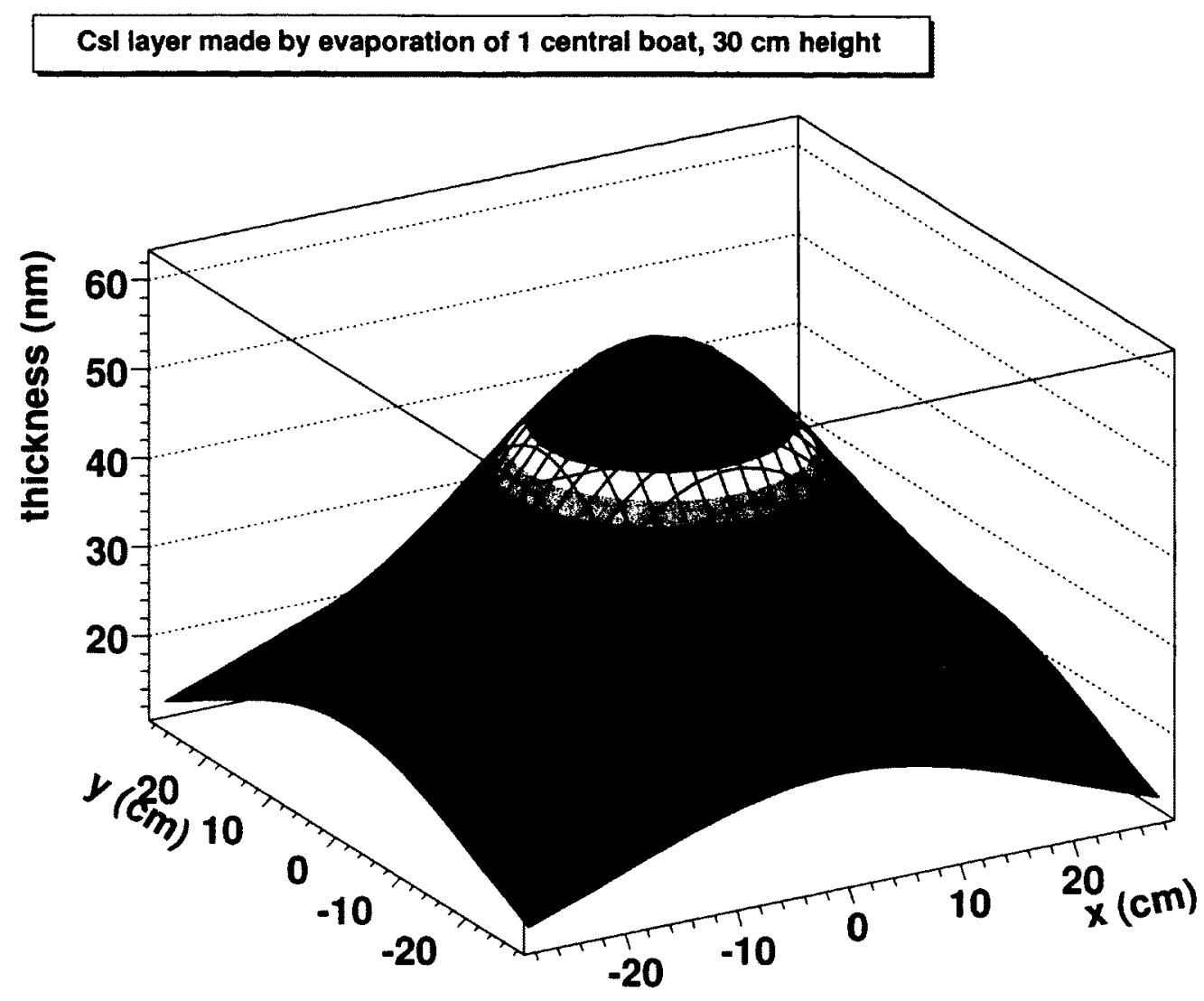

Figure 5.18: CsI dispersion pattern calculated using only the central boat containing $150 \mathrm{mg}$. The point $(0,0)$ represents the location of the boat.

From the measurements made using 5 -boats we see that there is a relative variation in the thickness of less than $10 \%$, ranging from $120.2 \mathrm{~nm}$ to $130.9 \mathrm{~nm}$. This variation is also likely a maximum that can be expected, as the measurements were taken along a line between two boats. Figure 5.15 shows the areas with the largest variation are those between any two adjacent outer boats. This uniformity is important because there is a relationship between the quantum efficiency of a CsI photocathode and the thickness of its CsI layer. It is very important that the CsI thickness on a pad be uniform so that the response is the same across the pad. It is also important that the pad-to-pad variations are minimal so that the response from all pads is the same. 
From Cusanno et. al [45] there appears to be a difference in quantum efficiency of $1 \%$ over the $120-130 \mathrm{~nm}$ thickness range.

The measurements made using a single boat show a sharper drop in thickness versus radius than the model. This is likely because the model calculates distribution of the CsI over a solid angle of $2 \pi$, when in reality the CsI sees less than this as it sits recessed inside the molybdenum boats. The first photocathode was produced with only the central boat containing Csl. From the centre of the photocathode to one of the far corners of the $4 \times 4$ pad grid is $7.3 \mathrm{~cm}$. Over this distance, the measurements show a difference in thickness of a few $\mathrm{nm}$. This means that the first XEP photocathode should have negligible differences in the quantum efficiency between pads as a result of thickness variations.

\subsection{Creating a conductive grid on fused silica using photolithography}

The possibility of using photolithography to apply a series of conductive aluminum lines on fused silica was investigated. These aluminum lines would act as the ground potential side of the electroluminescence region and replace the grounded stainless steel wire grid shown in Figure 4.3. Photolithography is a multi-stage process which is used to produce a chemical mask, typically on circuit boards. Such a circuit board would then undergo metallization to produce the desired circuit while the mask protects the areas of the substrate where metal was not wanted. To start, a UVsensitive chemical photoresist is applied to the substrate and baked on. A UV source is then used to expose the desired circuit pattern into the photoresist; this chemically alters the photoresist making it soluble to a chemical developer. After development, any photoresist that underwent UV exposure is removed and metallization may occur. 
After metallization, the remaining photoresist is dissolved away, taking the unwanted metal with it.

Tests were performed on two different pieces of fused silica. The first was a sample puck from Corning about $2.5 \mathrm{~cm}$ in diameter; the second was a $10 \mathrm{~cm}$ diameter piece mounted in a stainless steel frame. To expose the desired pattern into the photoresist once it was applied, a Mylar mask was used with the negative image of the pattern printed on it. The mask had gaps of $0.25 \mathrm{~mm}$ at a pitch of $2.5 \mathrm{~mm}$ to match the desired line width and spacing. A $100 \mathrm{~W} \mathrm{Hg}$ vapour lamp was used for UV exposure inside a light-tight box. Too little UV exposure will not penetrate the photoresist down to the substrate, and too much will expose photoresist under the mask. Various exposure times were tested, as well as the spacing between lamp and substrate, to find the optimal balance. Aluminum was applied using the vacuum evaporator containing a tungsten coil. Rods of $99.999 \%$ pure aluminum were placed in the coil, melted, and evaporated onto the substrate. A series of photographs of 3 successful tests are shown in Figure 5.19. Hydrochloric acid was used to remove the aluminum from the fused silica between tests. It was fairly straightforward to fully remove a pattern, thus at full scale it would be simple to reset and test different grid geometries on a window.

This technique looks promising as a means of applying fine metal lines on fused silica. Though this initial testing involved a lot of trial and error to determine the optimal photoresist coating technique and UV exposure times, the results are encouraging. It is clear that very fine lines can be produced, and that aluminum will adhere to fused silica and form a conductive layer. It is also clear that specialized equipment would be required to produce lines at the $\sim 50 \mathrm{~cm}$ scale of XEP's windows. The potential exists to have the piece manufactured commercially, though since the typical use of the technique is for the production of circuit boards (up to $10 \mathrm{~cm}$ diameter), most companies are not equipped to deal with a piece as large as our window. Many companies throughout North America were researched, and there are at least 
two who perform the type of large-scale photolithography we require. The cost for commercial production is very high, though, and we want the ability to test different grid geometries. For the interim, it was decided that the ground side of the EL region would consist of a second wire grid assembly mounted in front of the window while this technique can be investigated further.

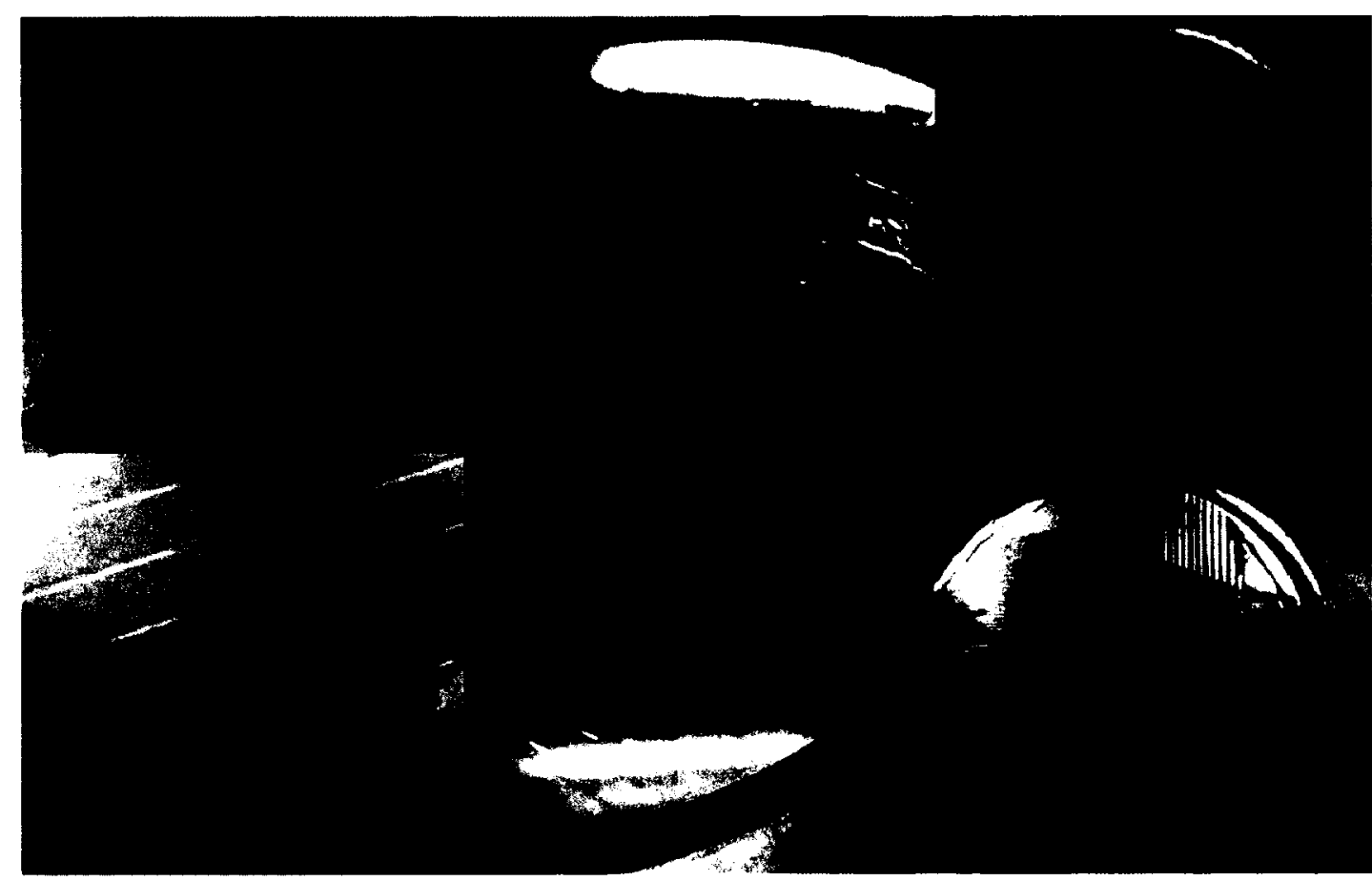

Figure 5.19: Photographs of 3 successful photolithography tests. Top left: The fused silica sample puck with photoresist mask, and after being coated with aluminum. The mask used was a scrap from the mask used with the larger test piece and was not large enough to cover the whole puck; the lines are 0.25 $\mathrm{mm}$ wide. Top centre: The excess aluminum being removed as the photoresist underneath it is dissolved. Top right: All of the photoresist has been removed, leaving just the desired aluminium pattern. Bottom left: The larger fused silica sample shown with photoresist and aluminum. Bottom centre: After removal of photoresist and excess aluminum. Though the lines are not fully intact, they are conductive. Bottom right: A different pattern with very fine structures that was attempted on the sample puck, shown after being coated in aluminium. 


\section{Chapter 6}

\section{Signals From the First XEP Detector Package - Results and Analysis}

The measurements from the first CsI photocathode were performed using a PerkinElmer PAX-1003-1 xenon flash lamp coupled to a fibre optic cable. The lamp has a maximum power output of $10 \mathrm{~W}$ and a stability of $\sim 1 \%$ at maximum intensity. It produces an output spectrum from $120 \mathrm{~nm}$ to $>2000 \mathrm{~nm}$. The other end of the fibre was held in place just under the fused silica window with a separation of $\sim 1$ $\mathrm{mm}$ (see Figures 6.1 and 6.2) to direct the UV output of the lamp to the pads. As stated in Chapter 4, for ease of comparison to the primary literature regarding CsI photocathodes, the $\mathrm{CH}_{4}$ pressures are stated in units of Torr, rather than mbar. Data was taken with $\mathrm{CH}_{4}$ in the detector package at pressures of 20 and 100 Torr for those pads which produced signals which were large enough to separate from the noise when no gain was applied. Several different quantities were measured. Gain curves for the two pressures were measured, along with a measurement of the maximum possible gain. The effects of signal and pad degradation were also investigated. In addition, the response at several locations across a pad was measured. Figure 6.3 shows a schematic of the overall set-up.

The signals generated by the CsI photocathode were passed through an Ortec 
142B pre-amplifier. These signals contained significant noise which was traced, in part at least, to the flash lamp's power supply. An attempt was made to shield the power supply, but the noise reduction was minor. To better filter this noise, the signal was passed through an Ortec 572 amplifier. While this removed many of the large variations in the signal, there was still some noise present in the baseline. The amplifier was set to a coarse gain of $20 \mathrm{x}$ and a shaping time of $6 \mu \mathrm{s}$. The signals were then collected using a Tektronix DPO 5054 oscilloscope. The oscilloscope was set to sample at $100 \mathrm{MSample} / \mathrm{s}$ over a period of $50 \mu \mathrm{s}$, generating 5000 samples per pulse with a spacing of $10 \mathrm{~ns} / \mathrm{sample}$.

The reader must note that all quoted values of $\mathrm{E} / \mathrm{p}$ are in units of $\mathrm{V} / \mathrm{mm}$.Torr, though these units will be omitted throughout the text for brevity.

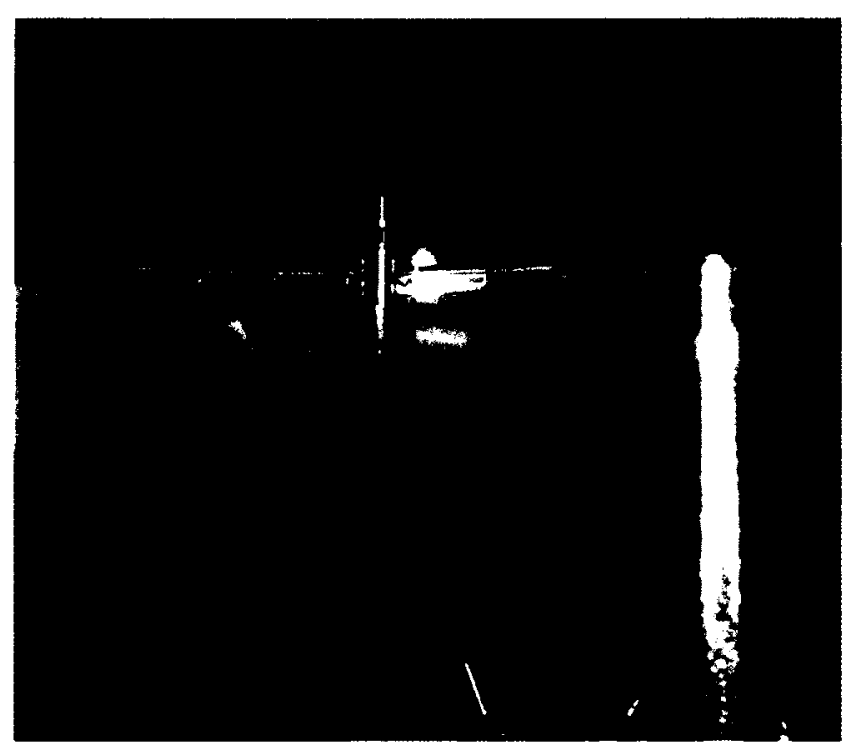

Figure 6.1: The set-up used to hold the fibre optic cable to the detector package. The cable is fed through a short length of pipe and held in place at the top with a clip. The pipe is held by an adjustable clamp on a retort stand so that the distance from the cable to the window can be adjusted. 


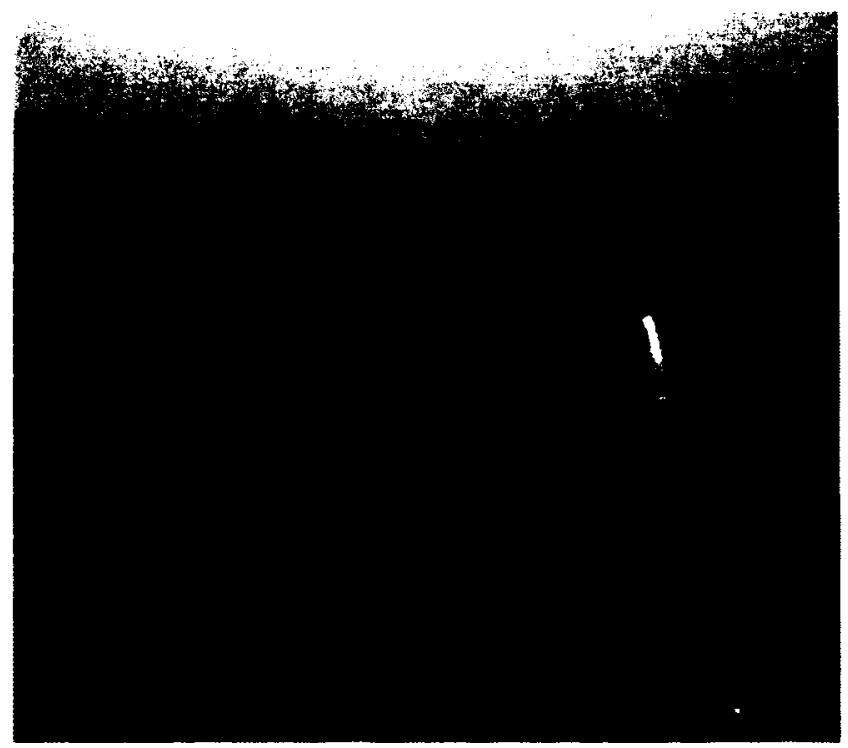

Figure 6.2: The fibre optic cable under the $4 \mathrm{x} 4$ grid in the Teflon spacer. The location of UV exposure of a pad can easily be adjusted by moving the retort stand.

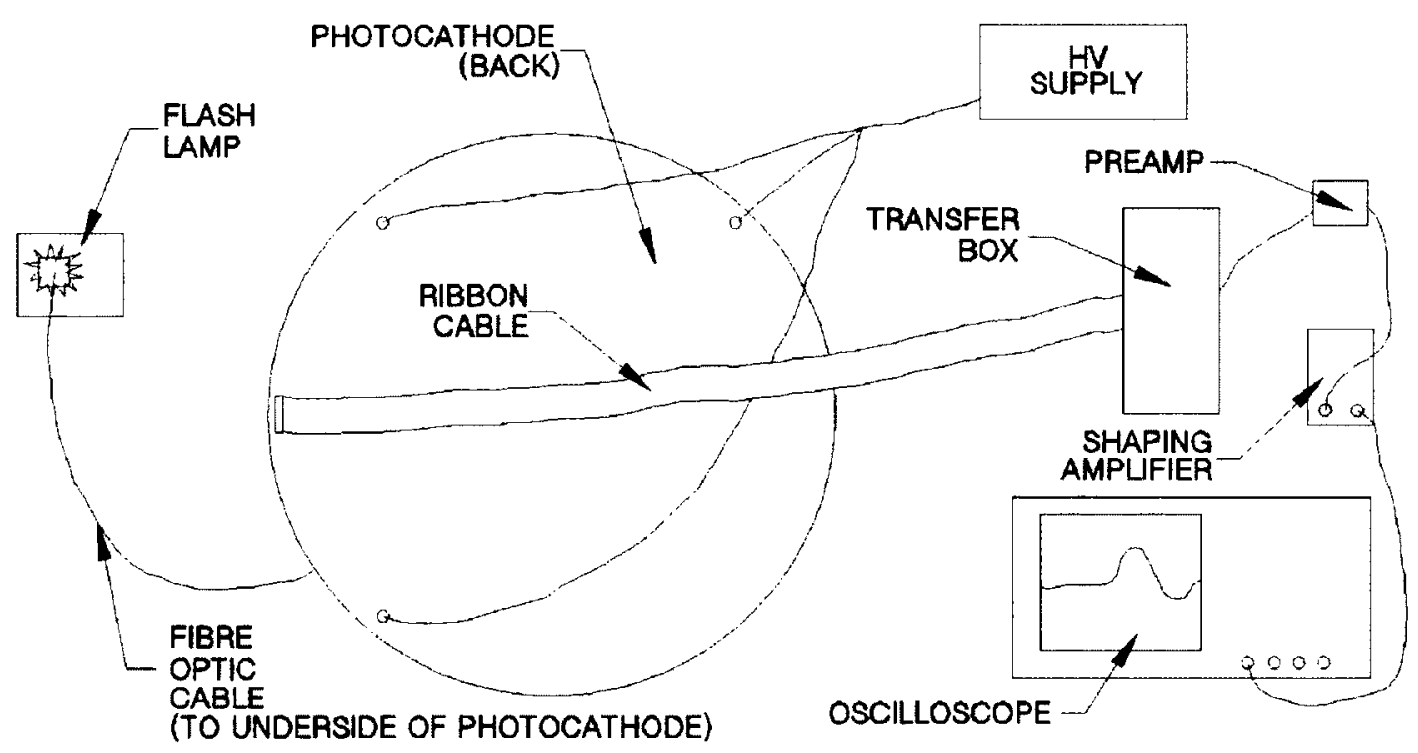

Figure 6.3: Schematic of the set-up used to characterize the first detector package. The CsI is on the opposite side of the photocathode of that shown. Not shown is the dark box that the photocathode was sitting on. 


\subsection{CsI response in methane}

The effect of operating the CsI photocathode in low-pressure $\mathrm{CH}_{4}$ with different reduced electric fields, E/p, was investigated. Two sets of tests were performed: the first set at $\mathrm{C} \mathrm{CH}_{4}$ pressure of 100 Torr, the second at 20 Torr. The pads B15, B22, and B31 were examined (see Figure 4.8 for the pad numbering). For data collection, the fibre optic cable was positioned roughly in the centre of each pad and the intensity of the flash lamp set to the maximum. 5000 pulses were recorded for each value of E/p. Each pulse is fit with a Gaussian to determine the height of the peak. Figure 6.4 shows a sample pulse with a Gaussian fit. The average of the pre-pulse baseline is then calculated from the first 2000 samples and subtracted from the Gaussian peak height to determine the height of the pulse.

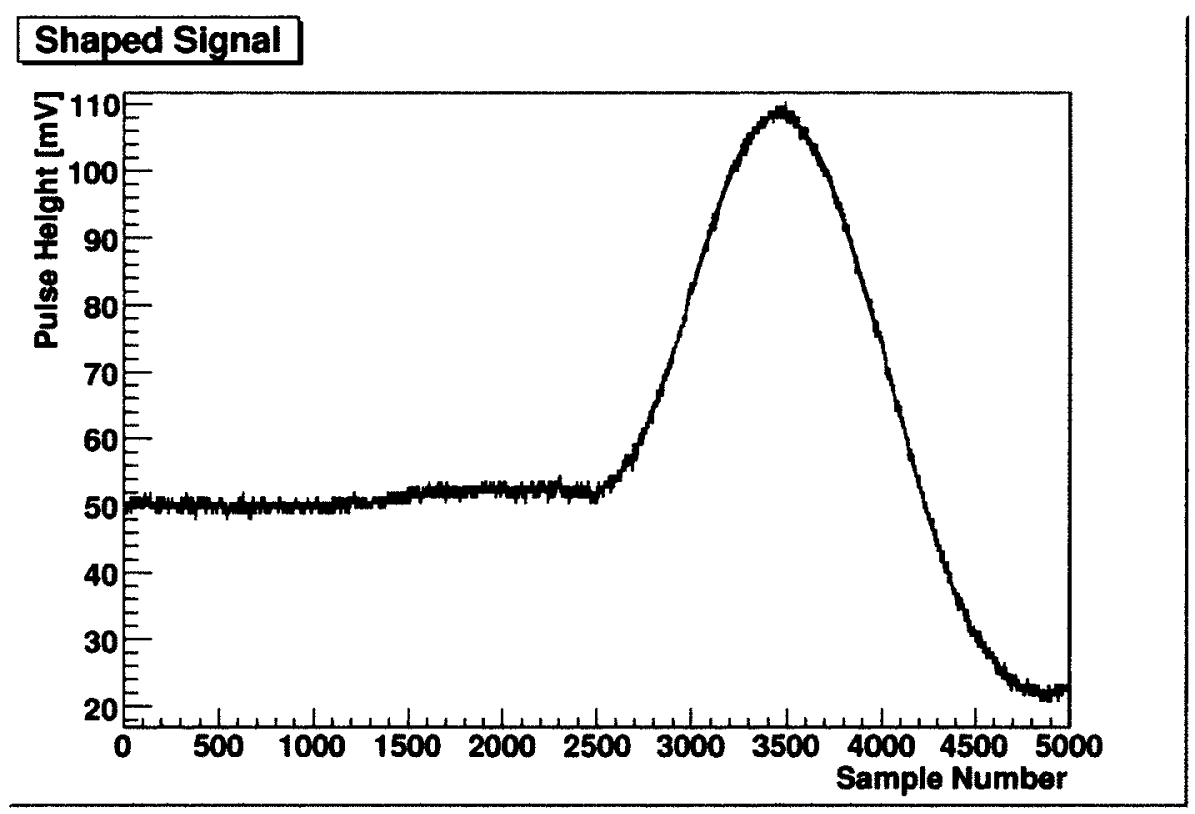

Figure 6.4: A sample pulse taken at a pressure of 100 Torr and a voltage of $635 \mathrm{~V}$. A Gaussian curve is fit to the peak to determine the peak height.

It was observed early on that the measured pulse height decreased over time. There are two main effects thought to be responsible for this. The first of these 
effects is degradation of the CsI due to both the absorption of water and the very high intensity of UV illuminating the pads. The second effect is thought to be gradual contamination of the methane by, for example, water and oxygen. These effects will be discussed in more detail later.

The signal drift was corrected in two steps. To correct for the drift during a run, the height of each pulse was plotted against its event number to get a distribution in time. A second-order polynomial was fit to this distribution, and the fit was used to adjust each pulse height. The adjustment was such that a second fit to a plot of the distribution in time was consistent with a flat line, and having a $y$-intercept equal to that of the first fit.

To correct for the drift between runs, a small data set of 500 events was taken before each run at the lowest E/p value (0.8 at 100 Torr, corresponding to an applied voltage on the mesh of $254 \mathrm{~V}$, and 1.0 at 20 Torr, corresponding to a voltage of 64 $\mathrm{V})$. The mean of a Gaussian fit to each distribution of pulse heights was compared to the first data set taken and a correction factor determined from the ratio of pulse heights for each $\mathrm{E} / \mathrm{p}$ value. An example plot showing the original data and fits along with this series of corrections is shown in Figure 6.5 for the 100 Torr $1111 \mathrm{~V}$ data set. 

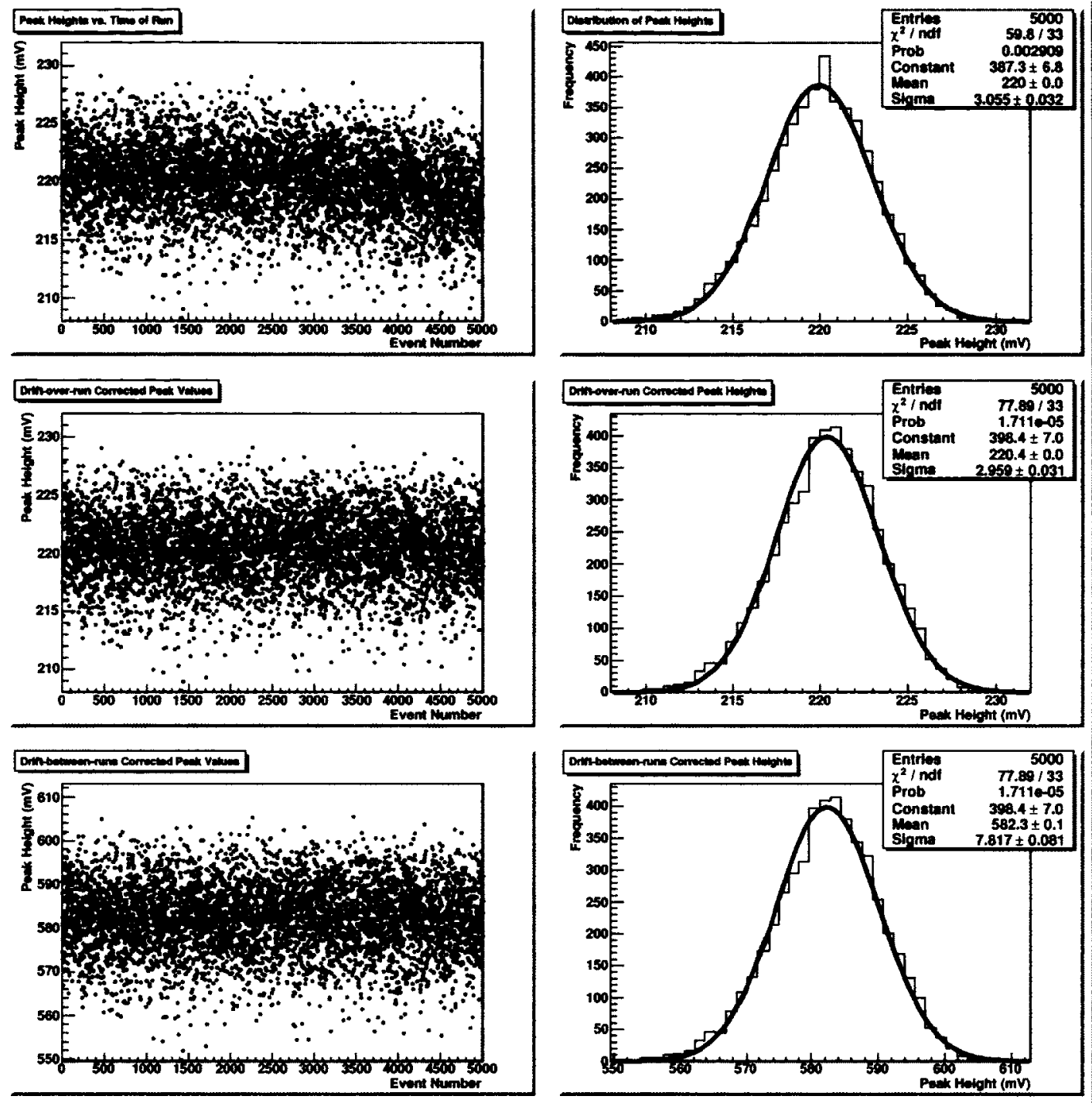

Figure 6.5: Successive pulse height corrections from pad B22 at $1111 \mathrm{~V}$ in 100 Torr. The top two plots show the unaltered pulse height distributions. It is clear that the pulse heights trend downward over time, so a second-order polynomial is fit to the distribution in time. This drift is corrected in the two middle plots by adjusting the pulse heights so that a second-order polynomial fit returns a flat line. The final correction, shown in the bottom two plots, is made by multiplying all the shifted pulse heights by a correction factor. 


\subsubsection{Torr gain curves}

Figure 6.6 shows the measured peak heights for each 500 event pre-run data set used to correct for the signal decrease between runs. The points are spaced evenly in the figure, though this is not truly the case. The time between runs was roughly 30 minutes but was not consistent. The values for Run 1 are the initial pulse heights to which subsequent values are corrected. These runs were taken before the first 5000 event runs at $254 \mathrm{~V}$ for each pad. The values for Run 13 were taken after the 1111 V run.

After the final runs the detector package was evacuated and pumped down over about 12 hours. It was the refilled with $\mathrm{CH}_{4}$ and another $254 \mathrm{~V}$ run was taken. The fibre was not moved until all data was collected, so the same area of each pad was illuminated for each run. These runs are shown as Run 14 in Figure 6.6. Each of these runs in new $\mathrm{CH}_{4}$ shows an increased pulse height over the post-1111 $\mathrm{V}$ runs of $\sim 20-30 \%$. This may indicate that some of the total signal degradation was due to contamination of the $\mathrm{CH}_{4}$, but also that there may be other effects at work. Figure 6.7 shows the correction factors for each pad.

Since this signal degradation occurs over time it may be due to contaminants such as water and oxygen in the detector package which were not present in the $\mathrm{CH}_{4}$ bottle. It is possible that some of the interior detector package components are outgassing into the methane volume. Both the o-ring used to seal the window and the Teflon spacer may be outgassing into the detector volume. These contaminants could affect the signal in two ways: adsorption to the CsI surface (by water) and collection of drifting electrons (by oxygen). The most significant of these two effects is likely the degradation of the CsI surface. The recovery of the pulse heights seen in Figure 6.6 suggests that some of these contaminants may be removed from the surface of the CsI. It is clear, however, that there is some permanent damage to the CsI. Another 
effect that may be responsible for is damage to the CsI caused by the high intensity, broad spectrum EM radiation produced by the lamp.

This contamination of the $\mathrm{CH}_{4}$ should not be an issue in the detector package's ultimate configuration for a couple of reasons. The first reason is that the o-ring will not be present as the window will be sealed to the detector package frame using an epoxy rated for use in high-vacuum. The second reason is that a system is planned which will flow and purify the methane, and this should remove any contaminants that the Teflon spacer may introduce.

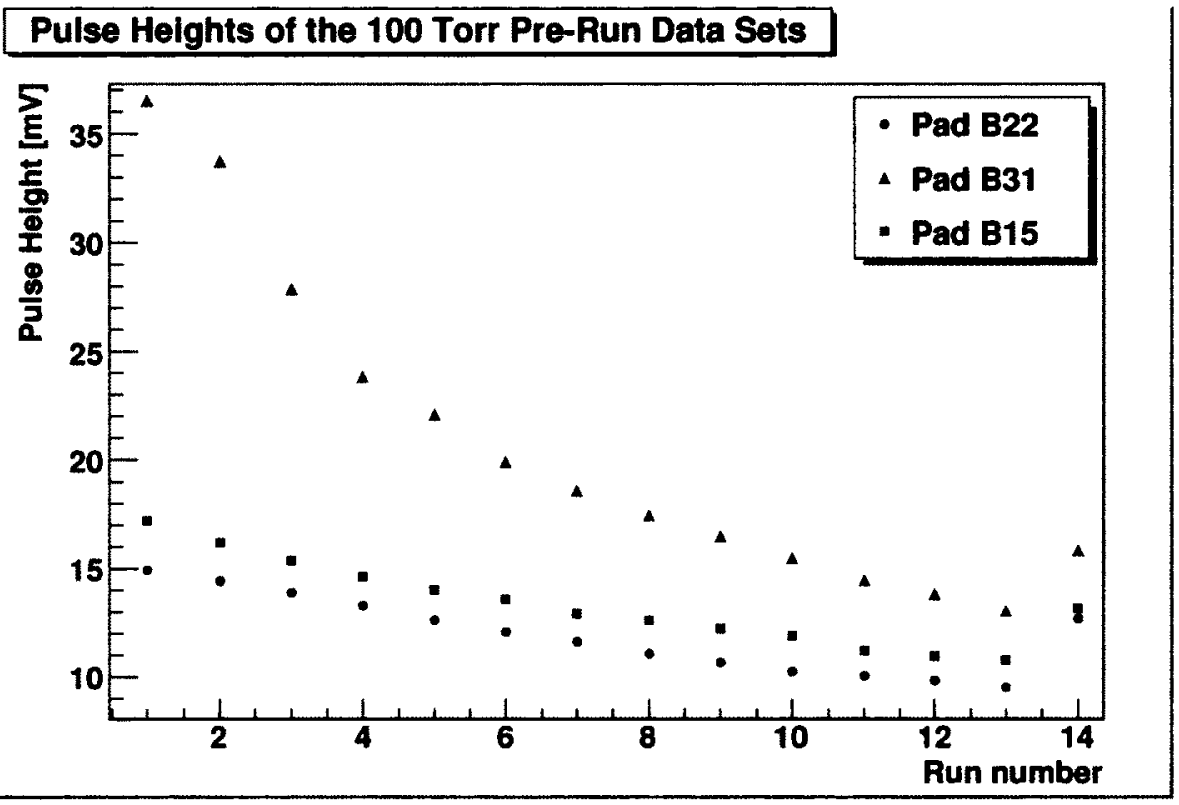

Figure 6.6: Peak heights from the 100 Torr pre-run data sets. The time between points is not constant, though for all but the last point the spacing is roughly 30 minutes. The points for Run 1 for each data set is used as the initial pulse height to correct subsequent runs. The points for Run 13 were taken after the final runs at $1111 \mathrm{~V}$. The points for Run 14 were taken the following day after the detector package had been evacuated and re-filled with clean $\mathrm{CH}_{4}$. 


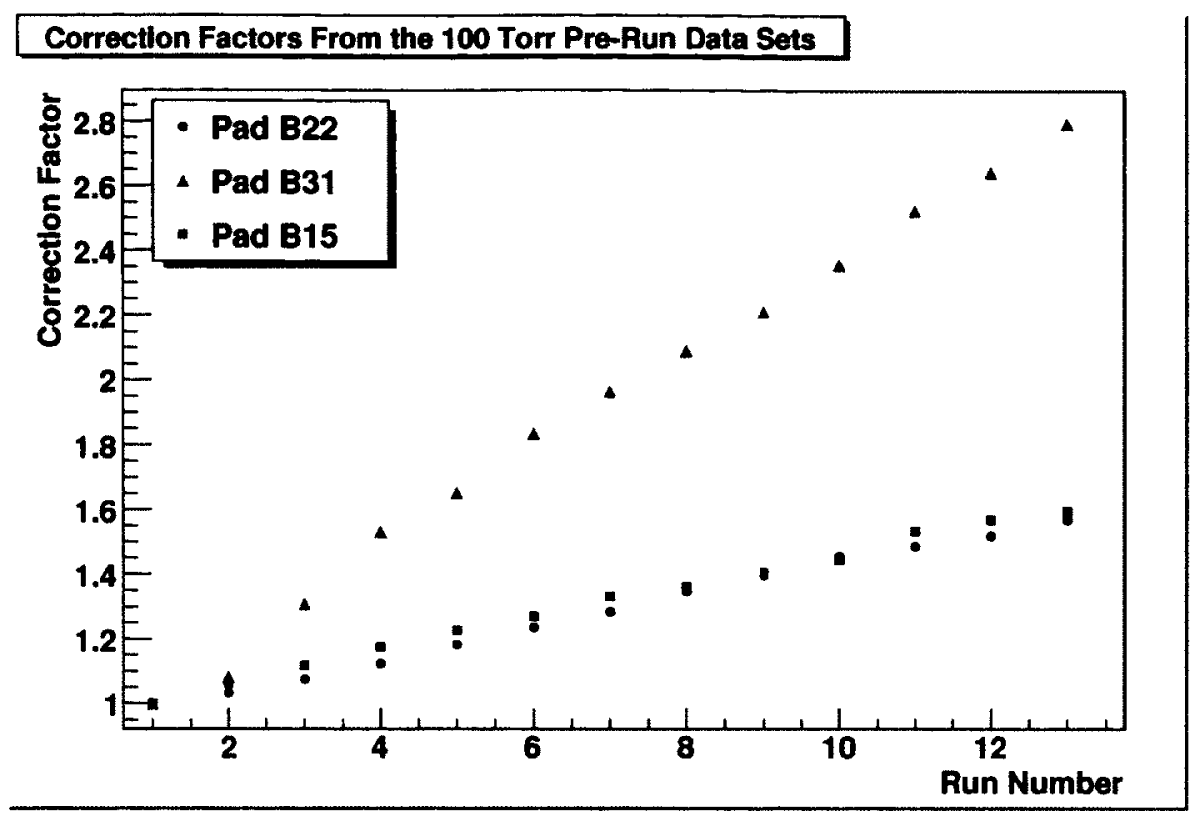

Figure 6.7: Corrections factors from the 100 Torr pre-run data sets. The time between runs is roughly 30 minutes, but is not consistent.

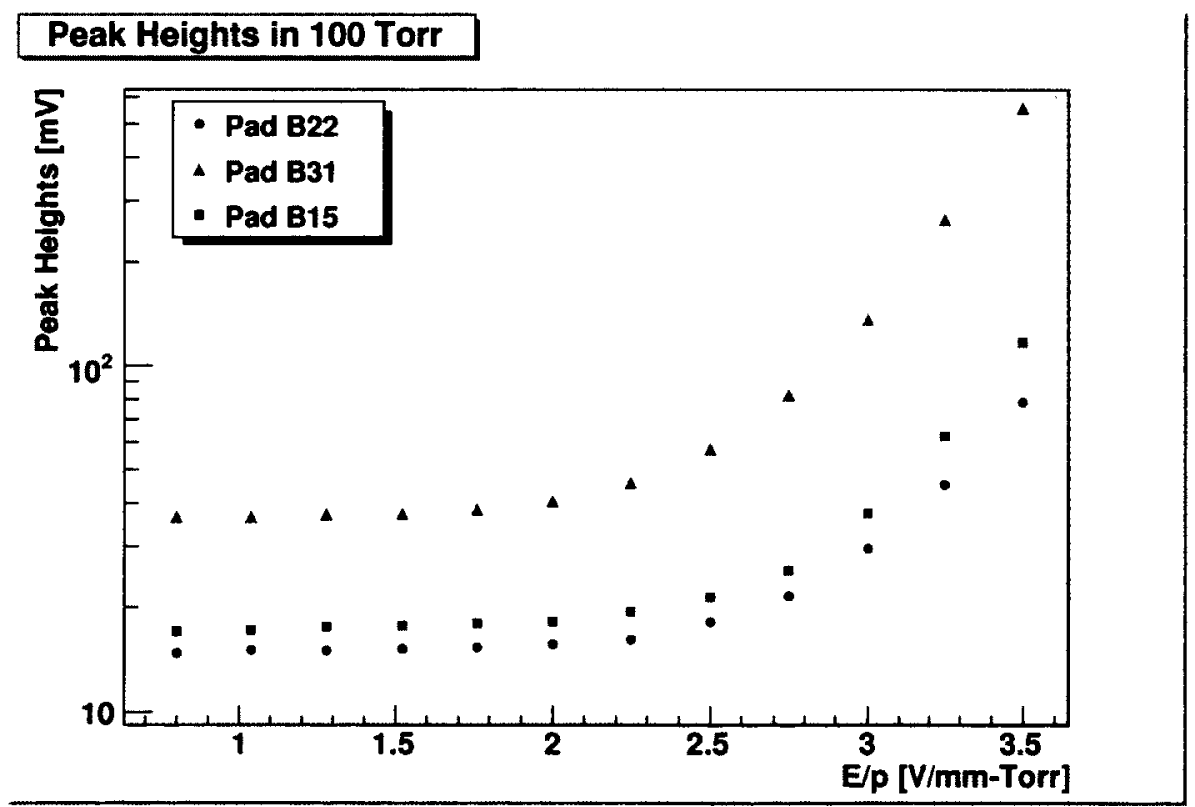

Figure 6.8: Pulse height as a function of $\mathrm{E} / \mathrm{p}$ for 3 pads in 100 Torr. The fit errors are too small to be seen at this scale. 
As seen in Figure 6.8 the magnitude of the pulses from each pad differs, though the general response with respect to $E / p$ is similar between the three pads. Figure 6.9 shows the gain values for each pad calculated from the ratio of the pulse height at the lowest $\mathrm{E} / \mathrm{p}$ value to each subsequent pulse height. Figure 6.10 shows the same data focused on the no-gain region.

In the no gain region, after the pulse heights have been corrected, there is a slight upward trend in the pulse heights. This trend is also seen by Anderson [42]. The trend is expected as it was noted in Section 3.3 that the quantum efficiency of a CsI photocathode can increase with increasing electric field. A more precise theory is that with increasing electric field there is a greater efficiency of removing photoelectrons from the bulk of the CsI, rather than incoming photons producing more photoelectrons. In this case the effect would be an apparent increase in the quantum efficiency.

When the gain from each pad is examined, pad B31 appears to begin producing gain at a lower $\mathrm{E} / \mathrm{p}$ value than $\mathrm{B} 15$ and B22. This is most obvious in Figure 6.10 which is focused around the no-gain region. In Figure 4.8 it can be seen that there are ripples in the nickel mesh; this may account for the earlier onset of gain compared to Anderson as a lesser electric field gap than expected would cause the calculated $\mathrm{E} / \mathrm{p}$ values to be different than the real values. In comparison, the mesh beneath pads B15 and B22 appears to be flat. These ripples may account for the difference in the gain curves. This explanation has been considered in Figure 6.11 by using a reduced field gap to calculate the $\mathrm{E} / \mathrm{p}$ values for pad B31. The gap is reduced by 0.3 $\mathrm{mm}$ to a value of $2.875 \mathrm{~mm}$ in the calculation, while the other two pads maintain the original gap of $3.175 \mathrm{~mm}$. The gain curve for pad B31 can now be seen to be similar to those of the other two pads. It is clear that small changes in the field gap can have a significant effect on the gain, so it is critical for XEP's energy resolution goals that a uniform electric field is present. 


\section{Torr Gain Curves}

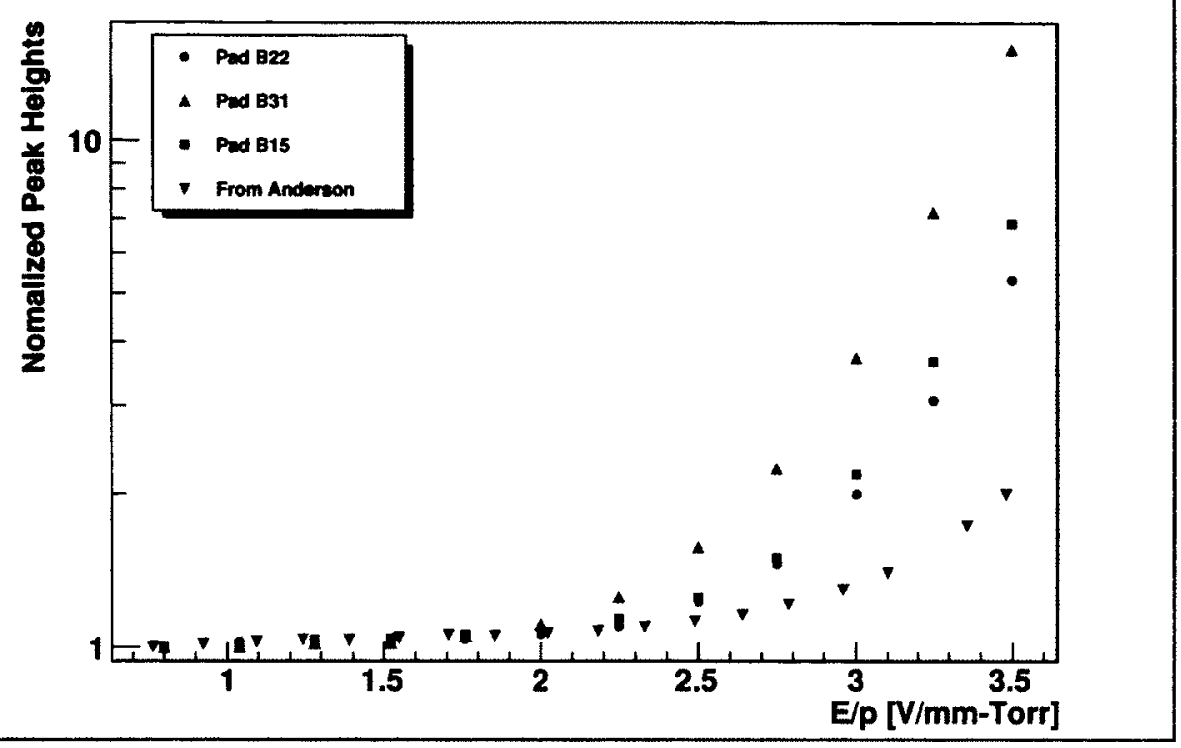

Figure 6.9: The same values from Figure 6.8 are shown, with the pulse heights for each pad given as a ratio to the value at $\mathrm{E} / \mathrm{p}=0.8$ to calculate gain. Also shown are values from Anderson [42] which have also been reduced to the value closest to $\mathrm{E} / \mathrm{p}=0.8$. The fit errors are too small to be seen at this scale.

While the difference in gain between $\mathrm{B} 31$ and B15,22 is large, the same is true for the difference between B15,22 and the Anderson data. Despite the differences in gain, the onset occurs around the same $E / p$ value for each data set. 


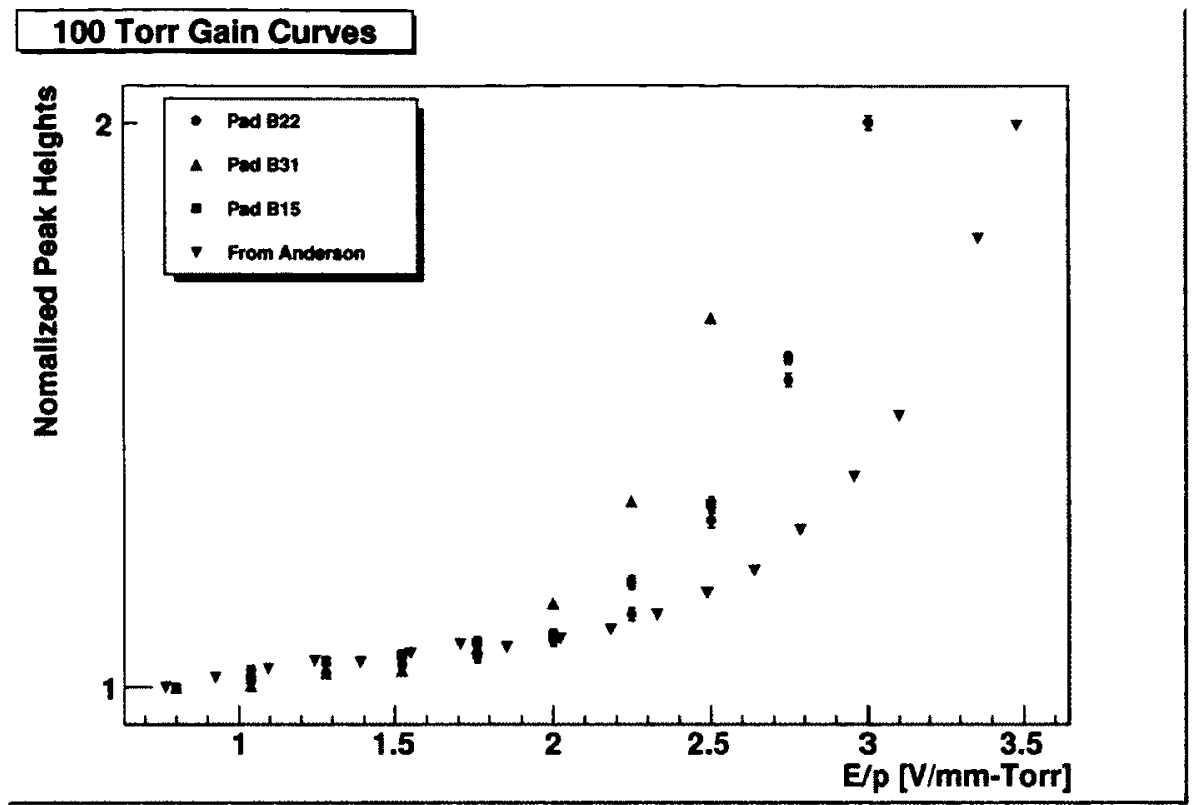

Figure 6.10: The same plot from Figure 6.9 focused to show the no-gain region and the Anderson data [42]. The fit errors for pad B31 are too small to be seen at this scale. 


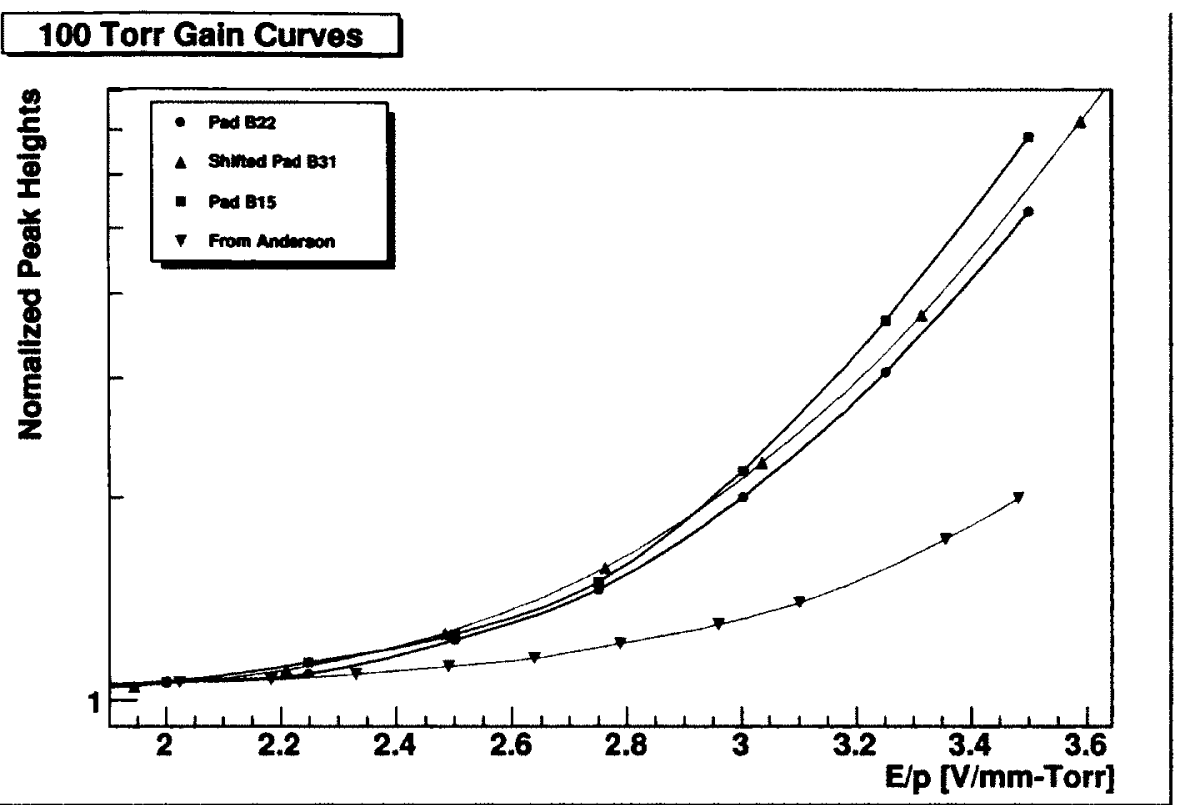

Figure 6.11: The effect of reducing the electric field gap for pad B31 while maintaining that for B15 and B22 is examined here. The gap for pad B31 has been reduced in the $\mathrm{E} / \mathrm{p}$ calculation by $0.3 \mathrm{~mm}$ to a value of $2.875 \mathrm{~mm}$ such that the gain curve follows that of the other two pads. The lines are not fits to the data, but are simply curves drawn to highlight the general trends of the data.

The energy resolution of each pad can be calculated from the pulse height distribution fits. These values are shown in Figure 6.12. Again, as with the pulse heights, the resolution between pads varies significantly, but the overall trends with respect to $\mathrm{E} / \mathrm{p}$ is the same for each pad. A deterioration of the energy resolution is seen for signals in the no gain region from the $\mathrm{E} / \mathrm{p}$ values of 0.8 to 2 . The reason for this is not understood at this time.

In comparison, an improvement of the energy resolution with increasing $\mathrm{E} / \mathrm{p}$ is seen when gas gain begins to occur, from the $\mathrm{E} / \mathrm{p}$ values of $>2$ to 3.5 . In the no-gain region, electronic noise is likely contributing substantially to the pulses. This noise is constant, however, so in the gain region the noise becomes a smaller fraction of the pulse heights. At high enough gain, the electronic noise becomes negligible and the signal is mainly limited by a combination of the stability of the flash lamp and of the 
resolution of the photocathode.

Looking back to the middle plot of Figure 6.5, the variation in the measured pulse heights does not appear to change significantly during the run after the drift is corrected for. It is therefore reasonable to say that the energy resolution does not change during a run.

\section{Pad Resolutions in 100 Torr}

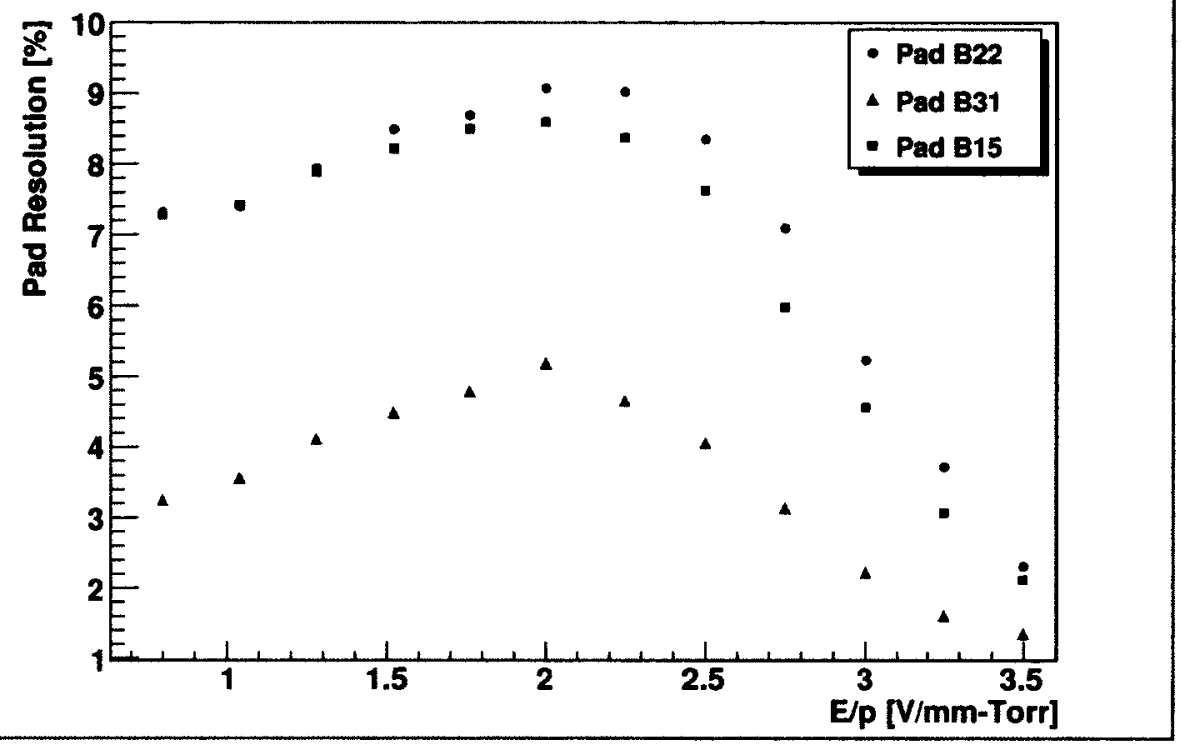

Figure 6.12: The calculated resolution of 3 CsI pads in 100 Torr as a function of $\mathrm{E} / \mathrm{p}$. The fit errors are too small to be seen at this scale.

\subsubsection{Torr gain curves}

After the 100 Torr measurements were completed, the same tests were repeated in $\mathrm{CH}_{4}$ at 20 Torr. At this time, unfortunately, channel B22 was no longer producing a large enough signal in the no-gain region to clearly distinguish from the baseline noise. It was decided that data would not be taken on this pad at 20 Torr. The first noticeable difference from the 100 Torr data measurements is that now pad B31 is producing a smaller signal than B15, as can be seen in Figure 6.15. The only explanation that can be offered for this is that there was a greater time interval (of 
3 days) between the 100 Torr and 20 Torr measurements of B31 and those of B15. These few extra days may have allowed for a greater unrecoverable contamination of the CsI in B31 than in B15.

When the pulse heights (Figure 6.13) and correction factors (Figure 6.14) from the pre-run data sets are examined, the same apparent trends seen in the 100 Torr data are present. Once again the measurements are not spaced evenly in time. The same pump-out and refill procedure used during the 100 Torr measurements was used here after the final data set was collected. Upon refilling with clean $\mathrm{CH}_{4}$ a 500 event data set was collected, and again the pulse heights show an increase over the post-342 $\mathrm{V}$ runs of $\sim 10-20 \%$.

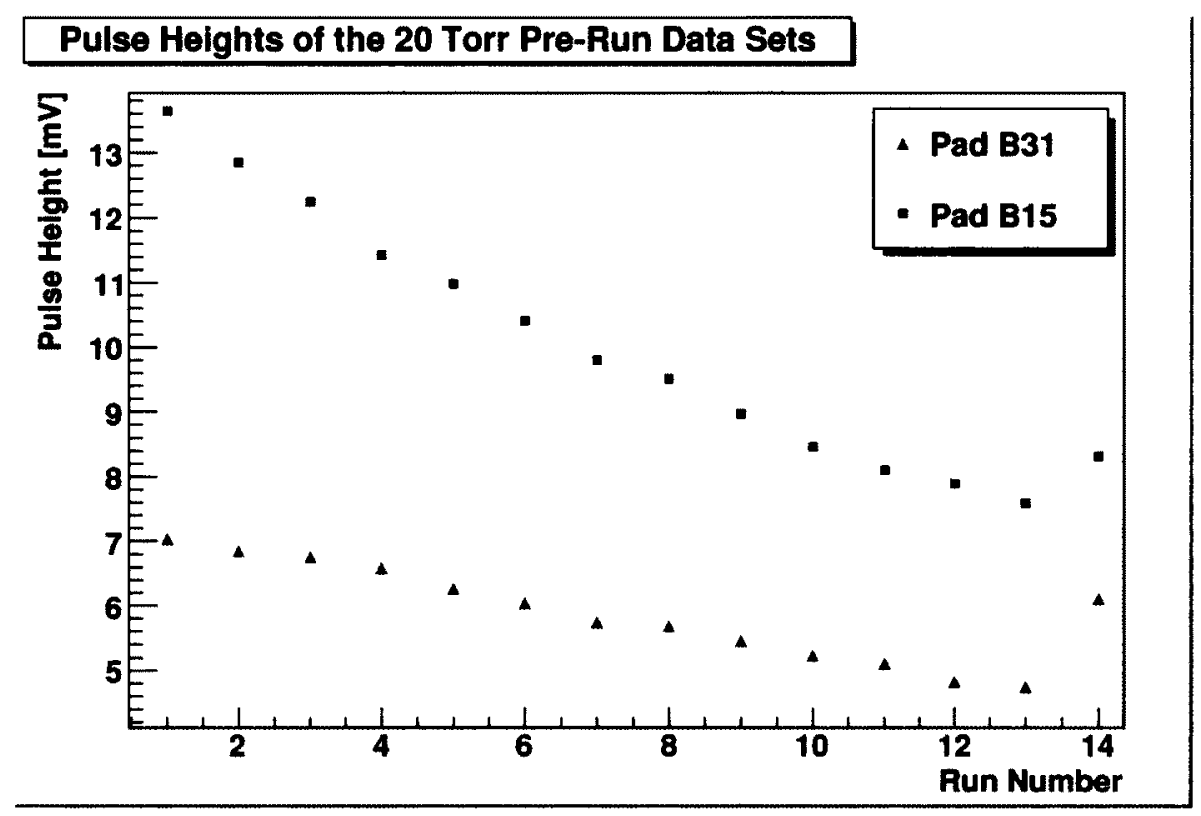

Figure 6.13: Peak heights from the 20 Torr pre-run data sets. The time between points is not constant, though for all but the last point the spacing is roughly 30 minutes. The points for Run 1 for each data set is used as the initial pulse height to correct subsequent runs. The points for Run 13 were taken after the final run at $342 \mathrm{~V}$. The points for Run 14 were taken the following day after the detector package had been evacuated and re-filled with clean $\mathrm{CH}_{4}$. 


\section{Correction Factors From the 20 Torr Pre-Run Data Sets}

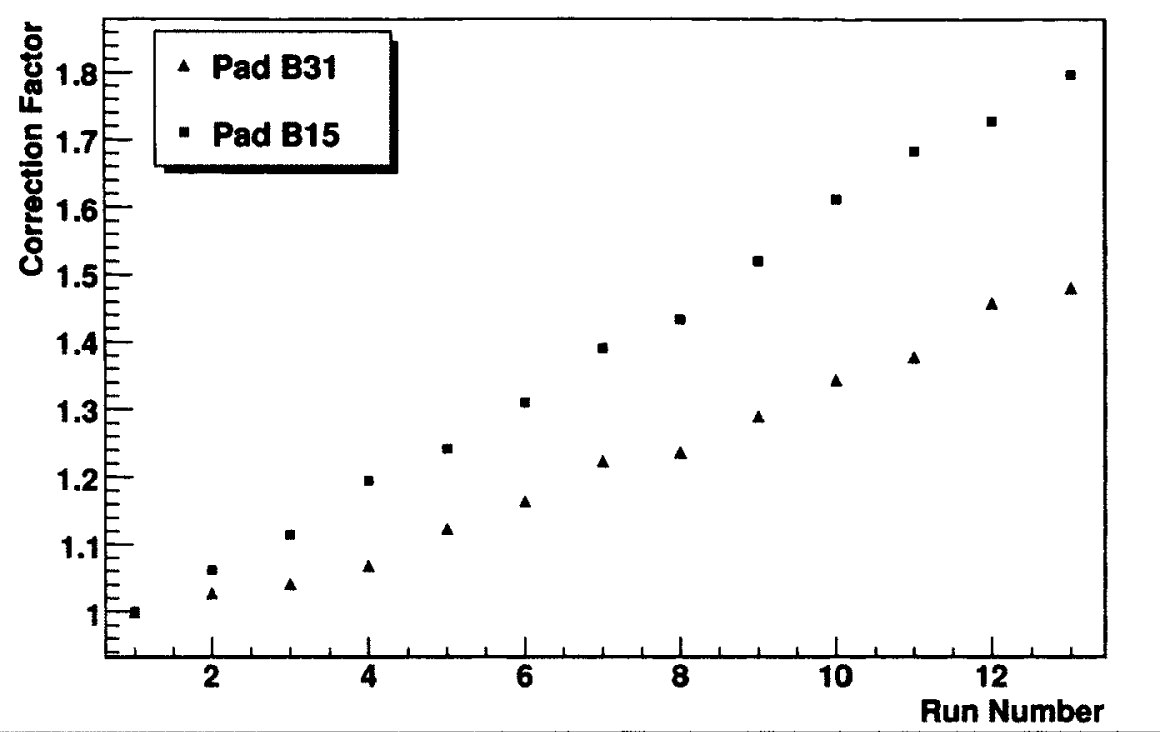

Figure 6.14: Corrections factors from the 20 Torr pre-run data sets. The time between runs is roughly 30 minutes, but is not consistent.

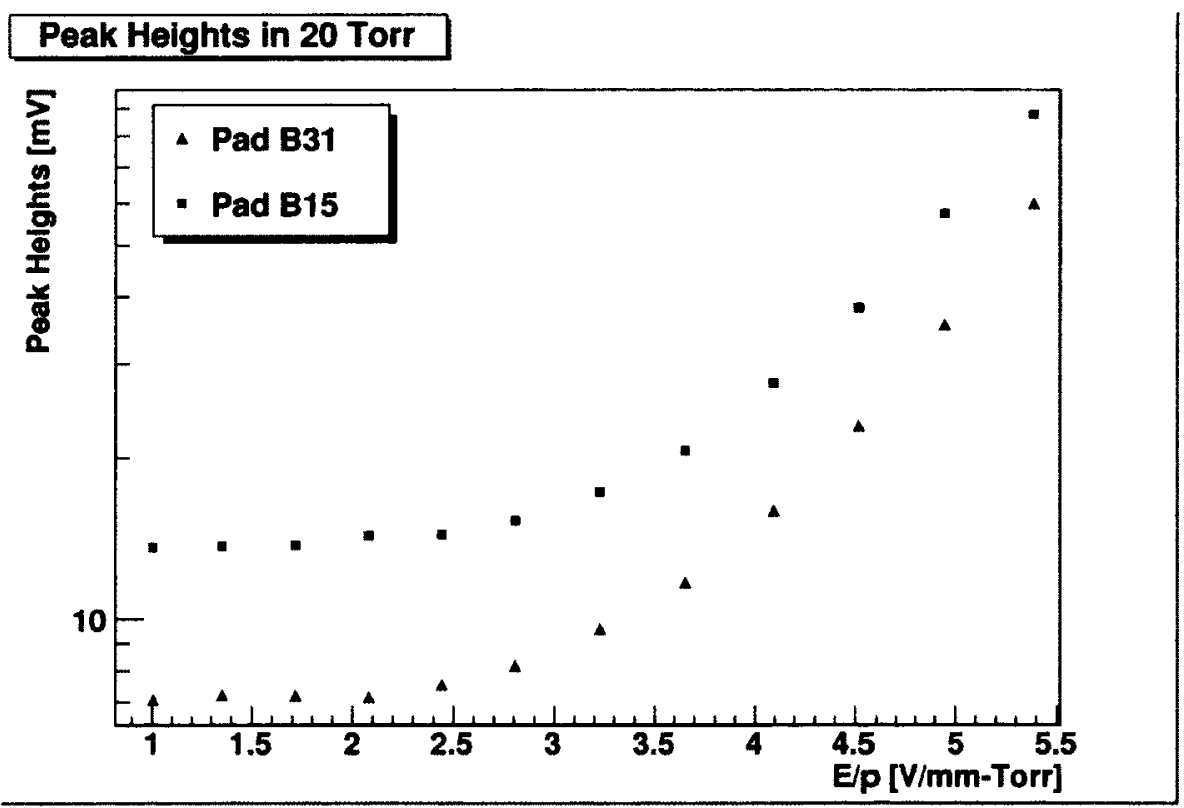

Figure 6.15: Pulse height as a function of $\mathrm{E} / \mathrm{p}$ for 2 pads in 20 Torr. The pulse heights are taken from fits to distributions of the shaped pulse heights, which themselves are fits to individual pulses. The fit errors are too small to be seen at this scale. 
While the relative pulse heights between the two pads has changed since the 100 Torr runs, the earlier onset of gain previously seen on B31 (Figure 6.9) is still present. This is more noticeable in Figure 6.15 than in Figures 6.16 and 6.17 which show the relative signal gains of the two pads.

\section{Torr Gain Curves}

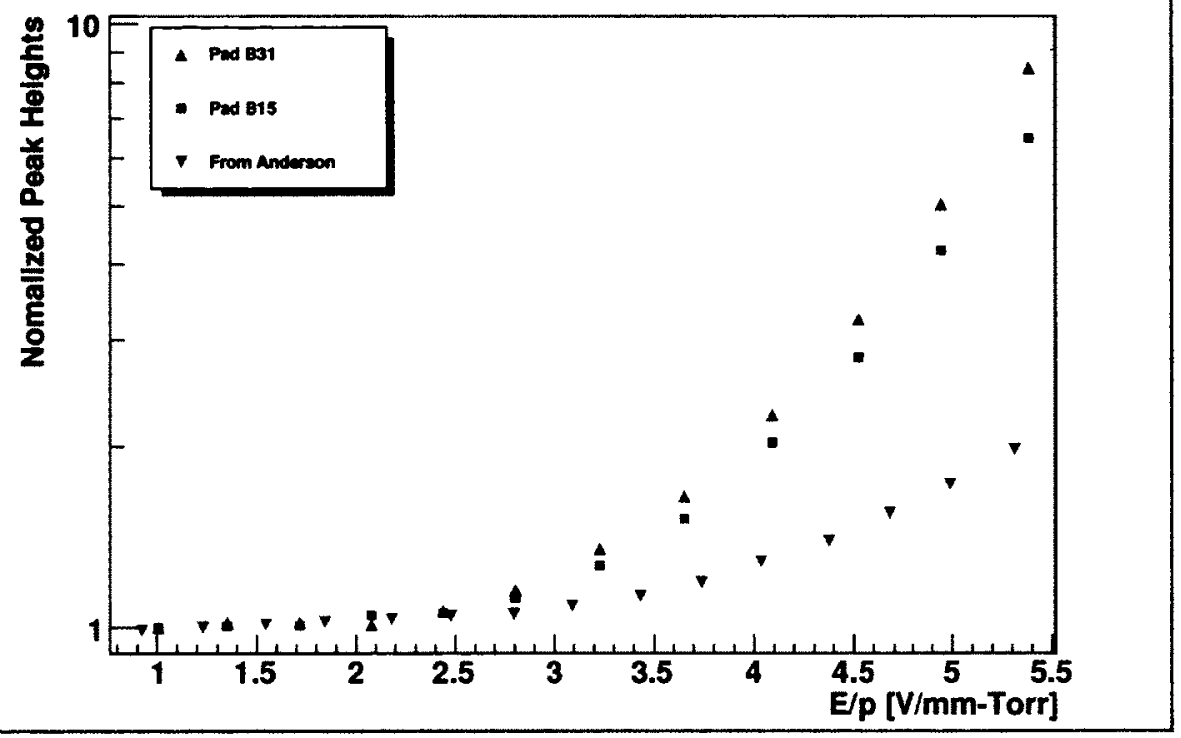

Figure 6.16: The same values from Figure 6.15 are shown, with the pulse heights for each pad given as a ratio to the value at $\mathrm{E} / \mathrm{p}=1.0$ to calculate gain. Also shown are values from Anderson [42] which have been normalized to the value closest to $E / p=1.0$. The fit errors are too small to be seen at this scale. 


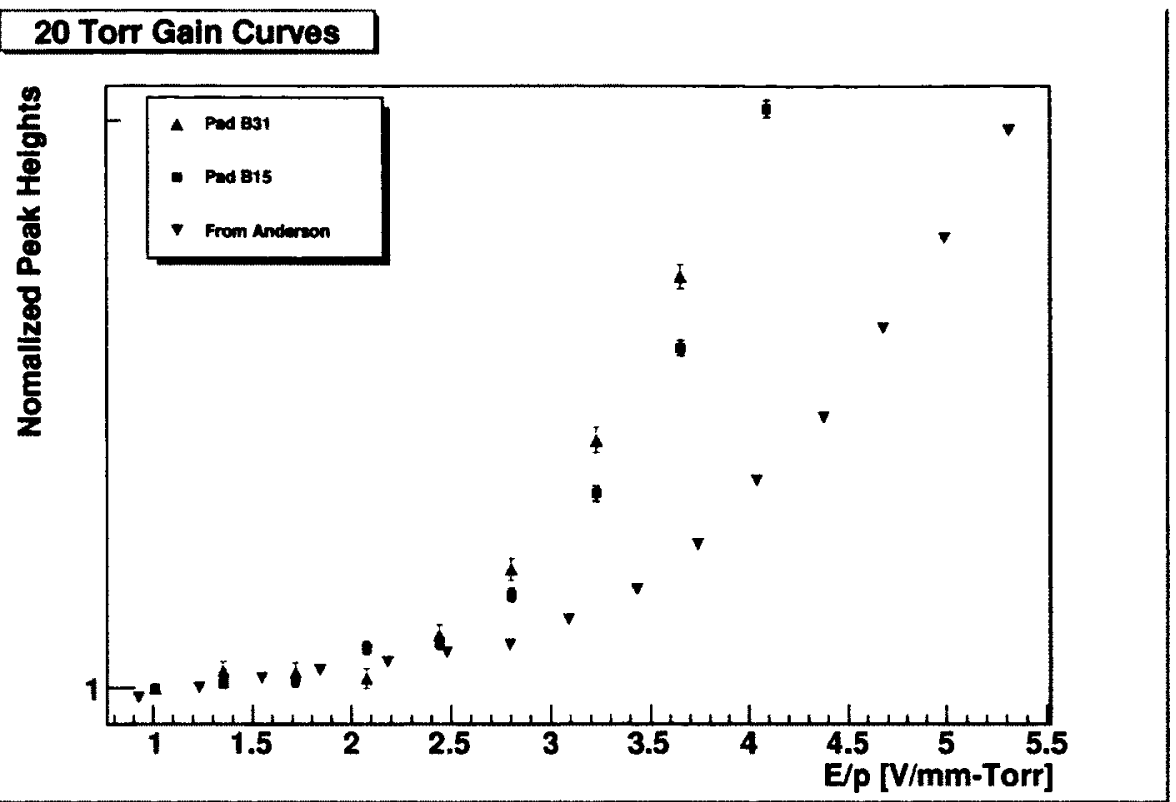

Figure 6.17: The same plot from Figure 6.16 focused to show the no-gain region and the Anderson data [42].

As with the 100 Torr data, the 20 Torr data shows that the first XEP CsI photocathode produces significantly more gain than the Anderson data. 


\section{Pad Resolutions in 20 Torr}

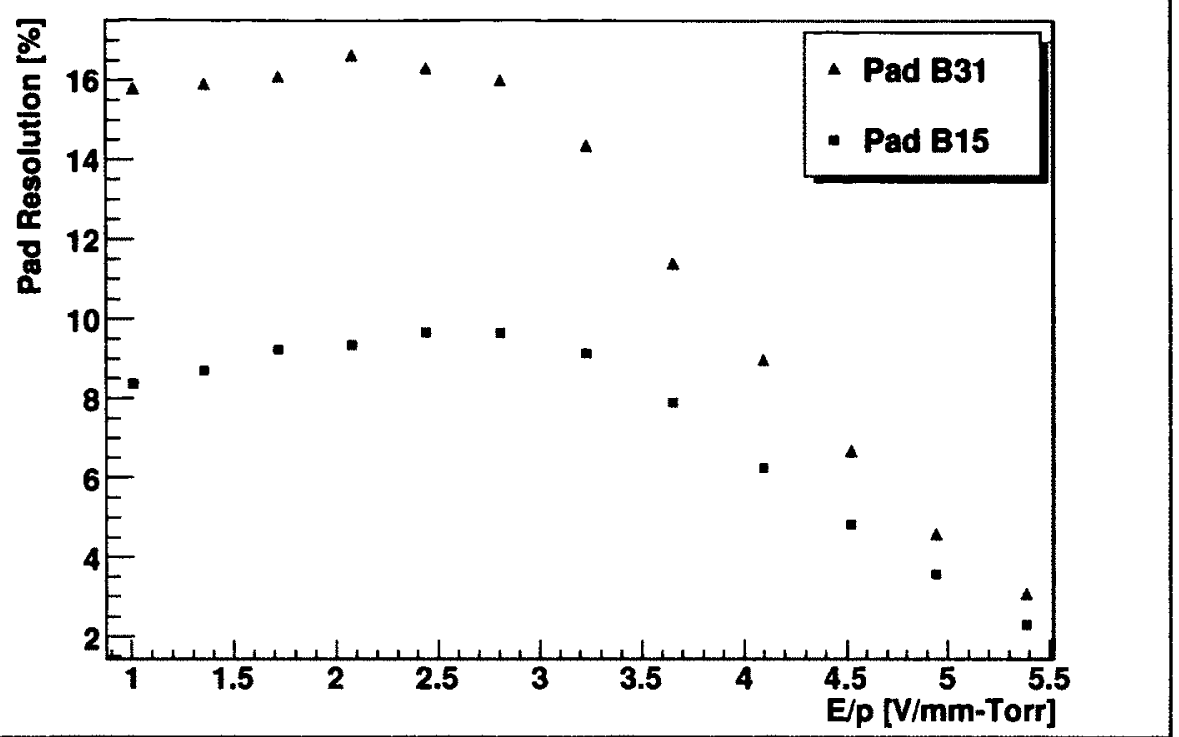

Figure 6.18: The calculated resolution of 2 CsI pads in 20 Torr as a function of $\mathrm{E} / \mathrm{p}$. The fit errors are too small to be seen at this scale.

The same general trends seen in the 100 Torr data are seen in the 20 Torr data with respect to gain and resolution in both the gain and no-gain regions. Again, the reason for the deterioration of the resolution in the no-gain reason is not understood (see Figure 6.18).

\subsection{Pad degradation}

As previously stated, it is apparent that the large amount of UV illuminating the pads may be having a damaging effect on the CsI. To examine this possibility, two long data sets were taken on pad B23, first one at 'low' intensity and then one at 'high' intensity. The data sets were taken over roughly 6 hours, collecting 64000 pulses each. The low intensity data was taken in $\mathrm{CH}_{4}$ at 20 Torr, and at a voltage of $635 \mathrm{~V}(\mathrm{E} / \mathrm{p}$ $=10$ ). The high intensity data was taken at the same pressure, but the voltage had to be reduced to $620 \mathrm{~V}(\mathrm{E} / \mathrm{p}=9.76)$ as the pulses were too large to be read from the 
oscilloscope. The fibre optic cable was not moved between runs, so the area of the pad that was illuminated remained the same.

For each run there was a small upward drift in the voltage as the power supply warmed up. The drift was only $5-6$ volts but at this high $E / p$ there is a noticeable effect on the gain. In practice the power supply has been observed to stabilize after roughly 40 minutes of operation; the first 12800 events of the low intensity run and the first 20000 of the high intensity run, each taken over the first $\sim 1.5-2$ hours, were discarded to remove any effect the power supply warm-up may have had on the pulse heights.

\section{Peak Heights vs. Time of Run}

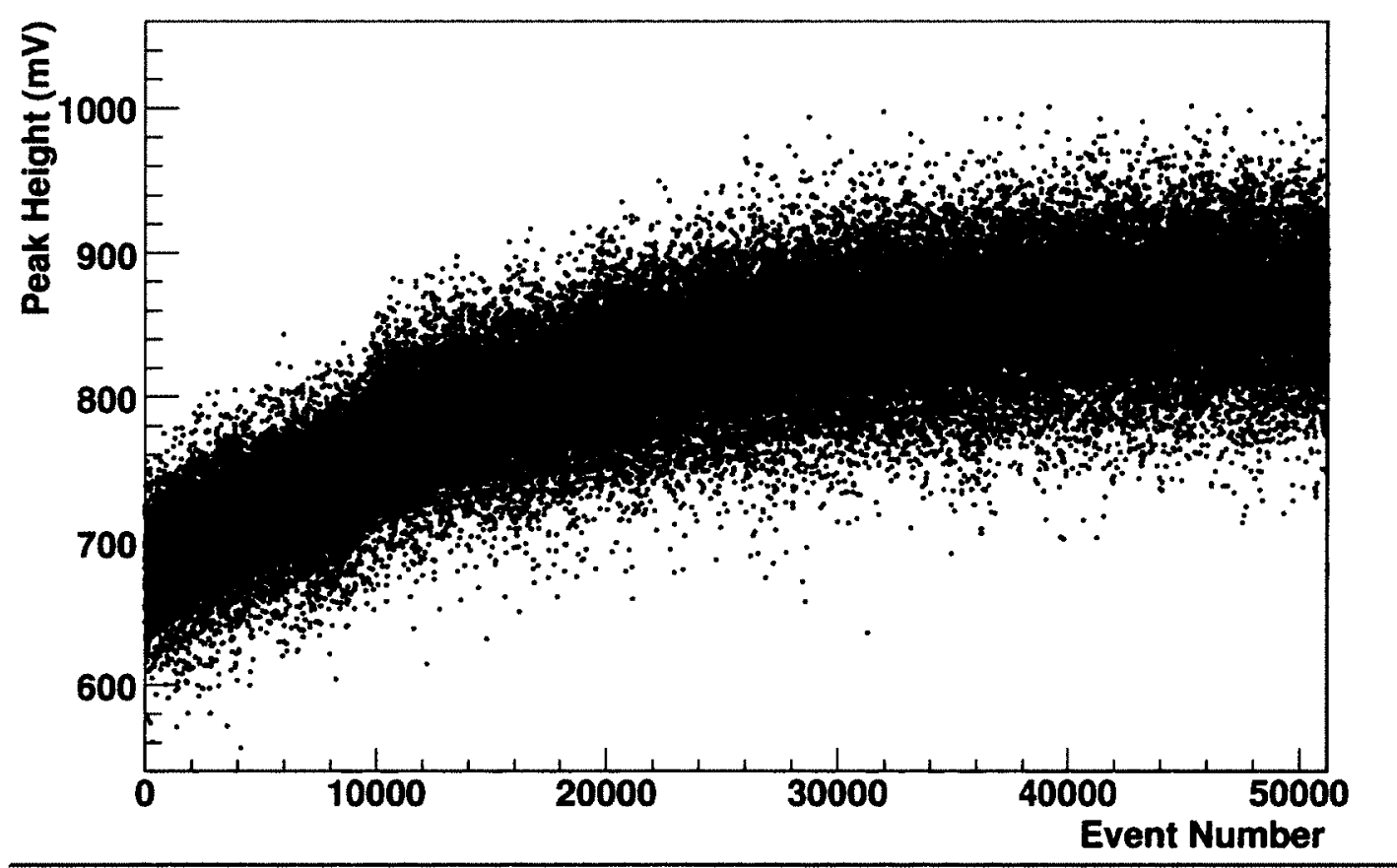

Figure 6.19: Plot showing the drift in peak heights over time for the low intensity run. The length of the run was approximately 4.5 hours.

An interesting effect is seen during the low-intensity run. The peak heights increase over time up to a maximum, then appear to begin decreasing toward the end 
of the run. This is not attributed to warm-up of the power supply and drift of the applied voltage as the events during warm-up were discarded. A similar effect is documented by Anderson [42]; the quantum efficiency can actually increase if the CsI is exposed to VUV in methane over a period of time. Since this pad had not really been examined prior to this test, and hence had not undergone any of this conditioning yet, this explanation seems plausible. The downturn in the pulse heights likely signals a plateau of the quantum efficiency and the point where contamination of the $\mathrm{CH}_{4}$ and UV damage begin to dominate.

One may note that this effect is not present in the data for the gain curves which were previously discussed. The reason for this is that the pads used in those tests, B15, B22, and B31, had been extensively examined prior to the collection of those final data sets. Since there is a limit, over perhaps a few hours to a day, to which this effect can improve the quantum efficiency, it is reasonable to say that these pads had already benefited maximally from it. This serves to further suggest that the plateau and beginning of a downward trend in Figure 6.19 indicate that the limit of the effect was reached during the low intensity run. 


\section{Peak Heights vs. Time of Run}

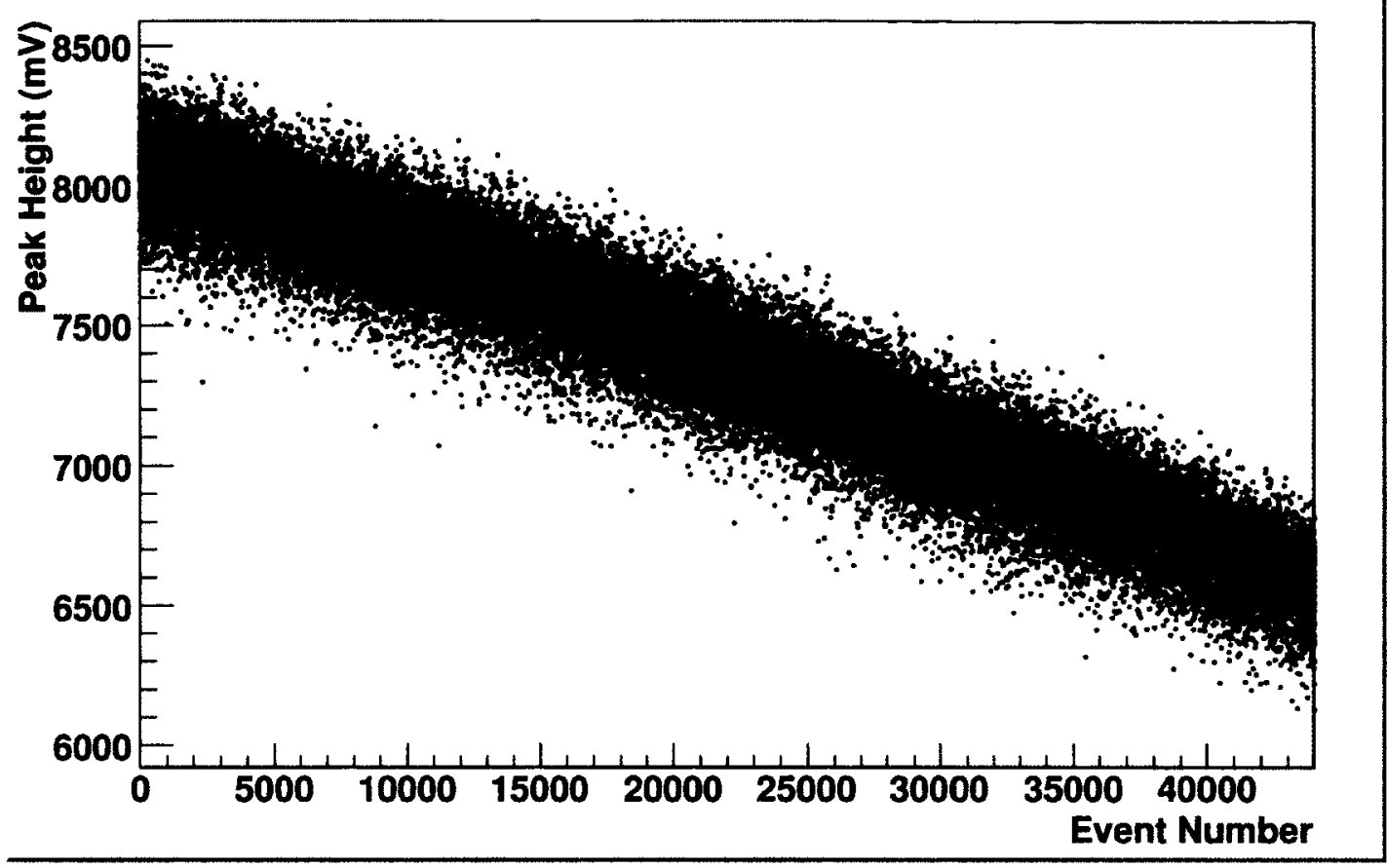

Figure 6.20: Plot showing the drift in peak heights over time for the high intensity run. The length of the run was approximately 4 hours.

Figure 6.20 shows the data from the high intensity run, which was taken after the low intensity run. The degradation of the signal is now quite clear, dropping from $\sim 8100 \mathrm{mV}$ to $\sim 6600 \mathrm{mV}$ - a drop of nearly $20 \%$ over 4 hours. Fortunately, this degradation is unlikely to affect XEP during normal operation. The flash lamp produces significantly more photons per pulse than XEP will see from a $1 \mathrm{MeV}$ electron for example. In addition, the spectral output of the flash lamp is quite broad, peaking above $2000 \mathrm{~nm}$. The scintillation and EL photons have a comparatively narrow spectrum centered at $172 \mathrm{~nm}$. Because of these reasons, any degradation seen during these tests due to UV damage will not be a factor in XEP. 


\subsection{Response across a pad}

For a single pad, the response at different locations on the pad surface were measured. Since the fused silica window scatters the light somewhat, the area on the pad illuminated by the lamp is larger than the diameter of the output from the fibre. The fibre was placed such as to minimize the amount of visible light illuminating the Teflon spacer, thus maximizing the area of the pad that was illuminated. This is of greatest importance near the corners of the pad. Five points were measured on pad C9 from one corner to the opposite corner. Approximate locations of the measurements are shown in Figure 6.21. Each run collected 5000 pulses and was taken at a voltage of $635 \mathrm{~V}$ using the lowest intensity output the lamp could produce. This was done to minimize the effects of UV damage on the pad as discussed in previous sections.

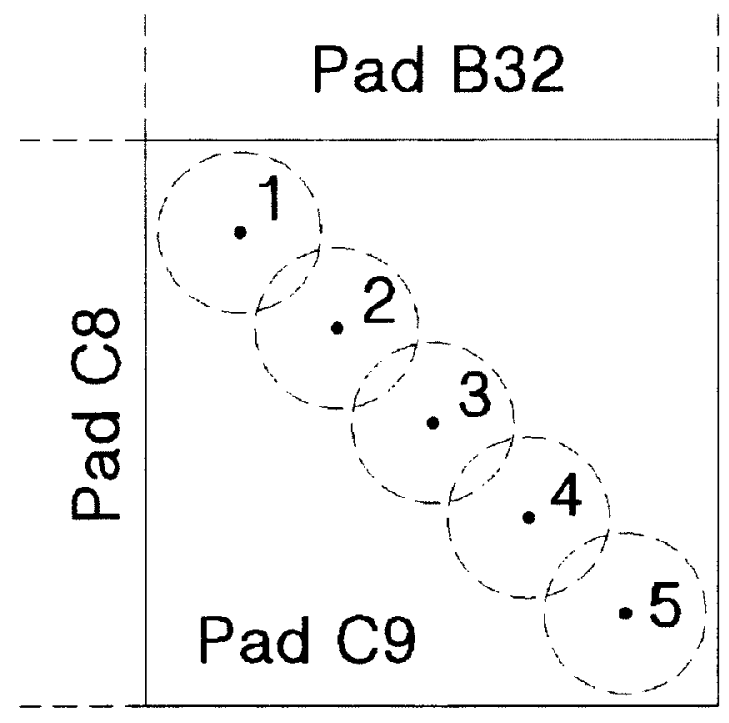

Figure 6.21: The approximate locations of the 5 positions of the fibre for the response across a pad test, in the order they were measured. The small dark circles show the approximate locations and sizes of the light pulses as they exit the fibre. The larger dashed circles show roughly the dispersed visible light as seen on the pad. 


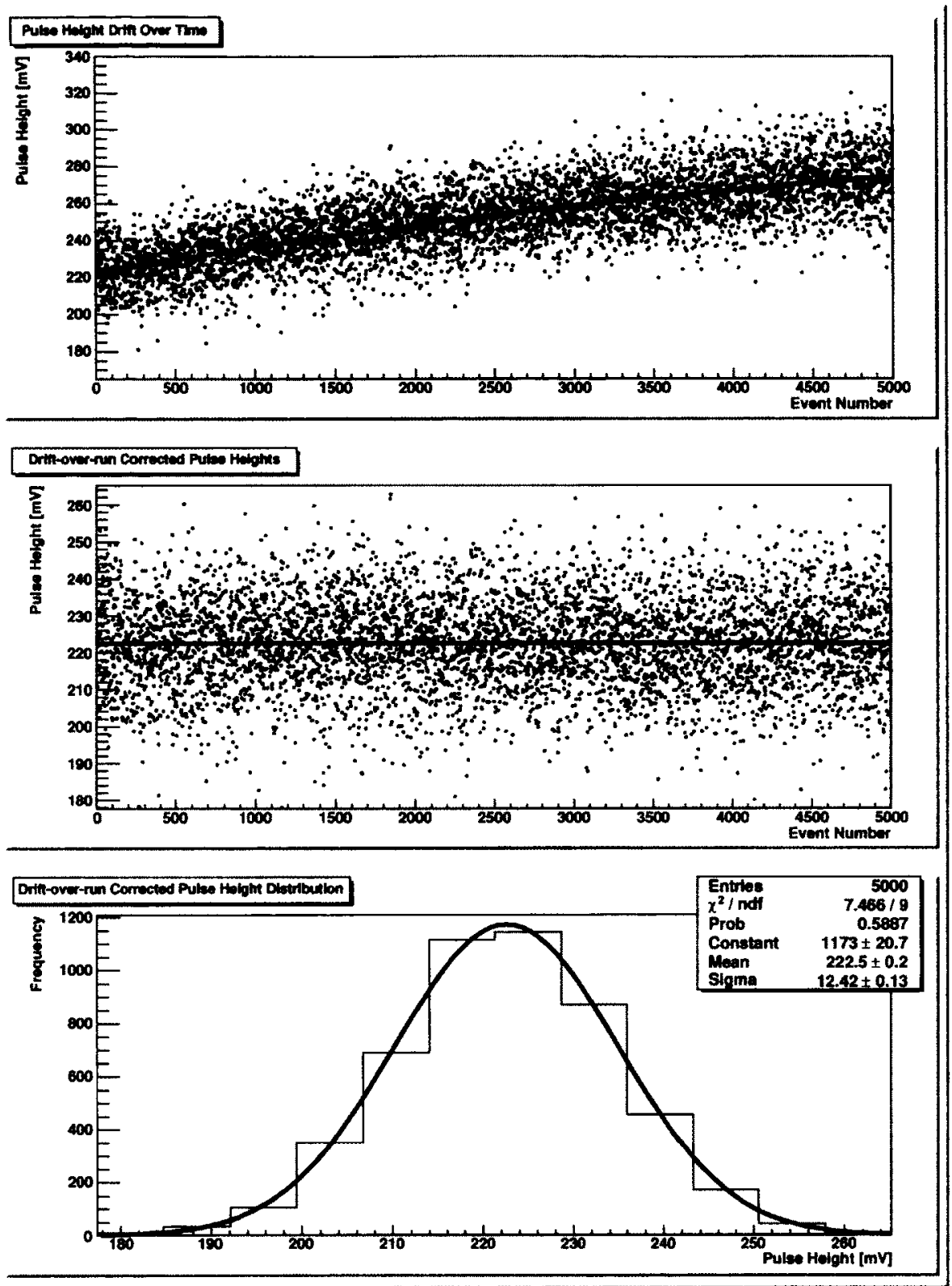

Figure 6.22: Pad-scan data at position 1, showing the original pulse height drift in time and the corrected pulse heights. 


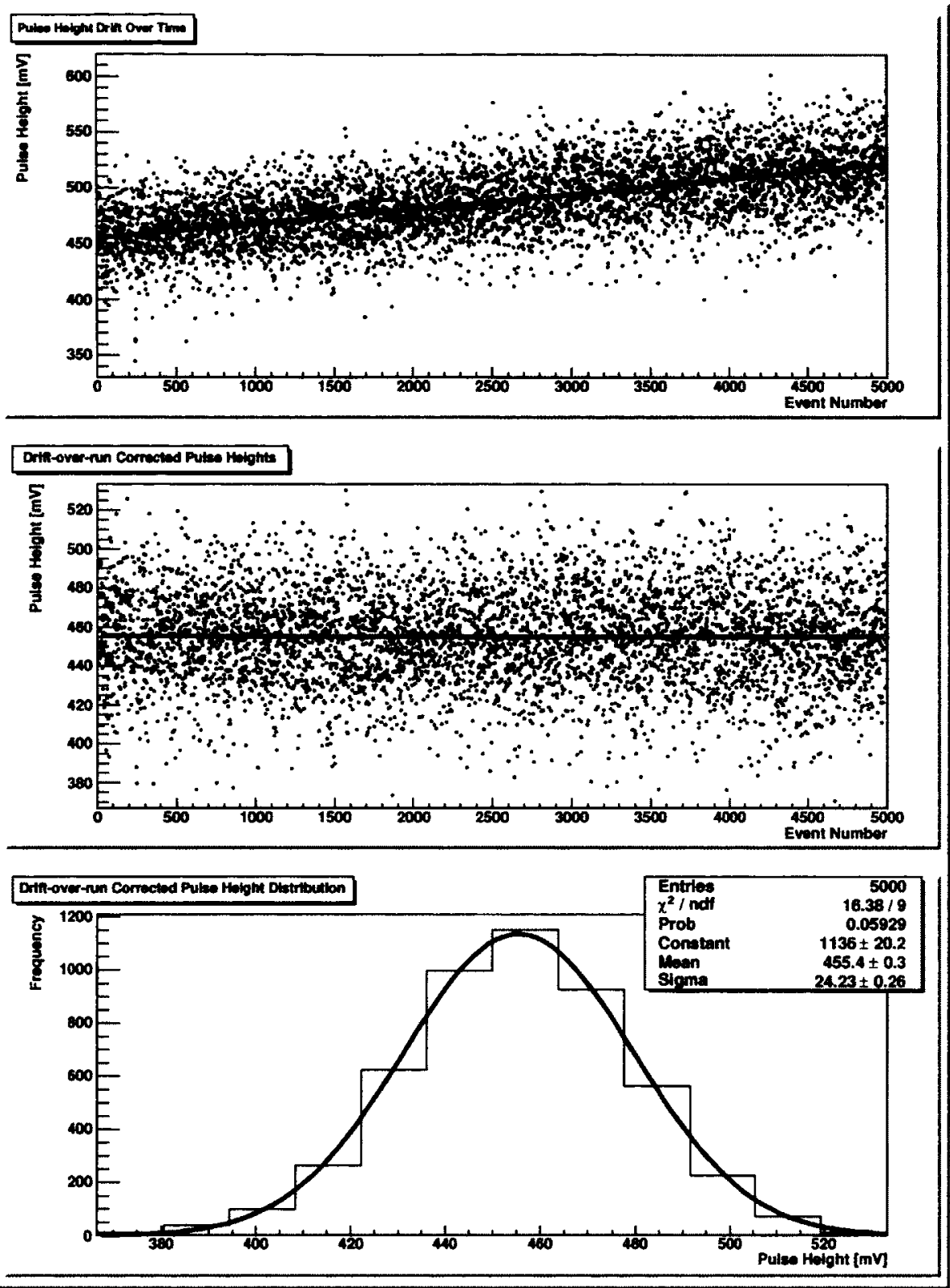

Figure 6.23: Pad-scan data at position 2, showing the original pulse height drift in time and the corrected pulse heights. 


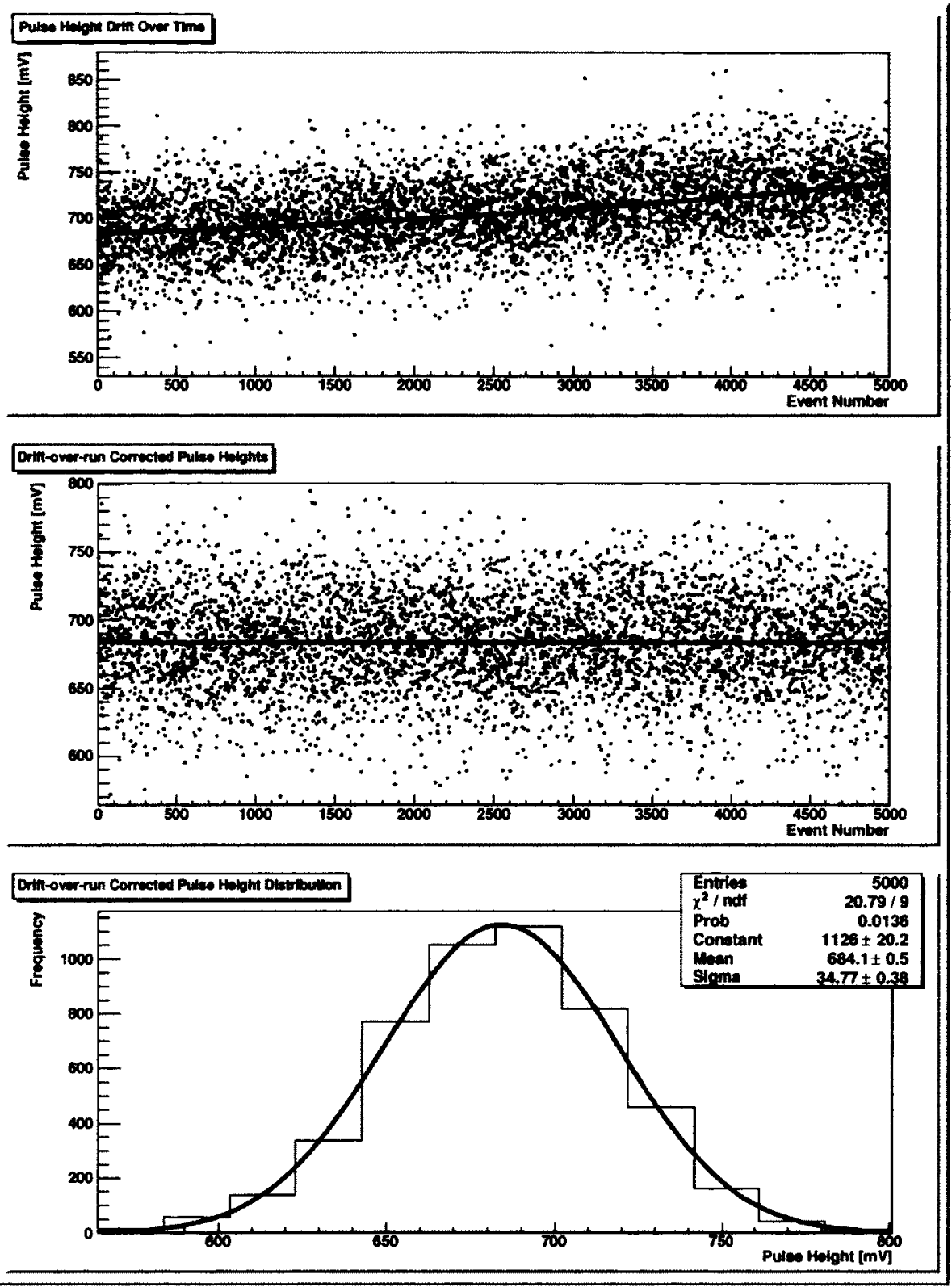

Figure 6.24: Pad-scan data at position 3, showing the original pulse height drift in time and the corrected pulse heights. 


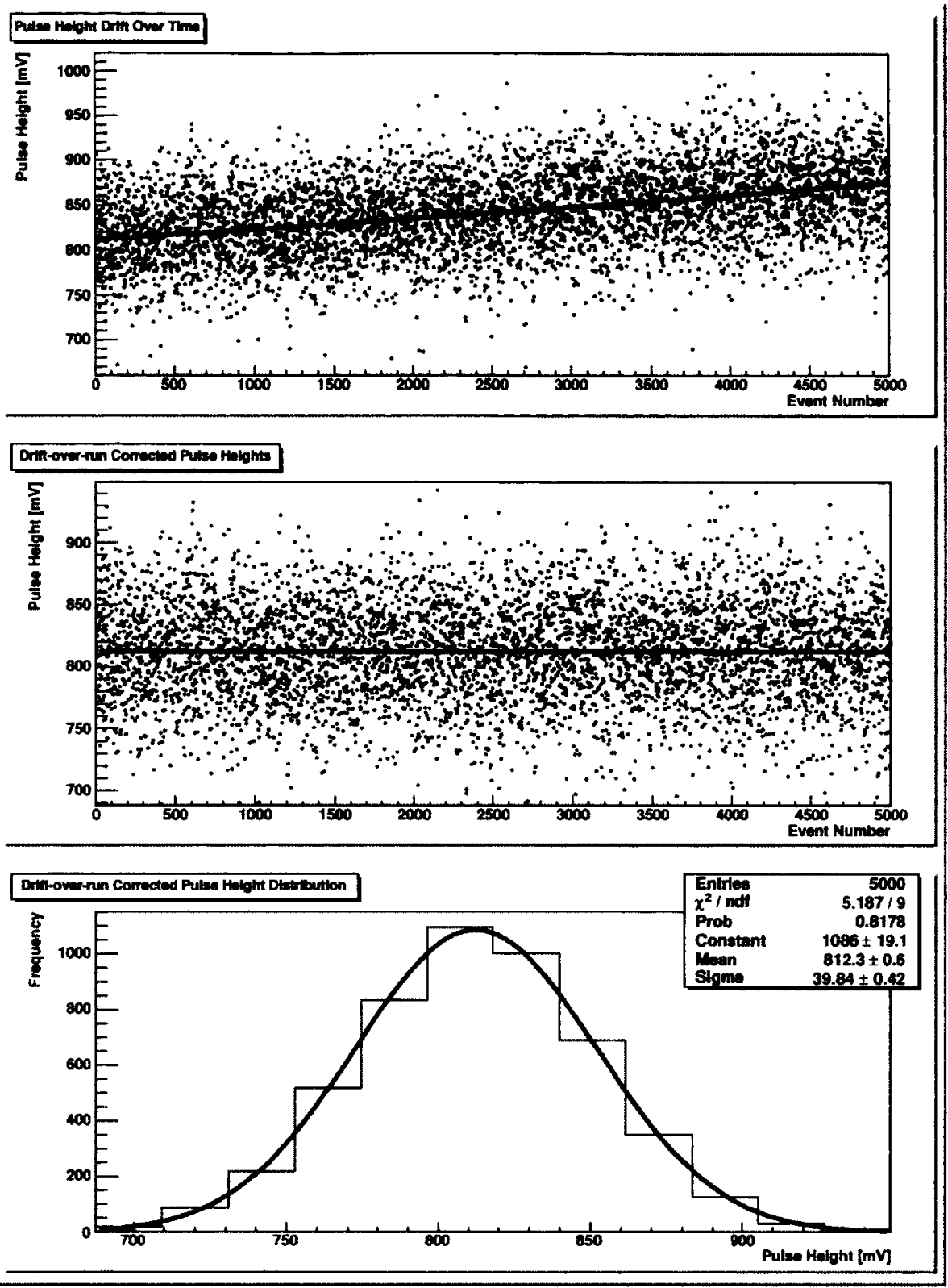

Figure 6.25: Pad-scan data at position 4, showing the original pulse height drift in time and the corrected pulse heights. 


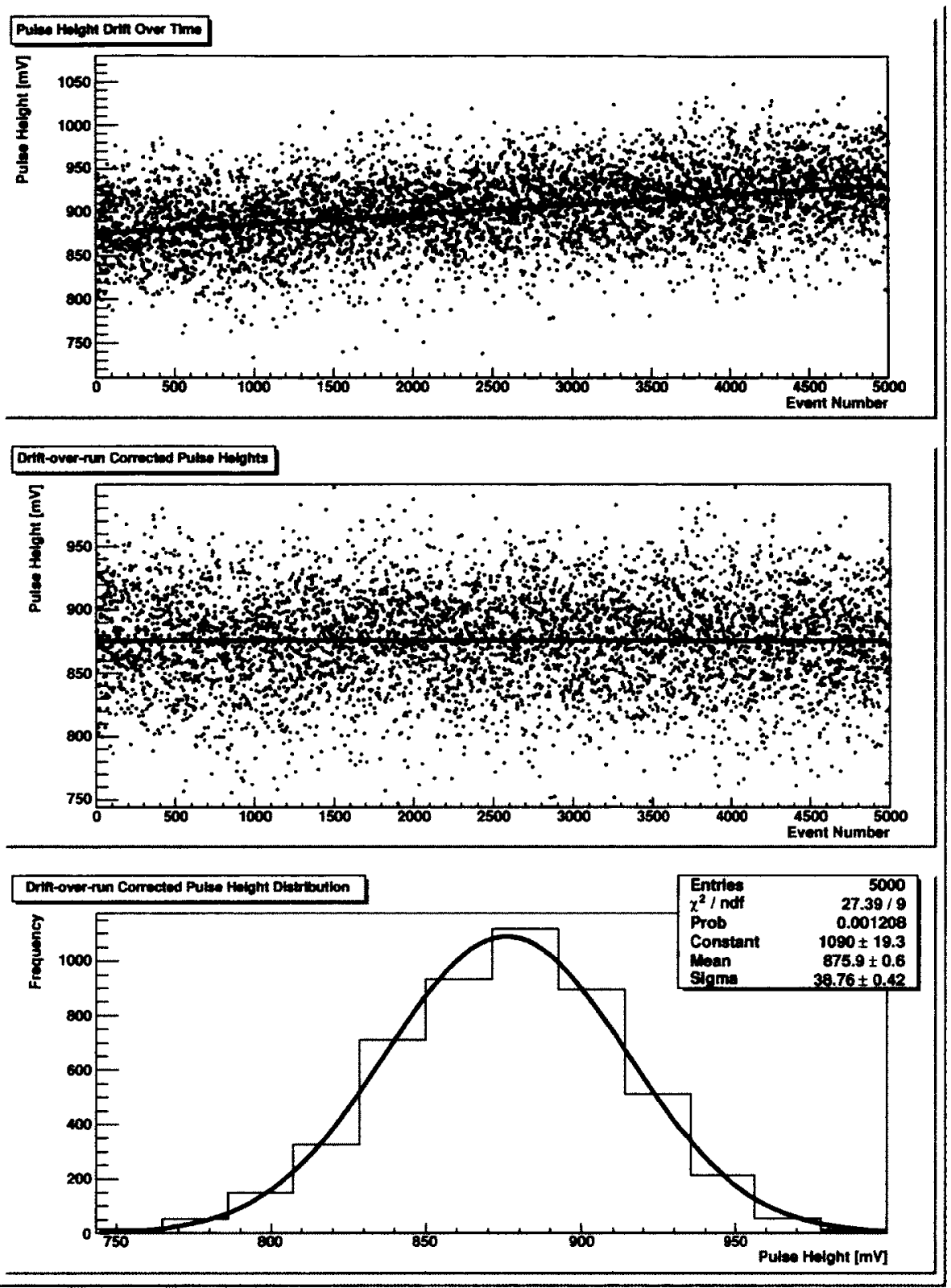

Figure 6.26: Pad-scan data at position 5, showing the original pulse height drift in time and the corrected pulse heights.

Pad C9 is another pad which had not previously been exposed to much UV. As noted in Section 6.2, UV exposure in $\mathrm{CH}_{4}$ can improve the quantum efficiency of the CsI. As in pad B23, this effect is apparent in C9 and can be seen in the pulse height 
distributions for each measurement (Figures 6.22,6.23, 6.24, 6.25, and 6.26). Each of these 5000 event data sets took about 25 minutes to complete, so the entire run took just over 2 hours. When this effect was seen in Figure 6.19 the apparent quantum efficiency increase took place over at least 3.5 hours. If the rate of increase between the two pads is the same, then the 25 minutes each run took place over would not have reached the $\mathrm{QE}$ limit. This is supported by the data, as the pulse heights were increasing to the end of the final run.

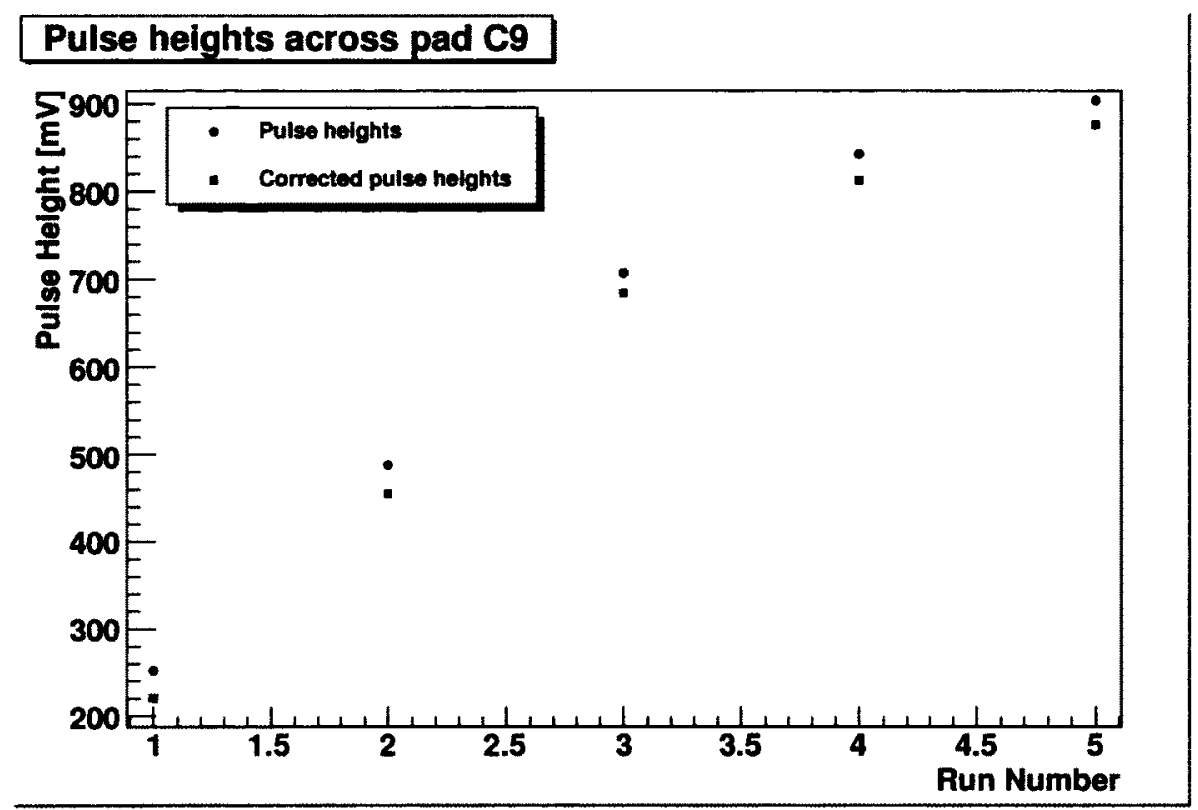

Figure 6.27: Peak heights from 5 locations on a CsI pad. Both the corrected uncorrected values from Figures 6.22, 6.23, 6.24, 6.25, and 6.26 are shown.

In Figure 4.8 a ripple in the mesh beneath pad C9 can be seen. This ripple roughly follows the diagonal path of the measurements that were made, as shown in Figure 6.21 (note that Figure 6.21 is a view looking from the opposite side of the mesh as shown in Figure 4.8). Because of this, variations in pulse height due to a changes in the electric field strength cannot be predicted. With no ripple present, the pulse heights from each of the 5 runs are expected to be the same, so it may be that run-to-run variations may be due to the ripple. 


\subsection{Maximum gain measurement}

The maximum gain that could be achieved before breakdown discharge occurred between the CsI board and the nickel mesh was measured for a single pad in 20 Torr $\mathrm{CH}_{4}$. This test was done in two parts, a 'high' lamp intensity part and a 'low' lamp intensity part. This was done because the pulses (and hence the gain) became too large to be recorded from the scope before breakdown occurred.

The high intensity measurements began in the no-gain region at an E/p of 1 and continued to an $\mathrm{E} / \mathrm{p}$ of 10 in steps of 1 . With a greater electric field than this the shaped pulse was too large to be read from the scope. The low intensity measurements were taken by moving the end of the fibre optic cable about $2 \mathrm{~cm}$ away from the flash lamp. To connect this data to the high intensity data, data was taken for $\mathrm{E} / \mathrm{p}$ values of 9 and 10, and continued to 12. Breakdown in the $\mathrm{CH}_{4}$ region was then observed when the applied voltage was roughly $810 \mathrm{~V}$. The voltage was reduced to $800 \mathrm{~V}(\mathrm{E} / \mathrm{p}$ $=12.6$ ) and an approximate pulse height was read from the scope. However, before data could be taken at this voltage the chamber began to breakdown again, and the voltage at which this effect occurred lowered. For this test, each data set contains 500 pulses. These smaller data sets were taken so that the effects of signal degradation were minimal. The measured pulse heights are shown in Figure 6.28. 


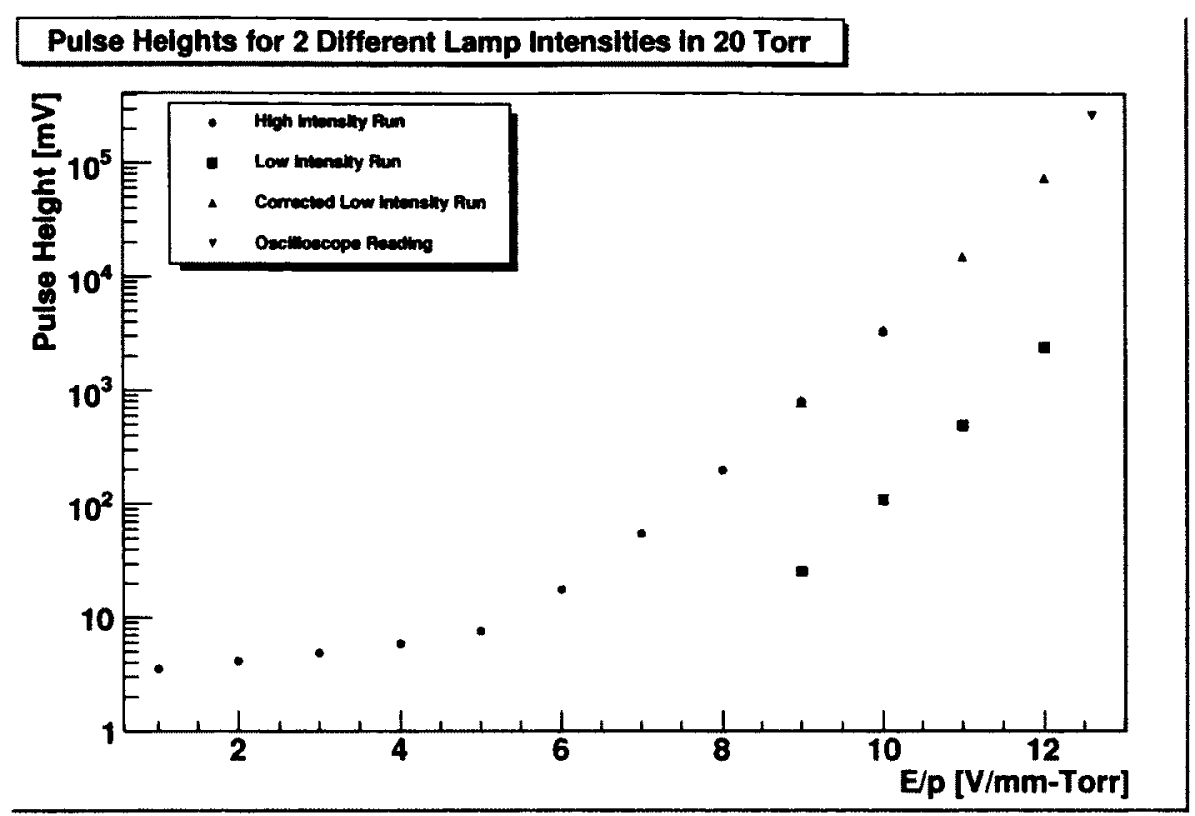

Figure 6.28: Measured pulse heights for pad B14 in 20 Torr methane. The average of the difference between the high and low intensity runs for $E / p$ values of 9 and 10 was used to scale the low intensity data to the high intensity data. The final point in this plot, at $\mathrm{E} / \mathrm{p}=12.6$, does not come from a fit to a distribution of pulse heights, but was rather approximated from the oscilloscope output before breakdown occurred. This point is included to show a possible maximum pulse height.

The gain curve for this test is shown in Figure 6.29. The maximum calculated gain is about 20,000 . This is important as it means that we will easily be able to see signals produced by the relatively lower number of photons in the scintillation signals. Another nice result is that the pad resolution continued to improve with increasing $\mathrm{E} / \mathrm{p}$ during the high intensity run, as seen in Figures 6.30 and 6.31 . For the low intensity run the fibre was moved away from the flash lamp; this negatively affects the resolution. This is because the greater distance through air the photons must travel to enter the fibre introduces larger statistical fluctuations in the number of photons in each pulse. The maximum resolution seen during the high intensity run is $1.75 \%$, and when we consider that the flash lamp has a maximum stability of $1 \%$, the actual pad resolution is more like $\sqrt{1.75^{2}-1^{2}}=1.5 \%$. The 'high' intensity run 
was actually taken with the lamp output at minimum, so the stability is likely worse than $1 \%$. This means that the pad resolution of $1.5 \%$ is an upper limit. This is a very promising first result in terms of the energy resolution goals of XEP.

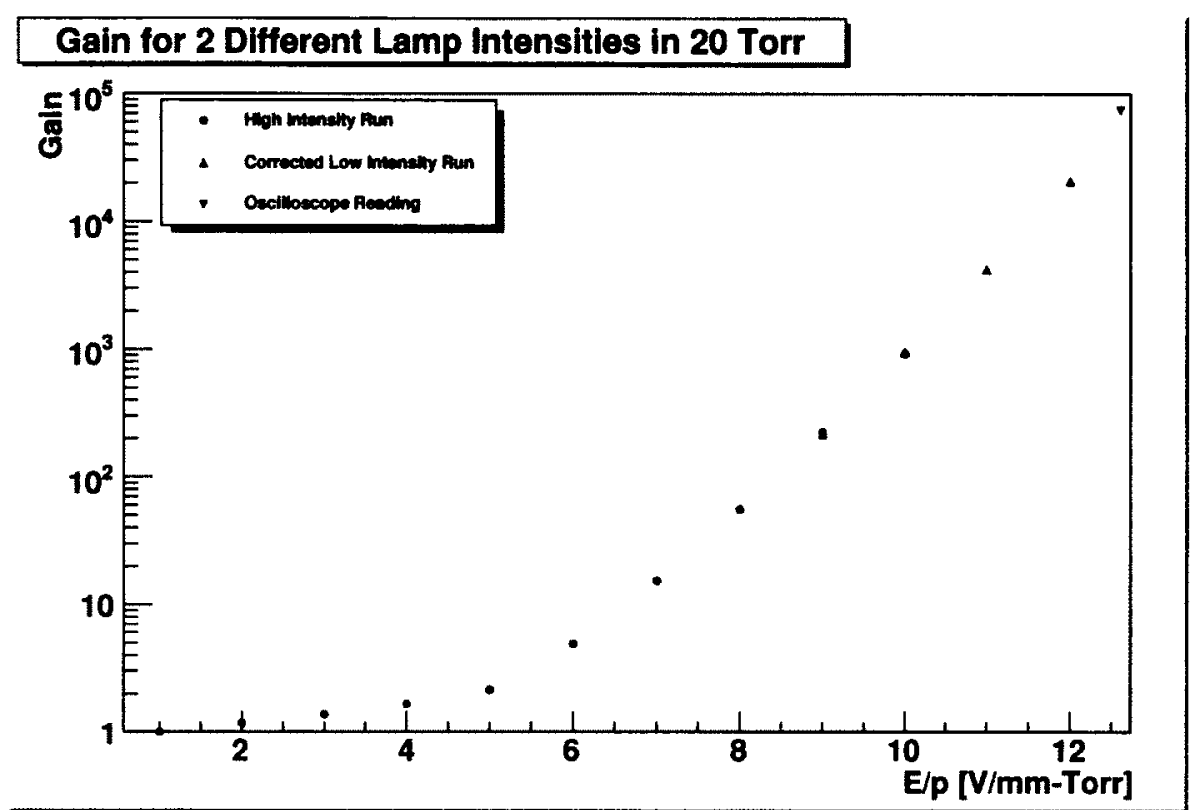

Figure 6.29: A gain curve for pad B14 in 20 Torr methane with all values normalized to the lowest $\mathrm{E} / \mathrm{p}$ value. The final point in this plot, at $\mathrm{E} / \mathrm{p}=12.6$, does not come from a fit to a distribution of pulse heights, but was rather approximated from the oscilloscope output before breakdown occurred. This point is included to show a possible maximum pulse height. 


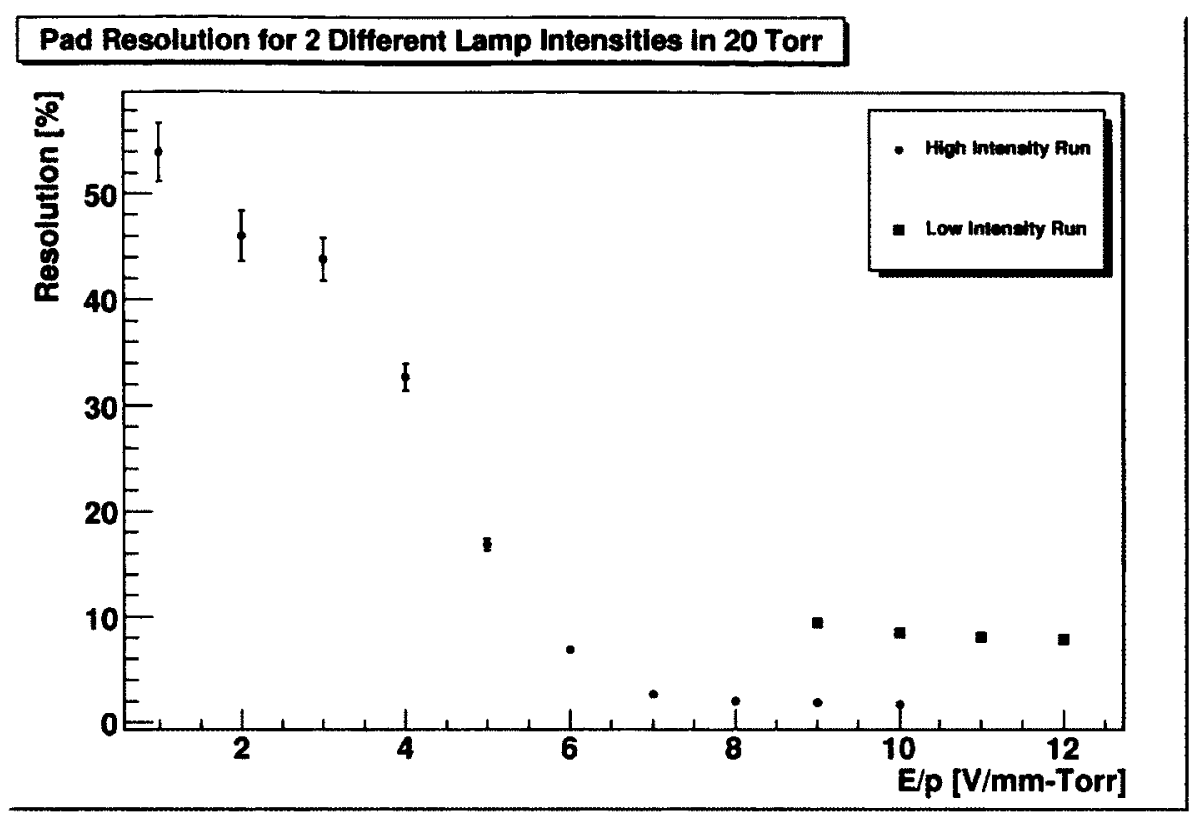

Figure 6.30: The resolution of the pad from the fits to the pulse height distributions taken during the maximum gain test.

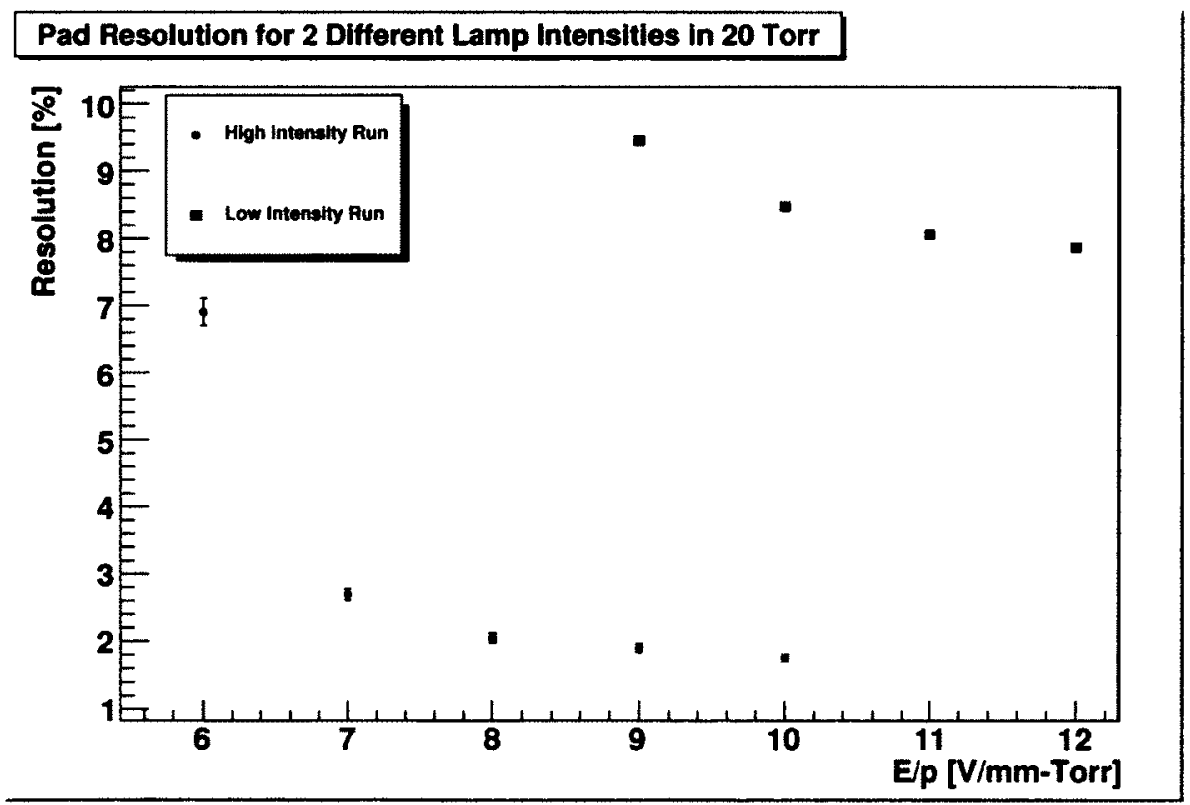

Figure 6.31: The same plot from Figure 6.30 focused to show the better resolution values. 


\section{Chapter 7}

\section{Summary and Conclusion}

The Xenon Electroluminescent Prototype is a project endeavouring to produce a gasphase xenon TPC as part of the Enriched Xenon Observatory. The goal of EXO is the observation and measurement of neutrinoless double beta decay, a process whose halflife can be related to the effective electron neutrino mass. This process, if it occurs, does so over time scales greater than $10^{25}$ years. A detector attempting observation of this process therefore requires both extremely low background levels and good energy resolution at the $\mathrm{Q}$ value. A gas-phase xenon TPC utilizing electroluminescent light has potential for sub 1\% energy resolution, making such a detector a good candidate for a neutrinoless double beta decay search.

A vacuum evaporation chamber was constructed to perform depositions of CsI which creates the photocathodes for XEP's detector packages. It is important that coatings of CsI be uniform to minimize variations in the quantum efficiency of the photocathode. The deposition pattern produced by the chamber was measured and has been shown to be uniform, with variations of $<10 \%$. This corresponds to $<1 \%$ variation in the quantum efficiency (not this work, estimated from [45]), so thickness dependent pad-to-pad variations in response should be minimal.

The technique of photolithography shows potential as a means of applying narrow, finely spaced, and conductive aluminum lines to fused silica. It is clear, though, that 
further research is required to develop the systems that would be needed to apply the process on the $\sim 50 \mathrm{~cm}$ scale of XEP's windows.

The data acquisition electronics to be used during the commissioning of XEP were characterized. This set of electronics consisted of 4 CAEN A2792 modules. A pulse generator was used to send a pulse to each channel of each module individually, and a DC offset added using the internal DACs. The mean and standard deviation of the pre-pulse baselines were measured for each channel. Additionally, the pulse heights were measured and compared to the input pulse height from the generator. The mean and standard deviation of the pulse heights calculated for each channel. The variations in both the baseline values and the pulse heights are roughly 2 ADC channels, corresponding to $<2 \mathrm{mV}$ of noise. From this preliminary testing, as shown in Section 5.1.1, it appears that individual channels will not degrade XEP's energy resolution for sufficiently large pulses.

The first detector package for XEP was constructed and tested. Degradation of the signal was seen over time and was attributed to contamination of the methane. This contamination may be due to outgassing products from interior components such as the Teflon spacer. These contaminants are likely damaging the CsI surface. While not all damage seems to be permanent, there is an unrecoverable decrease in the signal size; that is, to say, unrecoverable without heating the photocathode in vacuum. Anderson et. al. [44] have seen that heating the CsI in vacuum can drive some contaminants off the surface. It is clear, though, that the methane must be circulated and purified to minimize and hopefully eliminate this effect.

Another effect seen is an increase in signal size over the first few hours of a pad's illumination with VUV in methane. This effect has been attributed in the literature to an improvement in the quantum efficiency of the photocathode. This means it may take time for XEP's detector packages to achieve maximum response as they are operated, absent any intentional UV conditioning. 
It has been shown that gain on the order of $10^{4}$ in the methane gap can be easily achieved. It has also been shown that the photocathode resolution, and hence energy resolution of XEP, improves when gain is applied. The best resolution seen, considering the output stability of the flash lamp, is at least $1.5 \%$. These results are an encouraging first step toward the potential demonstration of sub $1 \%$ energy resolution with XEP.

\subsection{Next steps}

Now that testing of the first detector package is complete, there are several steps which can be taken to move XEP toward becoming a working TPC. First of all, some additions should be made to the production and operation of the photocathode with respect to heating. During production, the photocathode surface should be heated in vacuum for an extended period of time - perhaps a couple of days - as this should drive off contaminants which would effect the quantum efficiency. Post-production, the photocathode should again be heated in vacuum to drive off any contaminants which may have accumulated on the CsI during its brief exposure to air. Finally, during normal operation the photocathode should be periodically heated to drive off any contaminants which may have come from internal components of the detector package.

It may be beneficial to XEP to develop a system to perform UV conditioning of the photocathode to improve its quantum efficiency. This could be achieved quite easily with the existing set-up. The detector package with new photocathode could be placed on the dark box with the flash lamp below it. The flash lamp can be set up to illuminate all pads at once; this in conjunction with methane and gain in the chamber may improve the quantum efficiency over approximately a six hour period. Though this is a somewhat simple set-up, Anderson et. al. have seen an increases in 
quantum efficiency of $20 \%$, meaning this could be of significant benefit to XEP.

The set of CAEN modules which have been characterized make up only $1 / 4$ of the total set. Characterization must be completed on all 512 channels from the 16 modules before XEP can run in its ultimate configuration. All channel-to-channel variations must be accounted for, as this will be necessary to determine and optimize the energy resolution of XEP.

We are almost ready to produce a new CsI photocathode and install the detector package on the TPC. To avoid variations in the response caused by ripples in the nickel mesh, we must replace this component with a more rigid mesh. We must also install the proposed methane flow and purification system to minimize degradation of the CsI pads. With all the aforementioned steps complete, we will be able to begin the commissioning work of XEP and will be in a position to determine XEP's ultimate energy resolution. 


\section{References}

[1] F. Reines and C. L. Cowan, Detection of the Free Neutrino, Phys. Rev. 92, 830 (1953).

[2] G. Danby et al., Observation of High-Energy Neutrino Reactions and the Existence of Two Kinds of Neutrinos, Phys. Rev. Lett. 9, 36 (1962).

[3] Y. Fukuda et al., Evidence for Oscillation of Atmospheric Neutrinos, Phys. Rev. Lett. 81, 1562 (1998).

[4] Q. Ahmad et al., Direct evidence for neutrino flavor transformation from neutral current interactions in the Sudbury Neutrino Observatory, Phys.Rev.Lett. 89, 011301 (2002).

[5] M. Auger et al., The EXO-200 detector, part I: Detector design and construction, JINST 7, P05010 (2012).

[6] S. Glashow, Partial Symmetries of Weak Interactions, Nucl.Phys. 22, 579 (1961).

[7] S. Weinberg, A Model of Leptons, Phys.Rev.Lett. 19, 1264 (1967).

[8] S. Herb et al., Observation of a Dimuon Resonance at 9.5-GeV in 400-GeV Proton-Nucleus Collisions, Phys.Rev.Lett. 39, 252 (1977).

[9] F. Abe et al., Observation of top quark production in $\vec{p} p$ collisionsPhys.Rev.Lett. 74, 2626 (1995).

[10] K. Kodama et al., Observation of tau neutrino interactions, Phys.Lett. B504, 218 (2001).

[11] R. Davis, D. S. Harmer, and K. C. Hoffman, Search for Neutrinos from the Sun, Phys. Rev. Lett. 20, 1205 (1968).

[12] K. Nakamura et al., Review of Particle Physics, Journal of Physics G (2010). 
[13] D. Griffiths, Introduction to Elementary Particles, 2 ed. (WILEY-VCH, Weinheim, 2008), pp. 390-391.

[14] E. K. Akhmedov, Neutrino physics, PARTICLE PHYSICS 1999: Proceedings. Edited by G. Senjanovic and A.Yu. Smirnov. Singapore, World Scientific, 2000. 394p. (ICTP Series in Theoretical Physics, Vol. 16) (1999).

[15] F. An et al., Observation of electron-antineutrino disappearance at Daya Bay, Phys.Rev.Lett. 108, 171803 (2012).

[16] B. Aharmim et al., Combined Analysis of all Three Phases of Solar Neutrino Data from the Sudbury Neutrino Observatory, arXiv 1109.0763 (2011).

[17] P. Adamson et al., An improved measurement of muon antineutrino disappearance in MINOS, Phys.Rev.Lett. 108, 191801 (2012).

[18] R. Barate et al., An Upper limit on the tau-neutrino mass from three-prong and five-prong tau decays, Eur.Phys.J. C2, 395 (1998).

[19] K. Assamagan et al., Upper limit of the muon-neutrino mass and charged pion mass from momentum analysis of a surface muon beam, Phys.Rev. D53, 6065 (1996).

[20] F. Fraenkle, KATRIN: an experiment to determine the neutrino mass, DPF 2011 Meeting of the Division of Particles and Fields of the American Physical Society. 9-13 Aug 2011. Providence, Rhode Island, USA, arXiv 1110.0087 (2011).

[21] C. Kraus et al., Final results from phase II of the Mainz neutrino mass search in tritium beta decay, Eur.Phys.J. C40, 447 (2005).

[22] Avignone, Frank T. , III and Elliott, Steven R. and Engel, Jonathan, Double Beta Decay, Majorana Neutrinos, and Neutrino Mass, Rev. Mod. Phys. 80, 481 (2008).

[23] M. Auger et al, Search for Neutrinoless Double-Beta Decay in ${ }^{136} \mathrm{Xe}$ with EXO200, Phys. Rev. Lett. 109, 032505 (2012).

[24] J. Beringer et al. (Particle Data Group), Review of Particle Physics, Phys. Rev. D86, (2012).

[25] A. Barabash, Average and recommended half-life values for two neutrino double beta decay: Upgrade'05, Czech.J.Phys. 56, 437 (2006). 
[26] N. Ackerman et al., Observation of Two-Neutrino Double-Beta Decay in ${ }^{136}$ Xe with EXO-200, Phys.Rev.Lett. 107, 212501 (2011).

[27] T. K.-Z. Collaboration, Measurement of the double- $\beta$ decay half-life of ${ }^{136}$ Xe with the KamLAND-Zen experiment, Phys.Rev. C85, 045504 (2012).

[28] H. Klapdor-Kleingrothaus and I. Krivosheina, The evidence for the observation of Onu beta beta decay: The identification of Onu beta beta events from the full spectra, Mod.Phys.Lett. A21, 1547 (2006).

[29] P. Vogel, Neutrinoless double beta decay, PROCEEDINGS: Theoretical Advanced Summer Institute TASI-2006. 4-30 Jun 2006. Boulder, Colorado, Edited by S. Dawson, R.N. Mohapatra. Hackensack, World Scientific, 2008. 704p. .

[30] R. Bernabei, Results on Dark Matter and beta beta decay modes by DAMA at Gran Sasso, PROCEEDINGS: Neutrinoless Double Beta Decay (NDBD07), Ahmedabad (India), February 2007, Published in *Kota, V.K.B. (ed.) et al.: Neutrinoless double beta decay* 121-134 (2007).

[31] M. Redshaw, E. Wingfield, J. McDaniel, and E. Myers, Mass and double-betadecay $Q$ value of $X e-136$, Phys.Rev.Lett. 98, 053003 (2007).

[32] T. R. Rodriguez and G. Martinez-Pinedo, Energy density functional study of nuclear matrix elements for neutrinoless $\beta \beta$ decay, Phys.Rev.Lett. 105, 252503 (2010).

[33] J. Menendez, A. Poves, E. Caurier, and F. Nowacki, Disassembling the Nuclear Matrix Elements of the Neutrinoless beta beta Decay, Nucl.Phys. A818, 139 (2009).

[34] J. Barea and F. Iachello, Neutrinoless double-beta decay in the microscopic interacting boson model, Phys.Rev. C79, 044301 (2009).

[35] F. Simkovic et al., The 0 nu bb-decay nuclear matrix elements with self-consistent short-range correlations, Phys.Rev. C79, 055501 (2009).

[36] A. Staudt, K. Muto, and H. Klapdor-Kleingrothaus, Calculation of two neutrino and zero neutrino double beta decay rates, Europhys.Lett. 13, 31 (1990).

[37] E. Aprile, A. Bolotnikov, A. Bolozdynya, and T. Doke, Noble Gas Detectors (WILEY-VCH, Weinheim, 2006), p. 57. 
[38] C. Monteiro et al., Secondary Scintillation Yield in Pure Xenon, JINST 2, P05001 (2007).

[39] C. Oliveira et al., Simulation of VUV electroluminescence in micropattern gaseous detectors: the case of GEM and MHSP, arXiv 1206.1646 (2012).

[40] J. Escada et al., A Monte Carlo study of the fluctuations in Xe electroluminescence yield: pure $\mathrm{Xe}$ vs $\mathrm{Xe}$ doped with $\mathrm{CH}_{4}$ or $\mathrm{CF}_{4}$ and planar vs cylindrical geometries, JINST 6, P08006 (2011).

[41] D. Nygren, Can the 'intrinsic' energy resolution in xenon be surpassed?, J.Phys.Conf.Ser. 309, 012006 (2011).

[42] D. Anderson, S. Kwan, and V. Peskov, CsI and some new photocathodes, Nucl.Instrum.Meth. A343, 109 (1994).

[43] J. Seguinot et al., Reflective UV photocathodes with gas phase electron extraction: Solid, liquid, and adsorbed thin films, Nucl.Instrum.Meth. A297, 133 (1990).

[44] D. Anderson, S. Kwan, and V. Peskov, CsI photocathode QE and a simple production technique, Nucl.Instrum.Meth. A326, 611 (1993).

[45] F. Cusanno et al., Quantum efficiency measurement system for large area CsI photodetectors, Nucl.Instrum.Meth. A502, 251 (2003). 


\section{Appendix A}

\section{Data Tables}

\begin{tabular}{ccccccc}
\hline Voltage & & $\begin{array}{c}\text { Pulse } \\
\text { Height } \\
{[\mathrm{mV}]}\end{array}$ & $\begin{array}{c}\text { Drift-over-run } \\
\text { Correction } \\
{[\mathrm{mV}]}\end{array}$ & $\begin{array}{c}\text { Correction } \\
\text { Factor }\end{array}$ & $\begin{array}{c}\text { Shifted } \\
\text { Pulse } \\
\text { Height[mV] }\end{array}$ & $\begin{array}{c}\text { Shifted } \\
\text { Pulse } \\
\text { Width[mV] }\end{array}$ \\
\hline \hline 254 & 0.80 & $16.59 \pm 0.02$ & $17.07 \pm 0.02$ & 1 & $17.07 \pm 0.02$ & $1.24 \pm 0.01$ \\
330 & 1.04 & $15.97 \pm 0.02$ & $16.20 \pm 0.02$ & $1.064 \pm 0.005$ & $17.24 \pm 0.10$ & $1.28 \pm 0.01$ \\
406 & 1.28 & $15.37 \pm 0.02$ & $15.75 \pm 0.02$ & $1.119 \pm 0.006$ & $17.62 \pm 0.10$ & $1.39 \pm 0.02$ \\
483 & 1.52 & $14.86 \pm 0.02$ & $15.10 \pm 0.02$ & $1.176 \pm 0.006$ & $17.76 \pm 0.11$ & $1.46 \pm 0.02$ \\
559 & 1.76 & $14.43 \pm 0.02$ & $14.70 \pm 0.02$ & $1.227 \pm 0.007$ & $18.04 \pm 0.12$ & $1.53 \pm 0.02$ \\
635 & 2.00 & $14.15 \pm 0.02$ & $14.34 \pm 0.02$ & $1.269 \pm 0.007$ & $18.20 \pm 0.13$ & $1.57 \pm 0.03$ \\
714 & 2.25 & $14.37 \pm 0.02$ & $14.60 \pm 0.02$ & $1.331 \pm 0.008$ & $19.43 \pm 0.16$ & $1.63 \pm 0.03$ \\
794 & 2.50 & $15.60 \pm 0.02$ & $15.70 \pm 0.02$ & $1.363 \pm 0.008$ & $21.40 \pm 0.17$ & $1.63 \pm 0.03$ \\
873 & 2.75 & $18.70 \pm 0.02$ & $18.76 \pm 0.02$ & $1.406 \pm 0.008$ & $26.38 \pm 0.21$ & $1.53 \pm 0.02$ \\
953 & 3.00 & $25.80 \pm 0.02$ & $25.91 \pm 0.02$ & $1.445 \pm 0.009$ & $37.44 \pm 0.34$ & $1.71 \pm 0.03$ \\
1032 & 3.25 & $40.73 \pm 0.02$ & $40.74 \pm 0.02$ & $1.534 \pm 0.010$ & $62.49 \pm 0.61$ & $1.92 \pm 0.04$ \\
1111 & 3.50 & $74.52 \pm 0.02$ & $74.59 \pm 0.02$ & $1.568 \pm 0.010$ & $116.96 \pm 1.17$ & $2.48 \pm 0.05$ \\
\hline
\end{tabular}

Table A.1: The fitted pulse heights for pad B15 in 100 Torr. The values after each of the two correction stages are shown as well. 


\begin{tabular}{ccccccc}
\hline Voltage & & $\begin{array}{c}\text { Pulse } \\
\text { Height } \\
{[\mathrm{V}]}\end{array}$ & $\begin{array}{c}\text { Drift-over-run } \\
\text { Correction } \\
{[\mathrm{mV}]}\end{array}$ & $\begin{array}{c}\text { Correction } \\
\text { Factor }\end{array}$ & $\begin{array}{c}\text { Shifted } \\
\text { Pulse } \\
\text { Height[mV] }\end{array}$ & $\begin{array}{c}\text { Shifted } \\
\text { Pulse } \\
\text { Width[mV] }\end{array}$ \\
\hline \hline 254 & 0.80 & $14.63 \pm 0.02$ & $14.77 \pm 0.02$ & 1 & $14.77 \pm 0.02$ & $1.08 \pm 0.01$ \\
330 & 1.04 & $14.33 \pm 0.02$ & $14.61 \pm 0.02$ & $1.033 \pm 0.005$ & $15.09 \pm 0.08$ & $1.12 \pm 0.01$ \\
406 & 1.28 & $13.79 \pm 0.02$ & $13.99 \pm 0.02$ & $1.074 \pm 0.005$ & $15.02 \pm 0.08$ & $1.19 \pm 0.02$ \\
483 & 1.52 & $13.30 \pm 0.02$ & $13.54 \pm 0.02$ & $1.122 \pm 0.006$ & $15.19 \pm 0.09$ & $1.29 \pm 0.02$ \\
559 & 1.76 & $12.81 \pm 0.02$ & $12.96 \pm 0.02$ & $1.183 \pm 0.006$ & $15.33 \pm 0.10$ & $1.33 \pm 0.02$ \\
635 & 2.00 & $12.47 \pm 0.02$ & $12.66 \pm 0.02$ & $1.236 \pm 0.007$ & $15.65 \pm 0.11$ & $1.42 \pm 0.02$ \\
714 & 2.25 & $12.47 \pm 0.02$ & $12.59 \pm 0.02$ & $1.284 \pm 0.007$ & $16.16 \pm 0.12$ & $1.46 \pm 0.02$ \\
794 & 2.50 & $13.25 \pm 0.02$ & $13.46 \pm 0.02$ & $1.348 \pm 0.009$ & $18.14 \pm 0.16$ & $1.52 \pm 0.03$ \\
873 & 2.75 & $15.29 \pm 0.02$ & $15.43 \pm 0.02$ & $1.397 \pm 0.008$ & $21.56 \pm 0.18$ & $1.53 \pm 0.03$ \\
953 & 3.00 & $20.17 \pm 0.02$ & $20.32 \pm 0.02$ & $1.456 \pm 0.009$ & $29.58 \pm 0.26$ & $1.55 \pm 0.03$ \\
1032 & 3.25 & $30.39 \pm 0.02$ & $30.48 \pm 0.02$ & $1.486 \pm 0.009$ & $45.30 \pm 0.43$ & $1.68 \pm 0.03$ \\
1111 & 3.50 & $51.67 \pm 0.02$ & $51.61 \pm 0.02$ & $1.518 \pm 0.010$ & $78.35 \pm 0.76$ & $1.81 \pm 0.03$ \\
\hline
\end{tabular}

Table A.2: The fitted pulse heights for pad B22 in 100 Torr. The values after each of the two correction stages are shown as well. 


\begin{tabular}{ccccccc}
\hline Voltage & & $\begin{array}{c}\text { Pulse } \\
\text { Height } \\
{[\mathrm{mV}]}\end{array}$ & $\begin{array}{c}\text { Drift-over-run } \\
\text { Correction } \\
{[\mathrm{mV}]}\end{array}$ & $\begin{array}{c}\text { Correction } \\
\text { Factor }\end{array}$ & $\begin{array}{c}\text { Shifted } \\
\text { Pulse }\end{array}$ & $\begin{array}{c}\text { Shifted } \\
\text { Pulse }\end{array}$ \\
\hline 254 & 0.80 & $35.72 \pm 0.02$ & $36.54 \pm 0.02$ & 1 & $36.54 \pm 0.02$ & $1.19 \pm 0.01$ \\
330 & 1.04 & $32.73 \pm 0.02$ & $33.84 \pm 0.02$ & $1.082 \pm 0.002$ & $36.62 \pm 0.09$ & $1.31 \pm 0.02$ \\
406 & 1.28 & $27.52 \pm 0.02$ & $28.39 \pm 0.02$ & $1.310 \pm 0.003$ & $37.19 \pm 0.12$ & $1.53 \pm 0.02$ \\
483 & 1.52 & $23.73 \pm 0.02$ & $24.38 \pm 0.02$ & $1.531 \pm 0.004$ & $37.32 \pm 0.15$ & $1.68 \pm 0.03$ \\
559 & 1.76 & $22.55 \pm 0.02$ & $23.21 \pm 0.02$ & $1.652 \pm 0.004$ & $38.34 \pm 0.17$ & $1.84 \pm 0.03$ \\
635 & 2.00 & $21.58 \pm 0.02$ & $22.10 \pm 0.02$ & $1.834 \pm 0.005$ & $40.53 \pm 0.22$ & $2.10 \pm 0.04$ \\
714 & 2.25 & $22.92 \pm 0.02$ & $23.38 \pm 0.02$ & $1.966 \pm 0.006$ & $45.96 \pm 0.28$ & $2.14 \pm 0.05$ \\
794 & 2.50 & $27.06 \pm 0.02$ & $27.55 \pm 0.02$ & $2.090 \pm 0.007$ & $57.57 \pm 0.40$ & $2.34 \pm 0.05$ \\
873 & 2.75 & $36.66 \pm 0.02$ & $37.18 \pm 0.02$ & $2.213 \pm 0.008$ & $82.29 \pm 0.65$ & $2.58 \pm 0.07$ \\
953 & 3.00 & $57.18 \pm 0.02$ & $57.78 \pm 0.02$ & $2.357 \pm 0.009$ & $136.19 \pm 1.19$ & $3.04 \pm 0.08$ \\
1032 & 3.25 & $104.15 \pm 0.03$ & $104.76 \pm 0.02$ & $2.523 \pm 0.010$ & $264.30 \pm 2.57$ & $4.26 \pm 0.12$ \\
1111 & 3.50 & $219.80 \pm 0.10$ & $220.39 \pm 0.02$ & $2.642 \pm 0.010$ & $582.28 \pm 5.77$ & $7.82 \pm 0.23$ \\
\hline
\end{tabular}

Table A.3: The fitted pulse heights for pad B31 in 100 Torr. The values after each of the two correction stages are shown as well. 


\begin{tabular}{ccccccc}
\hline Voltage & & $\begin{array}{c}\text { Pulse } \\
\text { Height } \\
{[\mathrm{V}]}\end{array}$ & $\begin{array}{c}\text { Drift-over-run } \\
\text { Correction } \\
{[\mathrm{mV}]}\end{array}$ & $\begin{array}{c}\text { Correction } \\
\text { Factor }\end{array}$ & $\begin{array}{c}\text { Shifted } \\
\text { Pulse } \\
\text { Height }[\mathrm{mV}]\end{array}$ & $\begin{array}{c}\text { Shifted } \\
\text { Pulse }\end{array}$ \\
\hline \hline 64 & 1.00 & $13.28 \pm 0.02$ & $17.20 \pm 0.02$ & 1 & $13.61 \pm 0.02$ & $1.14 \pm 0.01$ \\
86 & 1.36 & $12.67 \pm 0.02$ & $17.20 \pm 0.02$ & $1.062 \pm 0.006$ & $13.69 \pm 0.08$ & $1.19 \pm 0.01$ \\
109 & 1.72 & $12.01 \pm 0.02$ & $17.20 \pm 0.02$ & $1.115 \pm 0.006$ & $13.73 \pm 0.09$ & $1.27 \pm 0.02$ \\
132 & 2.08 & $11.63 \pm 0.02$ & $17.20 \pm 0.02$ & $1.195 \pm 0.007$ & $14.29 \pm 0.10$ & $1.33 \pm 0.02$ \\
155 & 2.44 & $11.37 \pm 0.02$ & $17.20 \pm 0.02$ & $1.242 \pm 0.007$ & $14.38 \pm 0.11$ & $1.39 \pm 0.02$ \\
178 & 2.80 & $11.41 \pm 0.02$ & $17.20 \pm 0.02$ & $1.311 \pm 0.008$ & $15.25 \pm 0.12$ & $1.47 \pm 0.02$ \\
205 & 3.23 & $12.18 \pm 0.02$ & $17.20 \pm 0.02$ & $1.391 \pm 0.009$ & $17.26 \pm 0.16$ & $1.58 \pm 0.03$ \\
232 & 3.66 & $14.13 \pm 0.02$ & $17.20 \pm 0.02$ & $1.434 \pm 0.009$ & $20.62 \pm 0.19$ & $1.63 \pm 0.03$ \\
260 & 4.09 & $17.89 \pm 0.02$ & $17.20 \pm 0.02$ & $1.521 \pm 0.010$ & $27.61 \pm 0.28$ & $1.72 \pm 0.03$ \\
287 & 4.52 & $23.38 \pm 0.02$ & $17.20 \pm 0.02$ & $1.612 \pm 0.012$ & $38.20 \pm 0.46$ & $1.84 \pm 0.04$ \\
314 & 4.95 & $33.73 \pm 0.02$ & $17.20 \pm 0.02$ & $1.684 \pm 0.012$ & $57.40 \pm 0.68$ & $2.06 \pm 0.04$ \\
342 & 5.38 & $50.42 \pm 0.02$ & $17.20 \pm 0.02$ & $1.728 \pm 0.012$ & $87.95 \pm 1.18$ & $2.03 \pm 0.05$ \\
\hline
\end{tabular}

Table A.4: The fitted pulse heights for pad B15 in 20 Torr. The values after each of the two correction stages are shown as well. 


\begin{tabular}{|c|c|c|c|c|c|c|}
\hline Voltage & $\mathrm{E} / \mathrm{p}$ & $\begin{array}{c}\text { Pulse } \\
\text { Height } \\
{[\mathrm{mV}]}\end{array}$ & $\begin{array}{c}\text { Drift-over-run } \\
\text { Correction } \\
{[\mathrm{mV}]}\end{array}$ & $\begin{array}{c}\text { Correction } \\
\text { Factor }\end{array}$ & $\begin{array}{c}\text { Shifted } \\
\text { Pulse } \\
\text { Height }[\mathrm{mV}]\end{array}$ & $\begin{array}{c}\text { Shifted } \\
\text { Pulse } \\
\text { Width }[\mathrm{mV}]\end{array}$ \\
\hline 64 & 1.00 & $6.99 \pm 0.02$ & $7.08 \pm 0.02$ & 1 & $7.08 \pm 0.02$ & $1.12 \pm 0.01$ \\
\hline 86 & 1.36 & $6.85 \pm 0.02$ & $7.03 \pm 0.02$ & $1.028 \pm$ & $7.23 \pm 0.08$ & $1.15 \pm 0.02$ \\
\hline 109 & 1.72 & $6.77 \pm 0.02$ & $6.93 \pm 0.02$ & $1.042 \pm$ & $7.22 \pm 0.08$ & $1.16 \pm 0.02$ \\
\hline 132 & 2.08 & $6.62 \pm 0.02$ & $6.71 \pm 0.02$ & $1.069 \pm$ & $7.17 \pm 0.09$ & $1.19 \pm 0.02$ \\
\hline 155 & 2.44 & $6.62 \pm 0.02$ & $6.72 \pm 0.02$ & $1.123 \pm$ & $7.55 \pm 0.10$ & $1.23 \pm 0.02$ \\
\hline 178 & 2.80 & $6.87 \pm 0.02$ & $7.03 \pm 0.02$ & $1.164 \pm$ & $8.18 \pm 0.11$ & $1.31 \pm 0.02$ \\
\hline 205 & 3.23 & $7.67 \pm 0.02$ & $7.83 \pm 0.02$ & $1.224 \pm$ & $9.58 \pm 0.15$ & $1.38 \pm 0.03$ \\
\hline 232 & 3.66 & $9.33 \pm 0.02$ & $9.46 \pm 0.02$ & $1.237 \pm$ & $11.70 \pm 0.17$ & $1.34 \pm 0.03$ \\
\hline 260 & 4.09 & $12.22 \pm 0.02$ & $12.35 \pm 0.02$ & $1.290 \pm$ & $15.93 \pm 0.25$ & $1.43 \pm 0.03$ \\
\hline 287 & 4.52 & $17.06 \pm 0.02$ & $17.22 \pm 0.02$ & $1.335 \pm$ & $22.99 \pm 0.38$ & $1.54 \pm 0.03$ \\
\hline 314 & 4.95 & $25.57 \pm 0.02$ & $25.83 \pm 0.02$ & $1.378 \pm$ & $35.60 \pm 0.61$ & $1.64 \pm 0.04$ \\
\hline 342 & 5.38 & $40.66 \pm 0.02$ & $41.08 \pm 0.02$ & $1.458 \pm$ & $59.90 \pm 1.16$ & $1.85 \pm 0.04$ \\
\hline
\end{tabular}

Table A.5: The fitted pulse heights for pad B31 in 20 Torr. The values after each of the two correction stages are shown as well.

\begin{tabular}{ccccc}
\hline Position & $\begin{array}{c}\text { Pulse } \\
\text { Height }[\mathrm{mV}]\end{array}$ & $\begin{array}{c}\text { Pulse } \\
\text { Width }[\mathrm{mV}]\end{array}$ & $\begin{array}{c}\text { Drift-over-run } \\
\text { Correction }[\mathrm{mV}]\end{array}$ & $\begin{array}{c}\text { Corrected Pulse } \\
\text { Width }[\mathrm{mV}]\end{array}$ \\
\hline 1 & $252.1 \pm 0.3$ & $20.95 \pm 0.20$ & $220.5 \pm 0.2$ & $12.42 \pm 0.13$ \\
2 & $487.9 \pm 0.5$ & $33.07 \pm 0.33$ & $455.4 \pm 0.3$ & $24.23 \pm 0.26$ \\
3 & $707.1 \pm 0.6$ & $40.49 \pm 0.41$ & $684.1 \pm 0.5$ & $34.77 \pm 0.38$ \\
4 & $842.5 \pm 0.6$ & $45.78 \pm 0.46$ & $812.3 \pm 0.6$ & $39.84 \pm 0.42$ \\
5 & $903.7 \pm 0.6$ & $43.87 \pm 0.46$ & $875.9 \pm 0.6$ & $38.76 \pm 0.42$ \\
\hline
\end{tabular}

Table A.6: Pulse heights at different locations on pad C9 


\begin{tabular}{ccccc}
\hline $\begin{array}{c}\text { Voltage } \\
{[\mathrm{V}]}\end{array}$ & E/p & $\begin{array}{c}\text { Pulse } \\
\text { Height }[\mathrm{mV}]\end{array}$ & $\begin{array}{c}\text { Drift-over-run } \\
\text { Correction }[\mathrm{mV}]\end{array}$ & $\begin{array}{c}\text { Pulse } \\
\text { Width }[\mathrm{mV}]\end{array}$ \\
\hline \hline High & Intensity & & & \\
\hline 64 & 1 & $3.43 \pm 0.09$ & $3.57 \pm 0.10$ & $1.93 \pm 0.03$ \\
127 & 2 & $4.23 \pm 0.10$ & $4.17 \pm 0.09$ & $1.92 \pm 0.09$ \\
191 & 3 & $4.90 \pm 0.10$ & $4.90 \pm 0.10$ & $2.15 \pm 0.09$ \\
254 & 4 & $5.85 \pm 0.09$ & $5.88 \pm 0.09$ & $1.92 \pm 0.07$ \\
318 & 5 & $7.57 \pm 0.06$ & $7.60 \pm 0.06$ & $1.28 \pm 0.04$ \\
381 & 6 & $17.26 \pm 0.06$ & $17.54 \pm 0.05$ & $1.21 \pm 0.04$ \\
445 & 7 & $54.96 \pm 0.07$ & $54.73 \pm 0.07$ & $1.48 \pm 0.05$ \\
508 & 8 & $197.2 \pm 0.2$ & $198.0 \pm 0.2$ & $4.1 \pm 0.1$ \\
572 & 9 & $799.0 \pm 0.7$ & $802.4 \pm 0.7$ & $15.3 \pm 0.50$ \\
635 & 10 & $3285 \pm 3$ & $3307 \pm 3$ & $58 \pm 2$ \\
\hline Low & Intensity & & & \\
\hline 572 & 9 & $25.72 \pm 0.11$ & $25.38 \pm 0.11$ & $2.40 \pm 0.08$ \\
635 & 10 & $107.8 \pm 0.4$ & $110.3 \pm 0.4$ & $9.35 \pm 0.29$ \\
699 & 11 & $500.0 \pm 1.2$ & $494.8 \pm 1.9$ & $39.9 \pm 1.4$ \\
762 & 12 & $2465 \pm 9$ & $2417 \pm 9$ & $190 \pm 6$ \\
\hline
\end{tabular}

Table A.7: The fitted pulse heights for pad B14 in 20 Torr from the maximum gain test. 